Portland State University

PDXScholar

1996

\title{
Validity, Reliability, and Utility of the Oregon Assessment for 3-5 Year Olds in Developmentally Appropriate Classrooms
}

Steffen Saifer

Portland State University

Follow this and additional works at: https://pdxscholar.library.pdx.edu/open_access_etds Let us know how access to this document benefits you.

Recommended Citation

Saifer, Steffen, "Validity, Reliability, and Utility of the Oregon Assessment for 3-5 Year Olds in Developmentally Appropriate Classrooms" (1996). Dissertations and Theses. Paper 1266. https://doi.org/10.15760/etd.1265

This Dissertation is brought to you for free and open access. It has been accepted for inclusion in Dissertations and Theses by an authorized administrator of PDXScholar. Please contact us if we can make this document more accessible: pdxscholar@pdx.edu. 


\begin{abstract}
VALIDITY, REIIABILITY, AND UTILITY OE THE OREGON ASSESSMENT EOR 3-5 YEAR OLDS IN DEVELOPMENTALIY APPROPRIATE CLASSROOMS
\end{abstract}

by

STEFEEN SAIEER

A dissertation submitted in partial fulfillment of the requirements for the degree of

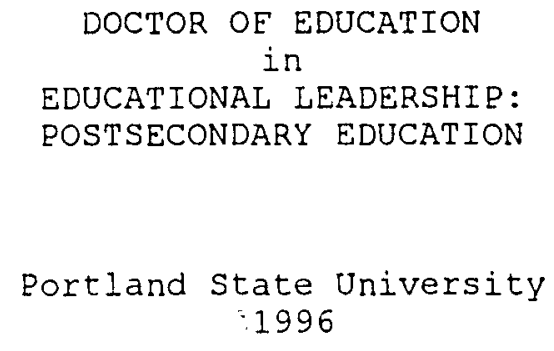


UMI Number: 9627254

\section{Copyright 1996 by} Saifer, Steffen L.

All rights reserved.

UMI Microform 9627254

Copyright 1996, by UMI Company. All rights reserved.

This microform edition is protected against unauthorized copying under Title 17, United States Code.

\section{UMI \\ 300 North Zeeb Road \\ Ann Arbor, MI 48103}


DISSERTATION APPROVAL

The abstract and dissertation of steffen Saifer for the Doctor of Education in Educational Leadership: Postsecondary Education were presented August 16, 1995, and accepted by the dissertation committee and the doctoral program.

COMMTTTEE APPROVALS:
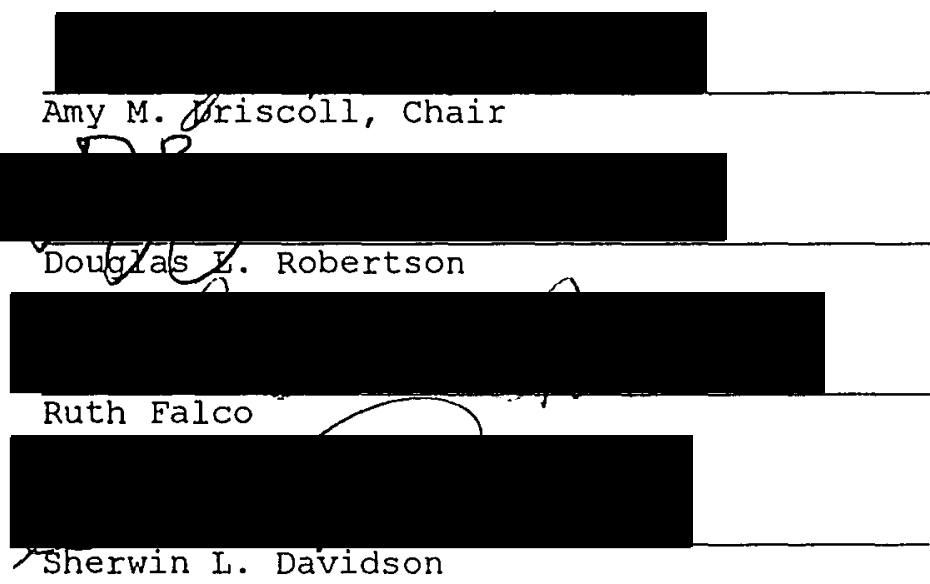

DOCTORAL PROGRAM APPROVAL:

Robert B. Everhart, Dean

School of Education

ACCEPTED FOR PORTLAND STATE UNIVERSITY BY THE LIBRARY

by

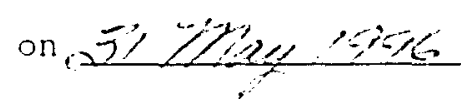


ABSTRACT

An abstract of the dissertation of Steffen saifer for the Doctor of Education in Educational Leadership: Postsecondary Education presented August 16, 1995.

Title: Validity, Reliability, and Utility of The Oregon Assessment for 3-5 Year Olds in Developmentally Appropriate Classrooms

An early childhood assessinent tool was developed by the author to fill a need for an observational, authentic, child assessment instrument that would include the measurement of social and emotional development, help guide teachers towards best curriculum practices, and meet common criteria for effective, appropriate, and equitable assessment. Theoretical constructs of interactionalism, multiple intelligences, dispositions, and developmentally appropriate practices were used to inform the construction of the tool. In addition, the author hoped to develop a tool that would be effective for use as a Head Start program evaluation research instrument. Research methods involved a representative random sample of 200 children in four early childhood programs in two northwest states and the use of two different surveys, one completed by 15 early childhood experts and one by 114 users of the assessment. 
The oregon Assessment was found to be an instrument with an acceptable degree of item, curricula, and construct validity; a high level of test-retest, inter-rater, and internal reliability (coefficient alpha $=.879)$; and an acceptable degree utility. Only one item out of 60 vas found to have low item validity, and three other items were rated lower than others (although generally positive) on both item and curricula validity. It was found to have significant but moderate congruent validity with total scores on The McCarthy Scales for Children and The Vineland Adaptive Behavior Scales. Users throughout the U.S. found it generally useful -- nearly 87 of respondents agreed or strongly agreed that the results accurately reflect the skills, behaviors, and abilities of their children. The length of time it takes to administer emerged as a concern from some users.

Recommendations were made to refine the instrument and user manual and to develop a shorter version. It was found to have great potential use as a research instrument for program evaluation and for promoting best practices in early childhood programs. 


\section{DEDICATION}

This dissertation is dedicated to my father, Abraham Saifer, for his loving and enthusiastic support and for being such a wonderful role model: he was even older than I am now when he got his doctorate. 


\section{ACKNOWLEDGMENTS}

I greatly appreciate the Head start Research Eellows grant I received. It made it possible for me to do my residency year while staying sane and allowing me and my family to eat. It also made it possible to conduct a very satisfying research project of significant scope and depth and provided a forum for national exposure for the results.

I would like to thank Amy Driscoll who talked me into to this whole thing and with great support and creativity helped me get through it. I also appreciate everyone at the Early Childhood Training Center especially Cari Olmsted, Mary Foltz, Johnnie Cain, Sally Skelding, Olga Talley, susan Hayes (you're ne:t, and Melissa Endicott -- you all have been incredibly supportive, especially during the low point, the comps.

I would like to thank the numerous PSU instructors who made the course work a wonderful intellectual challenge and growth experience for me, especially Joel Arick, Bob Everhart, Bill Greenfield, Amy Driscoll, Devorah Lieberman, and Doug Robertson. Doug was particularly inspiring in his dedication, level of knowledge, skill at teaching, and caring. I hope one day to be as good a teacher.

The program facilitated my new-found friendship with Rick. Dills and if I got through the program intact (the jury is still out on this) it was because of this friendship. Thank you Rick, it has been a highlight of the last five years. 
All the members of my immediate and extended family were great allies and supporters. I hope, Jonah, the damage caused by parental neglect will be not too severe and I thank you so much for putting up with:me. To Debi Read, my partner and greatest support of all, I am at a total loss for words to adequately express my appreciation. I hope I can return the favor with half as much grace. Thank you for being there every inch and breath of the way. 
TABLE OE CONTENTS

PAGE

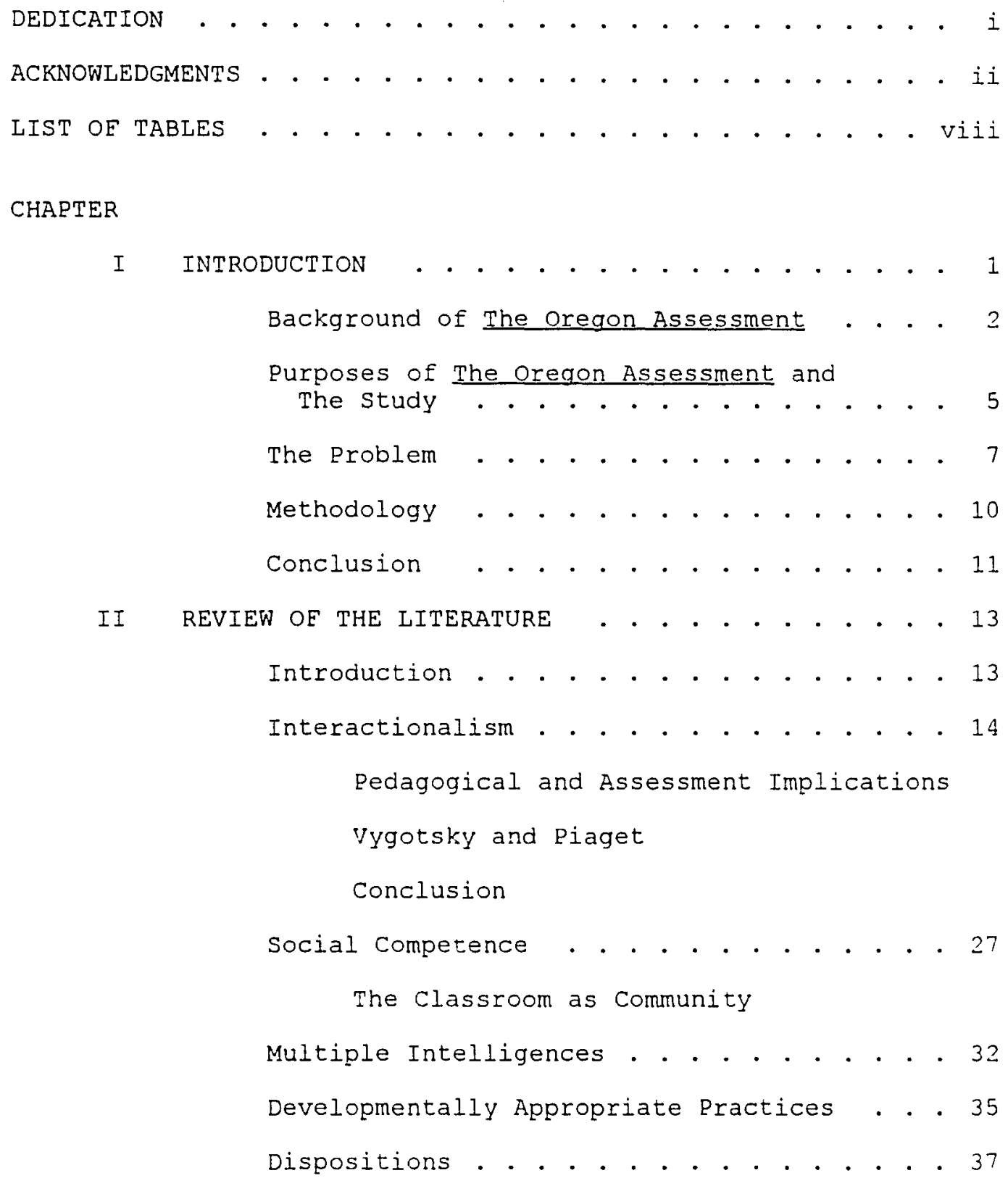


Appropriate and Effective Assessment . . . 38

Context and Authenticity

Developmental Range

Multiple Sources and Parent Input

Specificity of Use

Curriculum Planning

Use of the Child's Native Language

Equitable Assessment . . . . . . . . . 4ó

Relationality

Towards an Equitable Assessment Tool

Head Start Assessment . . . . . . . 53

Social Competence as an Educational Goal for Children from Low-Income Eamilies

The Measurement of Social Competence for Head Start Program Evaluation

Conclusion ............ . 50 ...

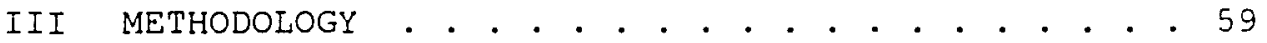

Introduction . . . . . . . . . . . . 59

Sample . . . . . . . . . . . . . 62

Procedures . . . . . . . . . . . . 65

Validity

Reliability

utility

Conclusion . . . . . . . . . . . . 70

IV

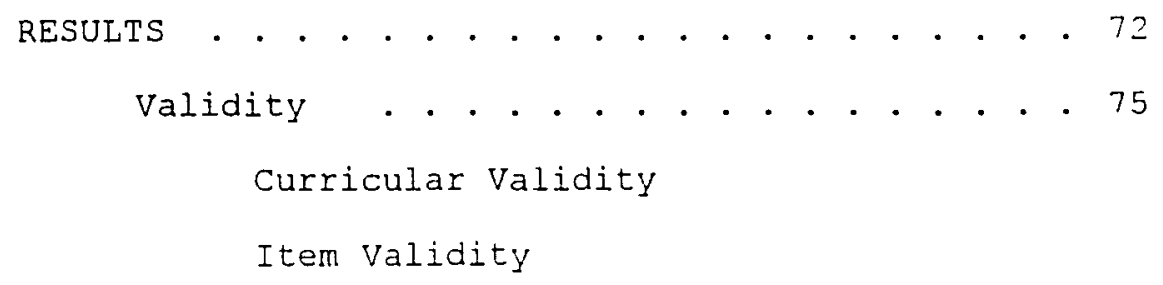


Construct Validity

Congruent validity

Conclusion

Reliability . . . . . . . . . . . . 85

Test-Retest Reliability

Inter-Observer Reliability

Internal Reliability

Utility . . . . . . . . . . . . . 90

Further Inquiry

Conclusion . . . . . . . . . . . 101

V DISCUSSION AND RECOMMENDATIONS . . . . . . 104

Curricular Validity . . . . . . . . 104

Item validity . . . . . . . . . 107

Construct Validity . . . . . . . . 108

Comments

Portfolio Assessment

Alternative Constructs

Congruent Validity . . . . . . . . . 116

Reliability . . . . . . . . . . . 118

Test-Retest and Inter-Observer Reliability

Internal Reliability

Utility . . . . . . . . . . . . . 124

Is It Helpful With Plannirg and Tracking?

Does It Provide a Rich, In-depth "Picture" of a Child?

Is It More Useful Than Other Tools? 
Other Perspectives on the Accuracy vs. Time Dilemma

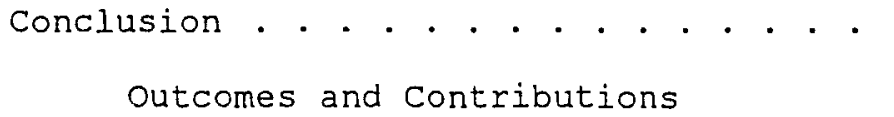

Outcomes and Contributions

\section{REEERENCES}

APPENDICES

A THE OREGON ASSESSMENT FOR 3-5 YEAR OLDS IN DEVELOPMENTALIY APRROPRIATE CLASSROOMS • • . . 149

B INDIVIDUALIZED PLANNING EORMS . . • . . . . . . . 159

C THE OREgON ASSESSMENT USER MANUAL . . . . . . . 163

D RUSSIAN AND SPANISH VERSIONS . . . . . . . . . . 199

E VALIDITY SURVEY . . . . . . . . . . . . . . . 213

E UTILITY SURVEY • . . . . . . . . . . . . . . . 220

G THE OREGON ASSESSMENT EOR 3-5 YEAR OLDS IN DEVELOPMENTALLY APPROPRIATE CLASSROOMS: SHORT VERSION . . . . . . . . . . . . . . . 224

H "ONE PAGE" EORMATS FOR THE EULL VERSION AND SHORT VERSION OE THE ASSESSMENT . . . . . . . . . . . 233 
LIST OE TABLES

TABLE

1. Experts' Ratings (Based on a 1-4 Likert-scale) of Curricular Validity for the Ten Domains and All Domains Taken Together $(\underline{\mathrm{N}}=15)$. . . . . . 71

2. Correlations Between Selected Subtests and Total Scores Between The Oregon Assessment and The McCarthy Scales and The Vineland Adaptive Behavior Scales . . . . . . . . . . . 84

3. Test-Retest Reliability and Inter-observer Reliability for oregon Assessment Subtest Scores and Total Scores $(\underline{N}=79)$. . . . . . 88

4. Internal Reliability: Correlations for Item Validity for Oregon Assessment Subtest Scores, Chronbach's Alpha, The Split-Half Correlation, and The Spearman-Brown Coefficient ( $\underline{N}=199)$. 90

5. Percentage Ratings of the Utility of The Oregon Assessment $(\underline{N}=114)$. . . . . . . 94

6. Relationships Among Several Utility Variables and Several User Demographic Variables $(\underline{N}=85)$. . 101 
CHAPTER I

INTRODUCTION

This dissertation research seeks to provide the early childhood education community with a criterion-referenced, valid, reliable, useful, and developmentally appropriate child assessment instrument that will guide teachers toward best curriculum practices. The instrument, The oregon Assessment for 3-5 Year Olds in Developmentally Appropriate Classrooms (Early Childhood Training Center, 1990) responds to the need for an observational assessment tool that measures social skills, selfconcept, play behaviors, dispositions, and cognitive processing skills. As a developmentally appropriate assessment tool it guides teachers towards practices congruent with exemplary practices as defined by the National Association for the Education of Young Children (Bredekamp, 1987; Bredekamp \& Rosegrant, 1992; NAEYC, 1991). The instrument is directed to the following purposes: (a) assessing current child functioning, (b) assisting teachers in setting developmentally appropriate goals and planning an individualized curriculum, (c) tracking child progress, (d) providing a basis of dialogue with parents, and (e) providing information for referrals. In addition, if found valid, reliable and useful, it could provide the research community, particularly Head start, with a research tool for effective program evaluation. 
Background of The Oregon Assessment

The assessment in its current form was conceived by the author and developed with a team of oregon Head start Education Coordinators under his direction. Its original target audience was Head start staff, but its use is not limited to that group. It was field tested at three Head start programs for one year and revised to include feedback from teachers regarding its utility, scoring, and wording of items. It is currently in its fifth version, having been further revised in response to feedback from users.

The impetus for its development came from a need expressed by Head Start staff for a tool for teachers to assess children globally, with an equal balance of social, emotional, and cognitive indicators. This emphasis reflects the primary goals these educators have for their children -- the development of social competence and a healthy self-concept. Developing children's social competence is also the primary goal of the federal Head Start program (Raven \& Zigler, 1991; U.S. Department of Health and Human Services, 1984). The instrument includes items to assess social development, self-concept, play skills, dispositions, and cognitive skills (particularly process skills such as problem solving). The cognitive skills are oriented toward dispositions and a developmentally appropriate curriculum context lobserved during play and typical classroom routines). Rather than the more typical assessment strategy of one-to-one direct questioning by a stranger using materials unfamiliar to the child, all items are scored by observation in 
natural classroom settings. This method of authentic assessment reflects guidelines and recommendations from early childhood education professional organizations for appropriate assessment of young children (NAEYC, 1988; NAEYC, 1991; NASP, 1991; SACUS, 1990).

The full version of The Oregon Assessment consists of 60 items in ten areas of development (see Appendix A). This was the version used in this dissertation research project. It contains some unique features not found on most assessment tools. Among these features are an emphasis on social and intrapersonal development and cognitive processing, an assessment of play skills, ample space to encourage descriptive comments, and a five point rating scale for each item. The scoring is keyed to the frequency of the observed behavior as well as the degree of assistance required from the teacher for the child to perform the behavior at his or her highest level of ability.

Many traditional assessments do not adequately examine the social functioning of children or the interactivity between teacher or parent and child and thus fail to provide important information that can be used to optimize children's growth and development. The Oregon Assessment contains seven specifically social items and four of the six language items are related to interactive communication. In addition there are two play items related to social interaction and at least 13 other items in various domains that have a clear social component. The social interaction between teacher and child, an important element in 
children's educational development (Marcon, 1994; Vygotsky, 1978), is assessed by a number of items on the instrument as well during the scoring process.

Theoretical constructs considered in the construction of the tool were Vygotsky's $(1978,1981)$ theory of interactionalism, Gardner's (1983) construct of multiple intelligences, Katz's $(1990,1991)$ positive dispositions as educational goals, and principles of developmentally appropriate practices in early childhood education (Bredekamp, 1987; Bredekamp \& Rosegrant, 1992).

The Oregon Assessment is one part of a system to help teachers assess children, set goals for them, plan individualized curriculum, and track progress. Towards that end a set of forms were developed (see Appendix B). These forms are integral to the assessment in that they help teachers develop responsive and flexible curriculum practices based on the needs of the particular children they serve -- a process necessary for quality education of young children (Bredekamp, 1987; Bredekamp \& Rosegrant, 1992; Katz, 1990, 1991; Saifer, 1990; U.S. Department of Health and Human Services, 1984).

In addition, a user manual was developed to help teachers use the instrument effectively by providing information about its purpose, clarification of the meaning of items, and assistance in scoring. It also served an important purpose in this dissertation research by helping to increase inter-observer reliability. The User Manual for the 60 item (full) version of the Assessment can be found in Appendix $C$. 
The assessment is in use in many early childhood programs throughout the:U.S. and Europe, although most heavily used in the Northwest. It has been translated into Russian, Spanish, Hungarian, and a number of other languages. Appendix D includes copies of the Spanish and Russian versions.

\section{Purposes of The Oregon Assessment and The Study}

A very important purpose of The Oregon Assessment is to promote socially oriented, developmentally appropriate curricula practices. An axiom in education is that "assessment drives curriculum" (Bricker \& Iittman, 1982; Shepard, 1991; Wiggins, 1989), Teaching to the test is a well documented and pervasive concern in education, which can result in inappropriate curricula practices damaging to children (Hill, 1987; NAEYC, 1988; Shepard, 1991; Shepard \& Smith, 1986; Wiggins, 1989). An assessment that has been developed from an examination of best practices and from educational goals as defined by educators, rather than from a list of decontextualized, cognitively oriented skills, should have a positive impact on curricula. Teachers teach to the test for good reason: they desire positive outcomes for their children and want measurements to show that they are effective educators. It is unlikely that teachers, on a large scale, can be convinced not to teach to the test as long as tests carry so much weight in U.S. culture and test scores are used to measure teacher and program effectiveness (Hanson, 1993; Meisels; 1987). A more realistic approach is to change the rature of the tests in use so that "teaching to the test" 
will mean utilizing appropriate practices and will promote the development of children's social abilities, intrapersonal competence, positive dispositions, and cognitive processing skills (Wiggins, 1989).

The inevitable link between assessment and curricula can be desirable when it leads to positive outcomes (Bagnato \& Neisworth, 1981; Bricker \& Littman, 1982). The Oregon Assessment is designed to intentionally make this link for teachers through the wording of items, the nature of the items selected, the nature of the scoring system and the individualized planning forms that help teachers use assessment information to write goals and then plan activities (Appendix B) Utilizing The oregon Assessment, with many items for measuring social competence, will help teachers and parents more effectively analyze interpersonal abilities and view them as critical to the healthy growth and development of their children. This, in turn, should lead to a greater emphasis on this domain in the curriculum.

Early childhood educators have decried the lack of assessment tools that meet the criteria for appropriate, effective and equitable assessment, that include social functioning and a broad range of developmental skills, and that are eminently useful to teachers (California state Department of Education, 1988; Gammage, 1991; Kamii, 1990; Meade-Roberts, 1988; Meisels, 1987; NAEYC, 1983; NASBE, 1991; NASP, 1991; Raven \& Zigler, 1991; SACUS, 1990; Walton \& Nuttal1, 1992; WexlerSherman, Gardner, \& Feldman, 1988). If The Oreqon Assessment 
can meet these conditions and also prove to be a valid and reliable tool, then it will fill a great need. In addition, it could be used for research purposes to ascertain program effectiveness through a pre/post-test design. As The oregon Assessment is already widely used in many Head Start and early childhood programs (and it can serve a particular, useful function in any early childhood program), its use as a research tool to ascertain program effectiveness would be a relatively simple process, requiring no additional testing of children or extensive staff training.

The Problem

This research project sought to determine if the oregon Assessment is a valid, reliable, and useful instrument so that it can best fulfill its intended purposes. Towards that end the research attempted to answer the following questions:

\section{General Research Question}

Is The Oregon Assessment for $3-5$ Year olds in

Developmentally Appropriateclassrooms a valid, reliable, and useful assessment tool?

\section{Specific Research Questions}

Research Question 1: Is The Oregon Assessment a valid tool to assess the functioning of young children three to siz years of age in preschool classrooms?

Research Question 1a: Does The Oregon Assessment have curricular validity -- does it match the objectives 
of developmentally appropriate curriculum practices? Research Question 1b: Does The Oregon Assessment have item validity -- is each item an important indicator of the domain it is under?

Research Question 1c: Does The Oregon Assessment have construct validity -- does the test reflect the guidelines for appropriate and effective assessment of young children? Research Question 1d: Does The Oregon Assessment have congruent validity -- do children score similarly on The Oregon Assessment as on other, more established, well-researched instruments that purport to measure similar areas of functioning?

Research Question 2: Is The Oregon Assessment a reliable tool to assess the functioning of young children three to siz years of age in preschool classrooms?

Research Question 2a: Does The Oregon Assessment have test-retest reliability?

Research Question 2b: Does The oregon Assessment have inter-observer reliability?

Research Question 2c: Does The oregon Assessment have internal reliability?

Research Question 3: Is The oregon Assessment a useful tool for preschool teachers to effectively assess and plan for children?

Research Question 3a: Do teachers and administrators perceive The oregon Assessment to be accurate? 
Research Question 3b: Do teachers and administrators perceive that The Oregon Assessment provides them with important information about children?

Research Question 3c: Do teachers and administrators perceive The Oregon Assessment User Manual to be clear and understandable? Research Question 3d: Do teachers and administrators perceive that The Oregon Assessment is effective in helping to write goals? Research Question 3e: Do teachers and administrators perceive that The Oregon Assessment is effective for curriculum planning and tracking children's progress? Research Question 3f: Do teachers and administrators perceive that The Oregon Assessment is more useful than other assessment tools that they have used? Research Question 3g: Do teachers and administrators perceive that parents respond favorably to the oregon Assessment?

Research Question 3h: Do teachers and administrators perceive that The oregon Assessment is useful for helping parents understand their children and set appropriate goals for them?

Research Question 3i: Do teachers and administrators perceive that The oregon Assessment is responsive to diversity? Research Question 3j: Do teachers and administrators perceive that the completed assessment provides a 
rich and in-depth "picture" of a child?

Research Question 3k: How long does it take to complete the assessment on each child?

Research Question 31: Over how long a period of time

do teachers administer an assessment on a child?

Research Question 3m: Do teachers and administrators

perceive that The Oregon Assessment takes a

reasonable amount of time to administer?

Research Question 3n: Do: teachers and administrators

find the scoring system to be understandable?

Methodology

The answers to the research question were sought through a multi-faceted research design. To determine curricular, item, and construct validity, a questionnaire was mailed to 30 carefully selected early childhood education experts. A representative, random sample of 200 children was used to determine congruent validity and the three types of reliability: test-retest, inter-observer, and internal. Congruent validity was determined by correlating scores on The Oregon Assessment with scores on McCarthy Scales for Children (McCarthy, 1972) and The Vineland Adaptive Behavior Scales (Classroom Edition) (Sparrow, Ball, \& Cicchetti, 1984). Test- retest reliability was determined by the same teacher administering the test twice to the same child within three weeks. Inter-observer reliability was determined by having two different people familiar with the child and trained on the assessment (typically 
a teacher and a supervisor or assistant teacher) administer the test to that child at about the same time. Internal reliability was determined statistically by obtaining Chronbach's alpha, the split-half correlation, the Spearman-Brown coefficient and item reliability for each item, domain, and the total score from all useable scores on the assessment ( $N=199)$. In addition, a 19 item survey was sent to 200 teachers and administers to determine the utility of the assessment on a variety of dimensions.

\section{Conclusion}

Potentially, the results of the research undertaken for this dissertation on the oregon Assessment can have a significant and positive impact on early childhood programs, including Head start, and on future research. It can provide a measure for child functioning specific to the theoretical construct of interactionalism and the guidelines for developmentally appropriate practices. It can assess children's progress in the areas of development reflective of teachers' actual practices and goals for children as recommended by national early childhood education leadership associations and agencies.

Use of the oregon Assessment has the potential to guide programs toward effective educational practices by positively influencing curriculum practices. The instrument contains implicit but strong messages regarding developmentally 
appropriate teaching practices by the very nature of what it measures and how it asks teachers to gather data.

The oregon Assessment has the potential to be a valid, reliable, useful, equitable, contextualized, observational assessment tool that measures a wide range of abilities and behaviors across many developmental domains, particularly social functioning. Towards this end, research was conducted to determine the validity, reliability, and utility of the oregon Assessment. 
CHAPTER II

REVIEW OE THE LITERATURE

Introduction

The following review of literature will begin with an exploration of the construct of interactionalism, as it forms the core theoretical basis for the development and design of The oregon Assessment. This is followed by a discussion of other key influences on its development and design: the importance of assessing and fostering social competence, the theory of multiple intelligences, the construct of developmentally appropriate practices, the concept of dispositions as educational goals, the need for assessment tools that adhere to the principles of appropriate, effective, and equitable assessment, and issues of Head Start assessment and evaluation. Taken together these issues, needs, and constructs are the raison d'etre of the oregon Assessment. They drove its development, as practitioners sought an assessment tool that was reflective of these constructs and responsive to the concerns raised by these issues. That the needs and concerns of practitioners drove its conception and development, provided a good start for the creation of a valid and useful tool. It was not developed in isolation from the field nor simply adapted from existing models or instruments. However, demonstration of 
the actual efficacy of The oregon Assessment and its refinement are the driving forces of this research study.

\section{Interactionalism}

Many of the unique features of The oregon Assessment stem from a theoretical construct based on the work of Lev Semenovich Vygotsky (1981) who postulated that all higher mental functions develop from early social interactions. According to Vygotsky, development cannot be abstracted from a social, historical, and cultural context. This context is reflected most clearly in the social interactions between the child and others, hence interactionalism. The Oregon Assessment measures the quality and quantity of many social interaction phenomena across a variety of domains, including child to child interactions, child to adult interactions, non-verbal communication, the ability to form and maintain friendships, and more.

Vygotsky was born in 1896 in Orsha, Belarus and died from tuberculosis at the age of 37 . In his short life he was a major intellectual figure in the (then) Soviet Union. He lived during the revolution and was influenced by the heady freedom of the time to break new intellectual ground. He was a well rounded person with a great love of poetry, literature, theater, and art. Vygotsky kept informed of trends in psychology, the arts, and philosophy throughout Europe and interpreted the writings of Piaget and others. He wrote broadly on many subjects and became interested in psychology in an attempt to understand human development and the relationship between people and society. 
The task of the Soviet Union at the time -- creating a new social order and restructuring institutions (particularly the education system) -- gave further impetus and a forum for Vygotsky to pursue a new perspective on the individual in relation to society.

He believed in a dialectical view of human development (a dynamic process defined by relationships between phenomena and their inherent contradictions) in which "All the higher functions originate as actual relations between human individuals" (Vygotsky, 1978, p. 57). Hence, Wertsch (1985) described his cognitive theories as the "social formation of mind." The interrelationship between the biological/maturational forces of development (called "natural" development by Vygotsky) and the social/cultural/ historical forces, with the later considered the more important determinant of human behavior, is primary to Vygotsky's theories.

Although best known for his essay, Thinking and Speech, Vygotsky (1987) is not strictly a cognitive theorist.

We often describe a child's development as the development of his intellectual functions; every child stands before us as a theoretician who, characterized by a higher or lower level of intellectual development, moves from one stage to another. But if we ignore the child's needs, and the incentives which are effective in getting him to act, we will never be able to understand his advance from one developmental state to the next, because every advance is connected with a marked change in motives, inclinations, and incentives. (p. 92)

Clearly, Vygotsky takes a more holistic view of development.

without social interaction many higher functions such as deliberate attention, logical memory, verbal and conceptual 
thought, and complex emotions could not emerge, according to vygotsky. Social communication from the earliest years is internalized forming the basis for these higher functions.

It is necessary that everything internal in higher forms was external, that is, for others it was what it now is for oneself. Any higher mental function necessarily goes through an external stage in its development because it is initially a social function. This is the center of the whole problem of internal and external behavior. ... When we speak of a process, "external" means "social". Any higher mental function was external because it was social at some point before becoming an internal, truly mental function. (Vygotsky, 1981, p. 86)

This emphasis on the role of society and culture (primarily mediated through language) in influencing development is the most unique attribute of vygotsky's theories and has great implications for pedagogy, the role of the teacher, and ultimately assessment practices. To influence early childhood pedagogy towards a more interactive mode with an emphasis on social functioning and attention to quality of the relationship between teacher and learner, an assessment tool with such emphases is needed. The proposed oregon Assessment is designed to help address that need. If the nature of the items and the process of scoring reflect these values, then pedagogy will be affected accordingly, as assessment tends to drive curriculum (Shepard, 1991). If only a narrow band of cognitively oriented, skill-based, and non-functional behaviors are assessed, then curriculum practices will promote the development of similar behaviors . 


\section{Pedagogical and Assessment Implications}

Vygotsky's (1978) most significant contribution to pedagogy and assessment is the concept of the zone of proximal development. This is the difference between the performance of a child left to him/herself and the performance of a child when given assistance by an adult. Two children may score at the five year old level on an item on a psychometric test, but with assistance from an adult one may score at a six year old level and the other at an eight year old level. The first child's zone of proximal development is one year and the second child's is three years. Teaching strategies which target the child at his or her zone of proximal development are the most effective, according to Vygotsky. The oregon Assessment was designed to assist teachers in doing this by asking them to consider the degree of teacher assistance a child may need to achieve the desired skill or behavior for each item. This is one of two main criteria for scoring an item, the other being the frequency of the behavior.

Teaching in the zone of proximal development does not imply that the assistance should be directive. Vygotsky (1978, pp. 88-89) deplored learning by imitation and believed in the importance of helping children to gain autonomy and to understand their thinking processes rather than teach concrete skills or behaviors. He criticized Montessori's approach to writing because it is a rote exercise in motor skills not a "complex cultural activity" (p. 118). In discussing the most 
effective ways of teaching writing to young children Vygotsky recommended that:

Writing should be incorporated into a task that is necessary and relevant for life. Only then can we be certain that it will develop not as a matter of hand and finger habits but as a really new and complex form of speech. Writing should be meaningful to children, an intrinsic need should be aroused in them. Writing should be taught naturally. The activity should be engaged in the course of children's play, and writing should be "cultivated"rather than "imposed". Natural methods of teaching reading and writing involve appropriate operations on the child's environment. Reading and writing should become necessary for her in her play (Vygotsky, 1978, p. 117-118).

Child-centered learning during play for young children is integral to Vygotsky's pedagogy. Items on The oregon Assessment are to be scored from observing children during routines or in play. For example, the math items state, "When playing with objects in the environment such as blocks, pegs, dishes, etc.: Matches (i.e.; red cars into red container)" (item \#50) or "Seriates (i.e.; lines up cars smallest to largest)" (item \#52). This exemplifies assessment that both reflects and can drive a child-centered pedagogy.

The concept of the zone of proximal development also has implications for the definition and measurement of intelligence and functioning. From an interactionalist perspective intelligence is dynamic, process oriented, and cannot be defined outside of a social context. It is more related to potential than performance. At least one measure, perhaps the primary measure, of intelligence would be the size of the zone of proximal development. This is a dynamic, rather than static measurement changing each time any member of the dyad changes. 
The process of scoring the oregon Assessment entails, in part, determining how much assistance is required for the child to demonstrate the ability or behavior, in essence measuring the zone of proximal development (for that behavior with that teacher at a given point in time).

This leads to another important concept developed by Vygotsky (Tudge, 1991) with great implications for assessment: the dyad as the smallest unit of analysis. Most assessments attempt to provide an individual measure of functioning. A Vygotskian perspective challenges the notion that this is possible. The answer to a question or the demonstration of skill is always done within a social/cultural context. This context is formed by the history, values, and meanings that both the "question asker" (what is asked and how) and the "question answerer" bring to the interaction, regardless of whether it was written down years before or asked spontaneously. The result of the interaction (the "score") is only a reflection of that particular dynamic at that particular time. It is bound by context and relationship. More accurate, meaningful, and useful information is obtained by recognizing this and making it an explicit aspect of the assessment process. The Oregon Assessment attempts to do this by asking the teacher to consider cultural meanings the child may bring and consider the teacher's own interactive role (level of helping) when scoring, as previously described.

A social view of intelligence and functioning has implications for outcomes for children. A logical extension of 
this line of reasoning leads to this outcome for children: To become productive members of a particular society and culture who enjoy the rewards of living in that society and who contribute positively to its advancement. This clearly involves an array of sophisticated skills and abilities (higher mental functioning). It is a very different outcome than one would draw from many pedagogical theories that focus primarily on cognitive functioning, the acquisition of facts, and/or obedience to authority. Eollowing the thread back to the early childhood teaching practices that will most effectively lead to this outcome, high social functioning, creativity, communication, imagination, and self-awareness become primary. These skills and abilities are included on The oregon Assessment.

\section{Vygotsky and Piaget}

It is important to discuss here the relationship between Vygotsky's (1978) interactionalist theory of development and Piaget's (Bidell, 1988) constructivist theory as the Piagetian paradigm has dominated early childhood constructs for test development and educational practice for decades. There are great areas of agreement between these two developmental psychologists (who were contemporaries) and some very fundamental theoretical differences. However, their theories complement each other in many ways, each filling in the other's gaps.

Vygotsky and Piaget both viewed development as a dialectic (Bidell, 1988). This dynamic view would lead both to see the 
child as an active creator of his/her own development and constructor of knowledge. They both were interested in the relations between the social and individual aspects of development, between structure and function in development, I between cognition and emotions, and between action and representation. They agreed on the concept of internalization. Vygotsky embraced Piaget's ideas on children's moral development.

However, Vygotsky departed from Piaget sharply on a number of points. He believed that development follows learning, whereas Piaget believed that learning follows development. The concept of the zone of proximal development reflects that theory. The "Piagetian dilemma" (Duckworth, 1987) -- what is the teacher's role if learning follows development? -- is resolved through the vygotskian perspective. The teacher actively promotes development by challenging children in the zone of proximal development. The teacher leads a child towards that next developmental step through a variety of interactive strategies. A thorough understanding of the developmental steps as described by Piaget is necessary in order to help the child move through them. The role of the teacher is not to move the child more quickly through these stages, but to present new information, a different perspective, help the child apply existing information she or he already has to gain a new understanding, structure the materials and environment so that information becomes more clearly understandable, provide opportunities to explore the many ways information can be applied and 
modified, expand the child's vocabulary (and therefore the number of linguistic tools), and improve the child's ability to access and process information. In short, to "...broaden and deepen their scope by opening up parts of the world that children may not, on their own, have thought of thinking about" (Duckworth, 1987, p. 49).

Piaget primarily focused on the individual's stages of cognitive development; Vygotsky on development in a social context. This was particularly true in their empirical research, less true in their theoretical writings. Theoretically they both realized the integral connection between the individual and social influences on development. This is another area where they complement each other. The concept of stages of cognitive development is a viable construct and essential to understanding children; however without social interaction, especially language, cognition will not develop. The differences between Vygotsky and Piaget are differences of emphasis. Classrooms with a Piagetian emphasis would have a greater focus on equipment and materials and the child in isolated experimentation. Classrooms with a Vygotskian emphasis would have a greater focus on verbal interactions, sociodramatic play, and the creation of a classroom community. Both, of course, would have many elements of the other, looking quite similar in that children would be active, engaged in play, and self-directed. Because it includes many more items related to positive social interaction and meaningful connections with others than most early childhood assessment instruments (which 
are typically based on Piagetian theory), The oregon Assessment

both reflects interactionalism and guides users towards

interactionalist practices.

The Role of Play in Development. In regards to play, Vygotsky (1978) believed that play is "...a leading factor in development" (p. 101) for young children. Piaget viewed play as a means by which children can assimilate information but not actually learn or develop because they do not accommodate during play (Eorman \& Kuschner, 1983). To Vygotsky, not only is play an essential activity for children but it creates development. However, by also using play as the basis upon which to teach in the zone of promixal development, development can be enhanced even more. Social play is an important aspect of the curriculum from a Piagetian perspective, but it is the most vital aspect of the curriculum, from a Vygotskian perspective. "A child's greatest achievements are possible in play, achievements that tomorrow will become her basic level of real action and morality" (Vygotsky, 1978, p. 100). Play provides a transition between the very concrete world of young children and the later ability to think purely in symbols. (However, imaginative play does not directly involve the use of symbols because the goal of the play, according to Vygotsky, is not to represent but to fulfill wishes and needs). Through play the child consciously acts and gives meanings to objects, developing the ability to think abstractly. In play the child can act independently of what she or he sees, actions derive from ideas rather than things (after about three years of age), and the child separates 
fantasy from reality. The child in play places constraints and rules on his behavior (more and more so as the child develops). In these ways, play stretches the child's capabilities and capacities and thus causes development (creating a zone of proximal development). "In play a child always behaves beyond his average age, above his daily behavior; in play it is as though he were a head taller than himself" (Vygotsky, 1978, p. 102). This provides ample justification to include play as a developmental domain on the oregon Assessment, and explains why it is rarely included as such on typical assessments: few other theories of development or pedagogy give play such a central, vital place. The inclusion of play skills, especially socially interactive play skills, on The oregon Assessment stems directly from the theoretic construct of interactionalism and the central role of play in that theory.

Eor teachers to utilize play in the zone of proximal! development requires the ability to assess each child's level of functioning and then provide a new piece of information or skill that is just above that level. Setting up compeling, challenging, and complex play environments and intervening: effectively requires a good understanding of children's needs and desires, determined by careful and objective listening and observing. The oregon Assessment is intended to help facilitate this as it contains items assessing specific play skills and numerous items related to social and cognitive functioning in a play context. 
Teachers must have the ability to use words and actions that naturally and non-intrusively follow the child's lead (so that they are meaningful and relevant to the child) to that next level. This requires spontaneity, immediacy of action, a willingness to forgo preconceived teacher-directed strategies, and a willingness to learn from children. Such skills can be encouraged through appropriate assessment and honed through specific training and much practice. In a study by Schrader (1988), teachers were trained in this approach and then they were analyzed for their ability to facilitate literacy development within the context of children's dramatic play. Each classroom contained three identically equipped dramatic play areas: house, post office, and office. There was great variance in teacher's abilities to do this effectively, however all teachers were more facilitative than directive and several teachers were able to demonstrate an impressive array of skills and actions that clearly produced growth in children's reading and writing capabilities during play.

Implied in the practices described above is the idea that teachers model active teaching and learning strategies by participating in a dynamic process. Effective education requires that learning influences flow back and forth between teacher and child. This positive teaching style -- being respectful, responsive, engaging, and democratic -- correlates highly with positive social behaviors and social problem solving abilities in children (Hollowly \& Reichhart-Erickson, 1988). It is most effectively facilitated in play. 
The ability to teach social skills formally (through books, puppets, role plays, etc.) and informally (by assisting children to solve conflicts and negotiate social situations when they arise) is also important. Negotiation skills and problem solving skills are assessed on The oregon Assessment in a number of ways. This assists teachers in helping children to move from self-speech to more social speech, utilize verbal and nonverbal social cues (eye contact, smiles, enthusiastic voice tones, etc.), effectively enter play, and diplomatically negotiate the direction of play (rejecting others' ideas while respecting them, suggesting ideas without being pushy) (Hazen, Black, \& Eleming-Johnson, 1984). There is a complex array of skills and dispositions needed to play effectively and most teachers would benefit in facilitating those skills and dispositions in children from the indirect guidance provided by The Oregon Assessment.

\section{Conclusion}

Vygotsky has devised a theory of human growth in which social interaction plays the significant role in driving development. All higher functions derive from/this. Social interaction and playful adult/child interchanges lie at the heart of fostering development in early childhood. By assessing the zone of proximal development and providing learning opportunities within it, development is further enhanced, broadened, and deepened. By making social outcomes a priority and creating classroom environments highly conducive to social interactions through play -- facilitated by a focus on social 
skills in the assessment process -- early childhood teachers can have a positive impact on the lives of children.

\section{Social Competence}

Eostering social competence is important for developmental, psychological, and educational reasons. Children with inadequate peer relations are at risk for mental health, social, and emotional problems later in life (Cowen, Pederson, Babigian, Izzo, \& Trost, 1973; Kohlberg, Lacrosse, \& Ricks, 1972). There is some research evidence that when teachers focus on social competence they view students more favorably (improving student-teacher relationships), alter instructional strategies, and increase expectations which leads to improved student performance (Carrasco, 1979). Improvements in social skills are clearly linked to gains in I.Q. (Saltz, Dixon, \& Johnson, 1977), problem solving abilities (Simon \& Smith, 1983), language development (Smith \& Syddall, 1978), and fewer acting out behaviors (Glanville \& Sundberg, 1987). Kindergarten children who engage in a great deal of socio-dramatic play (the most social and interactional form of play) have higher levels of reading skills, word-writing fluency, and a variety of language skills than children who engage in little sociodramatic play (a better predictor than sex or socioeconomic class) (Pellegrini, 1980). The value of these findings is starting to be recognized by educators. Emphasis on the social nature of learning is one of five key components of quality primary education as 
identified by educators in the provinces of Alberta and British Columbia, Canada (Gammage, 1991). The Southern Association on Children Under Six (SACUS) identified "learning how to live comfortably with others" as one of eight needs of children that must be addressed for quality child care (SACUS, 1990). However, social compeience continues to be undervalued in assessment processes. This gives an implicit but strong message to teachers and parents that it is ultimately not very important.

Viewing pedagogy and child development from an interactionalist perspective begs the question: Are social outcomes worth pursuing in an educational setting? As previously discussed, there is ample evidence that when children improve their social skills they also make gains in a variety of cognitive areas. However, an argument can also be made that social outcomes themselves are highly valuable regardless of the correlation between social acuity and cognitively oriented outcomes. Vygotskian theory implies that social outcomes are intrinsically valuable, as social interaction is the basic construct from which all other higher development derives. The ability to effectively engage with others in meaningful social interactions promotes the development of both individuals, particularly the individual functioning at a lower developmental level. The teacher who can communicate clearly and succinctly, who can adapt her interactions to meet the individual needs and communication styles of her pupils, and who has the ability to foster positive communication in the classroom, would be highly 
effective from a Vygotskian perspective. This construct, intersubjectivity (the connection between individuals through mutual, shared meanings), has clear implications for test development and subsequent pedagogy driven by tests (Wertsch, 1991). The many items on The oregon Assessment related to communication and positive social functioning, especially "Listens to and contributes to group discussions at mealtimes \& sharing times" (item \#28) and "Initiates and sustains conversations" (item \#33), respond to those implications. Lasch fears that the lack of ability or availability for people to engage in social discourse has endangered the fabric of American society.

Let us begin with a simple proposition: What democracy requires is public debate, not information. of course it needs information too, but the kind of information it needs can be generated only by vigorous popular debate. We do not know what we need to know until we ask the right questions, and we can identify the right questions only by subjecting our own ideas about the world to the test of public controversy. Information, usually seen as the precondition of debate, is better understood as its by-product. When we get into arguments that focus and fully engage our attention, we become avid seekers of relevant information. Otherwise, we take in information passively - if we take it in at all. (Lasch, 1991, p.72)

Lasch contends here that social interaction drives knowledge, an idea congruent with Vygotsky's theory. If one major role of the education system in America is to prepare children to become active participants in the democratic process (Dewey \& Tufts, 1932), than a social basis for education practices may be the most expedient means towards that end. 
It can also be argued that social skills are as important and perhaps more important than cognitive skills or specific abilities for success in practical areas in life. Almost everyone knows a person with great ability and high cognitive intelligence who has failed on the job or has great difficulty in his or her personal life because of poor social skills. Conversely, many people experience great personal and professional success in spite of limited cognitive capacity or । little mastery of academic subject matter due to excellent social skills (Clark, 1990; Dewey \& Tufts, 1932; Kegan, 1982; Schutz, 1966; Vaillant, 1977).

\section{The Classroom as Community}

creating a close-knit social network in the classroom is a powerful way to promote and develop social competence in children. Paley (1990) describes an educational approach involving storytelling in order to create such a community in her classroom. Children choose other children to act out roles in the stories they create. Through this method, which has social outcomes as its major goal, a child who socially isolates himself and has some unusual and disturbing behaviors (repetitive statements and actions, unresponsiveness to others) : becomes part of the group and becomes much more coherent and responsive. Paley provides strong evidence that a focus on social interactions can result in great improvements in the functioning of a disturbed child. Remediative and therapeutic results can be realized in the short term when the long term 
goals are social outcomes. In describing her interactive storytelling and dramatization process, Paley stated:

Here is an intuitive and spontaneous set of responses from which teachers and children can create the rules and traditions that govern listening and responding. In the telling and performing of stories, all ideas must be heard, considered, compared, interpreted, and acted upon. The bridges built in play are lengthened, their partially exposed signposts organized and labeled in ways that commit the storyteller to travel in particular directions. The subject encompasses all| of language and thought: It is the academic inheritor of the creative wisdom of play. (p. 35)

Here Paley describes a language rich, interactive, social process, facilitated and mediated by the teacher. She separates it from play and refers to it as academic, however it can also be viewed as a type of play, thematic-fantasy play that is created by the children themselves (Johnson, Christie, \& Yawkey, 1987, p. 248). It is social play during which an adult can extend children's social and cognitive learning in their zones of prozimal development. The beneficial results of this method are clearly documented by Paley in the progress of the child described previously. ISocial outcomes are valid onto themselves, not just for the results they may have on other areas of development. Both'short term and long term effects can be highly positive for children. Teachers would do well to create a social focus for their classrooms.

The Oregon Assessment, with 27 items out of a total of 60 items clearly assessing interpersonal abilities, is heavily weighted towards measuring social competence. The remaining 33 items include a number with social elements. These include, "Listens to entire story during storytime" in the cognitive- 
Literacy domain (item \#55) which implies child is not disruptive of others, and "Displays resourcefulness when faced with problems or obstacles - can generate several solutions/alternatives when asked questions such as 'What else can you do..'" (item \#49) in the Cognitive-Problem Solving domain which can be measured when problems or obstacles involve conflicts with other children.

\section{Multiple Intelligences}

Like interactionalism, constructivism, and the importance of the social domain, Gardner's (1983) theoretical construct of multiple intelligences contributed significantly to the development and formation of the oregon Assessment. Gardner postulated that while traditional education focuses primarily on only two types of intelligence, linguistic and logicalmathematical, equal weight should be given to five other types of intelligence: musical, spatial, bodily-kinesthetic, intiapersonal, and interpersonal. Reflecting this theory, The Oregon Assessment contains items in all these areas. Gardner's theory of multiple intelligences has great heuristic implications. Assessment and curriculum developed to reflect this theory, would radically alter common educational practices. Children who typically have difficulty in school because their primary and strongest intelligences are not deemed relevant to the curriculum would have opportunities to demonstrate competence. It would make assessment and curriculum practices more "authentic" because it would be reflect more 
closely the way the world outside of school operates (Brandt, 1993). People with great interpersonal intelligence often are highly successful in political or management positions, although they might not do well on logical-mathematic tasks, or on paper and pencil tests. This same strength may actually work against a person in a typical school setting where there are few opportunities to demonstrate interpersonal skills that do not break school rules. Changing the nature of assessment and curriculum towards multiple intelligences would provide more possibilities for success for more children; a clearly positive change.

A theoretical construct such as multiple intelligences can help drive positive changes in educational practices, but change does not happen when educators continue to use tools derived from the established theories. Their current tools must be replaced by new tools for the changes to be institutionalized. The oregon Assessment has the potential to be one such tool for implementing practices reflective of multiple intelligences. Gardner recognized the importance of changing assessment practices to foster changes in curricula practices. ...Assessment should take place, insofar as possible, in the context of normal daily activities of learning; over the course of a youngster's education, assessment in contezt by others and by oneself should become a regular, increasingly automatic part of the educational experience. (Gardner, 1991, p. 204)

Following these ideas, The oregon Assessment was developed as an on-going tool to integrate assessment and teaching through naturalistic observation. 
Ironically, Gardner failed to follow his own guidelines. With Wexler-Sherman and Feldman, Gardner developed an assessment system, Project Spectrum (Wexler-Sherman, Gardner, \& Feldman, 1988). Project Spectrum utilizes a variety of preconceived materials and activities for the assessment of children rather than the child's actual educational environment. It also involves extensive use of a highly technical recording and coding system of videotaped observations. These factors ensure that assessment remains in the hands of "experts" - continuing the decontextualization of assessment from teaching and learning. In The oregon Assessment all items are based on observation in the classroom or home during typical routines and play with existing, familiar materials. Like so many assessment tools examined by the author and his colleagues for use in early childhood programs, Project spectrum initially looked promising but turned out to be highly problematic. This frustration gave impetus to the development of The oregon Assessment. Arguably, it is potentially a more functional tool for assessing multiple intelligences (and ultimately for helping teachers implement a curriculum based on multiple intelligences) than Gardner's own tool. It puts assessment directly into the hands of teachers and provides a relatively easy, articulated, and on-going process for assessment, goal-setting, and individualized curriculum planning and implementation. 
Developmentally Appropriate Practices

Few in early childhood education would disagree that NAEYC's publication, Developmentally Appropriate Practices (Bredekamp, 1987), has had more influence on the field than any other single concept or document in the past fifteen years. The field's leading professional organization set forth a series of principles for best practice that has been adopted, used, misconstrued, interpreted, argued over, and so forth, but generally widely accepted. It has set a standard and provided a basic and common language for the field. The principles greatly influenced the development and structure of The oregon Assessment. The assessment was conceived, as part of its purpose, to help implement developmentally appropriate curriculum as assessment tends to drive curriculum (Shepard, 1991).

Some of the principles of developmentally appropriate practices that are reflected on the assessment are: (a) all areas of development are provided for in the curriculum, (b) curriculum planning is developed from observations of children's interests and developmental progress, (c) the curriculum involves active exploration and interaction, (d) multi-cultural and non-sexist experiences and material are provided, (e) adults are responsive to children's individual learning styles and abilities, (f) there are many opportunities to communicate, ( $g$ ) positive self-esteem is facilitated and, $(h)$ parents are included and involved meaningfully in the program (Bredekamp, 
1987, p. 3-12). The principles also include a section specifically on assessment issues which are discussed later. The oregon Assessment includes ten different areas of development. It is an observational tool designed to help focus teachers observation on the more important aspects of development for healthy functioning (Hill, 1987; Katz, 1990, 1991), it includes a learning style inventory on the Individual Child Planning Form (Appendix B) and the assessment itself has many items that indicate learning style and developmental level of functioning. There are many items related to communication including five specifically in the communication domain (all emphasizing communication rather than speech or articulation), there are twelve items specifically under the domain of selfconcept and other items related to self-concept in other domains such as, "Willingly challenges own strength and agility", under the motor domain (item \#36). Parent input is required. The User Manual includes a section called, "the vital role of the parent(s) in collecting data and scoring" (pp. 8-9). This section details the importance of parent input and suggests ways to gather it and use it in the assessment, goal setting, and curriculum planning process. Sample completed forms with examples of parent input are included in the manual (p. 26a20 b). In these ways, key aspects of developmentally appropriate practice are incorporated into the assessment directly and indirectly, formally and informally. 
Dispositions

The importance of promoting and assessing dispositions in early childhood education is a concept developed by Katz (1990, 1991). She argues that the development of certain dispositions, such as the disposition towards being a reader and a having a positive disposition towards school and learning, are at least as important as the development of knowledge and skills. The heuristic implication is that when curriculum practices ignore the development of such positive dispositions and focus primarily on skills and knowledge (as is typical), children often develop certain negative dispositions. This can then impact the child's future development and functioning. For example, many approaches to reading development involve numerous tedious tasks and place children under pressure to achieve specific reading skills. As a result most children develop the ability to read, but many do not develop the disposition to be readers. If skills are not used, it is not much different in effect than not having them. Katz believes that effective early childhood programs must be deliberate in developing in children specific dispositions. They must be an explicit part of the curriculum. Inherent in this concept is that important longterm goals for children (i.e.; enthusiasm for learning) must not be sacrificed for a short-term gain (i.e.; the development of a specific skill).

observing, assessing, setting goals, and planning curriculum related to dispositions is an important strategy for making them explicit in practice. The oregon Assessment was 
developed, in large part, to guide teachers towards the consideration of particular dispositions as important educational goals. One of the domains included on the assessment is dispositions which includes the engagement in and enjoyment of a variety of creative activities, curiosity, willingness to try new activities, and risk-taking (items \#3744). In addition many other items incorporate dispositions towards certain activities. For example, "Actively engages in and enjoys fine motor activities (drawing, painting, puzzles, cutting, using manipulatives)" (item \#34) and "Shows interest in print medium - enjoys looking at books, asks, what signs say, etc." (item \#57). The focus of these items is on the positive disposition towards engaging in the activities rather than on skill levels.

\section{Appropriate and Effective Assessment}

The assessment of young children has come under a great deal of criticism in recent years because of the potential and real negative impact that inappropriate assessment tools and misguided assessment practices can have on children (California State Department of Education, 1989; Gifford \& O'Connor, 1992; Kamii, 1990; Meade-Roberts, 1988; Meisels, 1987; NAEYC, 1988; NASBE, 1991; NASP, 1991; SACUS, 1990). (This negative impact includes children being prematurely labeled or mislabeled, misdiagnosed, retained at grade inappropriately, misplaced in treatment programs, and/or subjected to inappropriate curriculum practices. Numerous educators and educational leadership 
organizations have called for the end to current assessment practices and have recommended guidelines for more effective and appropriate practices (Bredekamp, 1987; California State Department of Education, 1988; Gammage, 1991; Gifford \& O'Connor, 1992; Kamii, 1990; Meade-Roberts, 1988; Meisels, 1987; NAEYC, 1988; NASBE, 1991; NASP, 1991; SACUS, 1990; Walton \& Nuttall, 1992; Wexler-Sherman, Gardner, \& Feldman, 1988). Common guidelines that emerge from the various reports and research studies include: (a) the use of contextualized, observational assessment tools for authenticity; (b) the assessment of a wide spectrum of abilities, skills, and behaviors across many developmental domains; (c) the use of multiple sources of information of which formal assessment is one part; (d) input from parents; (e) limiting the use of assessment results specifically to the purposes for which they are designed; (f) the use of assessment results for individualized curriculum planning and; $(g)$ assessment in the child's native language. The oregon Assessment incorporates these recommendations. Prior to the dissertation research, it clearly met the criteria as a contextualized, observational tool that measures a broad array of abilities and behaviors across ten different domains.

\section{Context and Authenticity}

The call for contextualized assessment stems from the concern that when test items and/or children are removed from common, familiar, and typical experiences and settings, findings tend to be inaccurate and biased. This involves three aspects 
of traditional standardized, psychometric assessment: (a) items that are strange and lack meaning for the child, (b) items that are disconnected from typical curriculum practices, and, (c) direct questions or commands given by a stranger in a space other than the classroom or home. For example, common items on assessment tools for young children include "stack six one inch blocks" and "complete the sentence, 'an elephant is big and mouse is '" (McCarthey, 1972). The items are presented outside of any context, as blocks are not being stacked for a reason (even for play) and there are usually no elephants or mice to be seen anywhere, especially outside the child's own particular context. This will usually adversely affect performance. A child may often stack blocks as part her/his daily play but not be able do it (or do it as well) under the artificial conditions. A child may know the concepts of big and little, but be confused by non-existent elephants and mice or find the particular phrasing foreign and be unsure what the questioner wants. (It should be noted that although most young children will perform more poorly on decontextualized tasks than authentic, natural tasks, some may do better, perhaps because they perform well under pressure or are particularly eager to please. In either case, decontextualization tends to produce inaccurate results). This problem is best avoided when assessment is done by observing children while they are engaged in self-selected, familiar, and typical activities. Items on The oregon Assessment include: "Listens to and contributes to group discussions at mealtimes a sharing time" (item \#28) and 
"Chooses activities during free play and can stick with them for 10 minutes or more" (item \#48). To meet the criteria of the items, behaviors must be observed during typical preschool or home activities and routines:

Moving from standardized, expert (stranger) administered tests to observational instruments alone does not assure contextualization. Many observational instruments reviewed by the author include decontextualized items such as those previously discussed. These/ lead teachers to directly administer the items, as they can never be seen naturally. The teacher, rather than a stranger administering the items may be an improvement, but the problem remains that the items fail to provide meaningful information that can be used to effectively alter curriculum. They are decontextualized from curricula practices. In some cases the use of decontextualized test items eventually leads to negative changes in the curriculum to more closely match the nature of the items, approximating "teaching to the test".

observation is a vital|component of effective assessment as a strategy in obtaining authenticity of results. A great deal of research has been done on observational assessment demonstrating its efficacy as an evaluation method than can be highly valid and reliable (Airasian, 1991; Barnett, Macmann, \& Carey, 1992; Bricker, Bailey, \& Slentz, 1990; Bronson, 1994; Cunningham, 1981; Hicks, 1988; Mayes, 1987; Schaefer \& Edgerton, 1978; Turner \& Boulter, 1981). Given that, when well constructed, observational assessments are effective, their role 
in assuring greater autnenticity makes a compelling case for their use over non-observational tools. When children are observed engaging in familiar activities in familiar settings they tend to be functioning at their optimal level of performance (assuming they are not ill, hungry, etc.). The information gathered then presents a picture of the child at her/his best and can be readily used to alter practices, change materials, and employ other curricular strategies to challenge the child or remediate concerns. The link between assessment and teaching/learning is more obvious and direct than with nonobservational tests. The Oregon Assessment is designed and worded so that if it is not used observationally, it is being overtly misused. Guidance throughout The User Manual (Appendix C) provides further instruction on it use as an observational instrument.

\section{Developmental Range}

Best practices in early childhood assessment, as previously discussed, also call for the assessment of a wide spectrum of abilities, skills, and behaviors across many developmental domains. This is in response to the concern that most traditional assessments focus primarily on the cognitive domain or on a narrow band of skills across only a few domains. The constructs of human development and pedagogy that have influenced the formation of the oreaon Assessment, previously delineated, help to counteract this concern. The ten domains on the assessment include three types of cognition: problem solving, math, and literacy. Also included are social, self- 
help, self-concept, motor, dispositions, and play. The last two domains are rarely found on early childhood assessment tools and most tools include six or fewer domains.

\section{Multiple Sources and Parent Input}

The Oregon Assessment uses at least two sources of data teachers and parents. This makes it responsive to two principles of appropriate and effective assessment: multiple data sources and input from parents. The User Manual indicates that data must be collected from parents and explains a process for doing so (pp. 8-9). In addition, the individualizing forms ask for parent input on children's strengths and interests for goal setting and planning effective educational strategies. This process was described previously.

It can be used with other assessment tools, such as behavioral checklists, speech and language instruments, and health measures, for maximal benefit. In addition it can be easily accompanied by a portfolio containing concrete documentation for the items, such as photographs, drawings, anecdotal notes, audio tapes of language in use or singing, videotapes, examples of writing, etc. Portfolio items can be coded to the assessment item numbers (Grace \& Stores, 1992).

\section{Specificity of Use}

During training and in the User Manual the purposes of the assessment are clearly delineated as well as what it is not to be used for. Its main purpose is for individualized curriculum planning. Its secondary purposes are to: track progress, 
exchange information with parents, and provide additional information for referrals. It is not to be used for screening or diagnosis.

If proven to be valid, reliable, and useful, it has the potential to be used for program evaluation and other research purposes. In these cases it is almost always done, if done correctly, using a random sample of populations for comparison group or correlational studies. Individual scores are not revealed or used for other purposes. The oregon Assessment is not designed, nor should ever be used for the purpose of making placement decisions. This is clearly indicated in The User Manual (p. 7).

\section{Curriculum Planning}

Observational assessments must also be useful for educators for curriculum planning. Being observational in nature does not assure this. A case in point is the Bronson Social and Task Skills Profile (Bronson, 1994). Like Project Spectrum, it involves a sophisticated system of observation and coding techniques. Bronson's research shows it to be a valid and reliable tool, but its utility is limited to use as a research instrument. The typical classroom teacher would find the assessment cumbersome to administer and could not easily use its results for curriculum planning. It is too time consuming and involves too much technical detail apart from teaching.

The oregon Assessment is designed to assist teachers in developing individualized curriculum plans as it is administered by teachers. They then use the individualizing forms to take 
assessment information and set individual goals for children which are translated into curriculum plans. Goals can be taken directly from the assessment items because they are so closely tied to the context of children's functioning and best practices in early childhood classrooms. If a child scores low on an item such as, "Verbally expresses feelings and needs during interactions with others" (\#24), a goal can be set such as: "Child will verbally express feelings and needs during interactions with others". Curriculum strategies, formal and informal, for achieving that goal based on the strengths and interests of the child are then developed by the teacher. The utility study is designed, in part, to determine the effectiveness of The Oregon Assessment, in its current form, for curriculum planning purposes.

Use of the Child's Native Language

The User Manual indicates explicitly that all language/communication items are to be assessed in the child's native language by an observer who is fluent in that language (p. 5). Because the assessment focuses on communication in context and does not involve direct commands or asking questions of the child, the issue of native language use is a somewhat less salient than on more typical assessments. Going a step further than the recommended guidelines, native language is broadly defined to include dialects and non-verbal languages. It has been translated into spanish for use in bilingual or monolingual Spanish speaking programs and with Spanish speaking 
families. These issues are discussed more fully in the following section.

Equitable Assessment

Testing practices for young children have come under a great deal of criticism for culture, race, gender, and class bias (California State Department of Education, 1988; Cummins, 1989; Ginsburg, 1972; NAEYC, 1988; NASBE, 1991; Sosa, 1990). Bias can exist in the test items themselves, in the scoring mechanism, in the procedures used to test children, or in all three. Eor example, many standardized tests fail to consider the level of language ability a child may have in her or his native language or dialect, or functional ability in a language that combines elements of both standard English and the child's native language/dialect. Additionally many children of color and those from low-income families, often have fewer experiences that prepare them for skills needed in test-taking. Gender differences in communication styles, moral constructs, perceptions, and cognition have been well documented, even in very young children (Brooks-Gunn \& Matthews, 1979; Eder \& Hallinan, 1978; Esposito, 1979; Gilligan, 1982; Gleason \& Greif, 1983; Goodwin \& Goodwin, 1987; Lever, 1978; Maltz \& Borker, 1982; Sachs, 1987; Sheldon, 1990; Tannen, 1990; Thorne, 1986) and these differences may lead to differing interpretations of test questions and/or differing responses to test situations that will impact test scores (Bowman, 1978; Ramsey, 1987). Norms that do not reflect the population of children being 
tested often lead to scores that underestimate their true capabilities (California state Department of Education, 1988; Loyola, McBride, \& Loyola, 1991; Meisels, 1987). Most studies of testing bias examine formal, psychometric, standardized I.Q. or achievement tests that are administered directly (De Leon, 1990; Ginsburg, 1972; Loyola, MCBride, \& Loyola, 1991), not assessments that are done through naturalistic observation. For example, direct assessment can lead to a variety of problems when used with Hispanic children (Loyola, McBride, \& Loyola, 1991). The child may lack the ability to fully understand directions or the items themselves and the child may lack verbal fluency if administered in the child's second language. Even when Spanish language translations are used, certain words may have different meanings in different dialects.

\section{Relationality}

One particular attribute, relationality, seems to apply to children from many cultures outside of the dominant U.S. culture (Hale, 1983; Saracho \& Hancock, 1983; Tafoya, 1983) as well as to females (Gilligan, 1982; Tannen, 1990). Eor these children, relationality (also called field-dependence, cooperation, or affiliation, depending on the researcher and the field of study) is indicative of their approach to situations, tasks, and concepts, as opposed to the more mainstream independent and analytic approach. An Anglo adult test-giver is likely to assume that all children have a field-independent cognitive style. When this test-giver interacts with a child from a co- 
culture whose cognitive style is more relational, the child may view the test-giver as uncaring and rejecting. This increases anxiety, already high due to the presence of a stranger or strange situation, and may lead to noncompliant behaviors or inaccurate responses. If the child is from a co-culture that teaches children to obey and respect adults, as many do (Kitano, 1983; Saracho \& Hancock, 1983), the child can experience great conflict over how to please the adult test-giver while not fully understanding the task to be completed or the question to be answered.

A number of items on The oregon Assessment value the attribute of relationality. These include: "Trusts adults who are reasonably consistent and supportive" (item \#9), "Accepts comfort from others" (item \#10), "Makes eye to eye contact (providing this is the cultural norm)" (item \#11), "Shows appropriate caution with strangers" (item \#12), "Negotiates effectively with others" (item \#22), and "Displays positive relations with adults, including a balance of dependence/interdependence/ independence" (item \#26). The emphasis on the social domain throughout the assessment reflects respect for children from relational co-cultures and this can lead to more positive outcomes for them. In an ethnographic study, Carrasco (1979) documented that when an Anglo teacher focused on the social competence of an Hispanic kindergarten child (who the teacher initially viewed as low achieving) she began to see the child more favorably (improving their relationship), altered her instructional strategies, and 
increased her expectations, leading to improved student performance. The item -- "Shows acceptance of people who are different from her/himself (disabilities, gender, skin color, etc)" (\#27) -- helps teachers focus on developing a classroom where differences are appreciated.

Towards an Equitable Assessment Tool

Anxiety, lack of understanding, assumptions, conflicting messages, misinterpretation of cultural-based behaviors, and other factors discussed above will lead to lower test scores in almost all cases and therefore to biased results. When the purpose of a test is to determine placement in a program, promotion to the next grade level, or a similar "high stakes" outcome (Meisels, 1987), it is imperative that tests not reflect culture, race, gender, or class biases as misplacement can lead to severely damaging consequences that may last a life-time (Meisels, 1987; Shepard, 1991; Shepard \& Smith, 1986). Because of the difficulty of removing bias from tests and the over representation of minority groups in special education programs (Oakes, 1985) many educators are calling for the end of standardized testing for children below fourth grade (Kamii, 1990; NAEYC, 1988; SOSa, 1990).

The Oregon Assessment should avoid many of the aforementioned problems that can negatively impact equitable testing of children. Because it is an assessment of children as they are observed naturalistically, problems caused by direct, formal assessment procedures are not an issue. The teacher administers the assessment and does not askldirect questions or 
seek narrowly defined "correct" responses. Stranger anxiety and miscommunication due to intercultural factors are minimized. Many educators are calling for more use of this type of assessment and eliminating or reducing formal assessments as observational assessments often give more accurate, useful information (Elores \& Riley, 1982; Kamii, 1990; NASBE, 1991; SACUS, 1990).

The purpose of The oregon Assessment is for curriculum planning. Its use for "high stakes", as previously discussed, would be a misuse of the instrument. When used for research purposes, data collection would entail confidential sampling, assuring that it will not be used to judge individual children for placement purposes. Because it is a criterion-referenced instrument, comparisons to an unrepresentative group (normreferencing) is not possible.

Language ability, language differences, and bilingualism have little negative impact on scores for children from cocultures on The oregon Assessment, because most of the language items deal with communicative competence rather than fluency or understanding. Competence can be expressed in any language or dialect (including non-verbal languages such as American Sign Language) as observations are focused on the production of desired outcomes through language. Only when the child being assessed is unsuccessful at communicating with a peer or adult who speaks a different language or dialect than the child or who cannot understand the child's attempts at speaking the language of the peer or adult is there a possibility that scores would be 
affected. The item, "Communicates so $\mathrm{s} /$ he is understood by others" (item \#31), is the most problematic in this regard. To deal with this, guidelines in the user manual for language items specify that the observations should be conducted when the child is conversing with a receiver who is fluent in the same language or dialect the child is using (p. 5).

Numerous experts in the area of intercultural education stress the important role teachers have in helping children achieve biculturalism, the ability to live successfully in their own culture and the mainstream culture reflected in schools (Albert \& Triandis, 1991; De Leon, 1990; Saracho \& Hancock, 1983). Rigg and Allen (1989) recommend using accepted constructs of development when working with children from nondominant co-cultures. Their premise is that all children move through the same developmental stages and they are children first and foremost with similar needs as other children. Gonzalez-Mena (1992) states that "it is never all right to go along with sexism, oppression, or abuse, even if you are told that it is cultural" (p. 8). For these reasons items that may conflict with certain values of some co-cultures should not be eliminated but teachers should be made aware of the possible cultural basis for the observed behaviors and show sensitivity when sharing this evaluation with parents. For example, the item, "Displays flexibility in roles - can be a leader and a follower and does not adhere rigidly to a sex role" (item \#23), may clash with values in some families from certain Hispanic cultures. A lower, score for a child from such a family may be 
an accurate assessment, but the comment area should include information about cultural/familial expectations for that child. Goals and strategies developed from the assessment for helping a child move beyond rigid roles also need to consider the cultural/familial expectations. Specific guidelines to this effect for this item are included in The User Manual (p. 16). The introduction to the manual contains general guidelines for administering the assessment in a culturally sensitive manner. The Spanish version of the assessment when used by Spanish speaking teachers and/or parents helps create mutual respect and affiliation, factors that are critical to positive and effective parent/community/school relations. The User Manual informs the user that the Spanish translation is in a Mexican-American dialect and may need revision for other Spanish speaking populations. It also includes a strong recommendation to teachers not to assess Hispanic children and children from other co-cultures until they feel comfortable in the classroom and with the teacher.

The oregon Assessment holds great promise as a useful and effective tool to equitably assess all children. Because it is designed to be used for the specific purpose of curriculum planning through naturalistic observation, it should be less likely to produce biased results or harmful outcomes to children than traditional, formal psychometric assessments. The utility research will attempt to determine this. The Spanish language version should assist in creating positive affiliation with Hispanic families who highly value their language. 
Head Start Assessment

As discussed in Chapter I, The Oregon Assessment was developed for and with the Head start community. It is linked closely to the needs, values, and culture of Head start. There are two main areas of inquiry related to assessment in Head start that are explored in this section. The first is the issue of measuring and promoting social competence in Head start children, and the second is the issue of evaluating the efficacy of the Head start program itself.

Social Competence as an Educational Goal for Children from Low-Income Eamilies

Although the importance of helping children develop social competence was discussed previously, this is particularly important for children from low income families, the population served by Head start. Young children from families of low socio-economic status may be at greater risk for failure due to poor social stills, as numerous studies have revealed. When these children are compared with other children, they engage in less dramatic play (Eein \& Stork, 1981; Griffing, 1980; Rosen, 1974; Smilansky, 1968; White, 1978), more parallel and solitary play (Murphy \& Wilkinson, 1980; Rubin, Maioni, \& Hornung, 1976), and more passive (non-interactive) time with adults (Murphy \& Wilkinson, 1980). Fortunately, there is some evidence that when given an opportunity to interact with peers in good quality preschool settings integrated by socioeconomic status (a rare occurrence in Head Start), children from low socioeconomic 
families showed no differences in play behaviors from other children. (von Zuben, Crist, \& Mayberry, 1991).

One example of the great positive impact of attending to the social domain for children from low-income families is provided by the work of Comer (1981). He developed a public school model that is highly successful in dramatically improving the academic performance and morale of children in schools with traditionally poor academic performances, high crime rates, and high drop-out rates, all characteristics associated with schools serving low income families. His focus for reform was not on academic rigor, but on the social and emotional climate. A team of teachers, the principal, and parents were empowered to make decisions at the individual school level. An active social support system is at the heart of the plan. Comer stated,

In an individualistic society such as ours we like to believe that we accomplish everything on our own. But we forget that the talented flourish because others create the climate for them to do so, and that we are motivated by an invisible web of kin, neighbors, teachers. Children internalize this support in their attitudes, values, and ways, and so do parents. (cj.ted in Louv, 1990, p. 350-351)

Assessment that focuses on and fosters social and emotional competence has the potential to be an effective and useful tool for the children served by Head start.

The Measurement of Social Competence for Head start Erogram Evaluation

As described previously, the oregon Assessment is an instrument developed in part by Head start staff directly from a need to measure aspects of development and child functioning reflected in classroom practices and the goals that teachers 
have for children, primarily social and emotional. This need is further documented by Raven and Zigler (1991), who describe the historic and current failure of the Head start administration to measure the stated central goal of Head start,! social competence. The authors call for the development of an instrument that can provide such measures. Numerous researchers and program developers have concurred that the goal of Head Start for children should continue to be the development of social competence and that Head start research has historically failed to adequately measure this goal (Cole washington, 1986; Gordon, 1979; McKey, 1985; Raizen \& Bobrow, 1974; Schweinhart \& Weikart, 1986). This development of this assessment is, in part, in response to the concerns raised fromi within and outside of the Head start community.

The enthusiastic response from numerous/Head start programs for the instrument begins to reflect its potential to measure what Head Start staff believe is most important (Sweeney, 1995). The determination of its validity, reliability, and utility will allow for its use as a research tool in helping to demonstrate the efficacy of Head start. If Head start is to fully realize its goal of developing social competence in young children and providing evidence of its effectiveness at doing sa, a reliable and valid assessment tool that can adequately measure the social/emotional domain will be required. The oregon Assessment has the potential to be one such tool. 
Conclusion

The Oregon Assessment was developed from a need for staff

from Head start and other early childhood programs for an assessment tool that would be useful to classroom teachers in planning individualized curriculum. The tool needed to be more responsive to a variety of concerns and constructs than the tools they were using. They sought an observational, contextualized, authentic assessment that measured social and emotional functioning as well as cognitive processing and certain skills and abilities. Additionally they wanted it to meet the criteria for effective, appropriate, and equitable assessment and be reflective of best practices in early childhood education. If reflective of best practices, then the tendency for assessment to drive curriculum could result in a positive impact on classroom practices.

The author also hoped to develop a tool that could be used effectively for research purposes, particuiarly to assess the efficacy of the Head start program along the important dimension of social competence, the primary goal of Head start for the children it serves. In the theories of interactionalism and multiple intelligences, as well as related concepts regarding the importance of the social domain and the development of dispositions, the author found a basis for developing an approach, structure, and method for the assessment of young children. The combined influences and needs of program staff, researchers, theorists, and critics, resulted in the development of The oregon Assessment. 
The literature review discussed the theory of interactionalism as an important construct for promoting effective education and development of young children. It gave the assessment its characteristic emphasis on social development and functioning and impetus for the inclusion of play as a domain. The concept of the zone of proximal development provided a basis for scoring the assessment based on how much assistance is provided by the teacher (as well as frequency). The importance of focusing on the social domain in education was discussed, providing further justification for this emphasis in the assessment. Gardner's theory of multiple intelligences influenced the inclusion of ten different domains on the assessment and the inclusion of items related to all seven intelligences: logical-mathematical, linguistic, musical, spatial, bodily-kinesthetic, interpersonal, and intrapersonal. The items selected and the approach used by The oregon Assessment were also influenced by key principles of developmentally appropriate practice, including the importance of observing and responding to children's individual needs and learning styles, learning through active exploration and interaction, promoting múlti-cultural and non-sexist experiences, and fostering communication. Promoting certain positive dispositions as an educational strategy and goal, provided the impetus for the inclusion of observing for dispositions towards creativity, curiosity, risk-taking, and other dispositions. The principles of effective, appropriate, and equitable assessment practices for young children drove much 
of the design and structure of the assessment as an

observational, contextual, authentic, criterion-referenced tool that requires parent input, to be used primarily for individualized curriculum planning. The need for an effective tool for Head start evaluation was also discussed. As an assessment that focuses on social competence, The oregon Assessment has the potential to fill this need.

The dissertation research was undertaken to establish various types of validity, reliability, and utility for The oregon Assessment across a variety of dimensions. If proven to be valid, reliable and useful, the assessment would then have credibility as a research tool and an instrument for use in classrooms for individualized curriculum planning. In addition, information from the research will be used to refine the assessment to make it more effective for its intended purposes. 
CHAPTER III

METHODOLOGY

Introduction

An effective measure of children's functioning must include several key components. It must be valid in that it clearly measures what it purports to measure and it must be reliable in that its measurements are consistent, stable, and accurate (Berk, 1980; Ebel \& Frisbie, 1991; Gullo, 1994; Wiersma \& Jurs, 1990; Wortham, 1990). It must have utility in that it is perceived by users as accurate, helpful, and cost effective to administer and score in terms of teacher time (Cangelosi, 1990; Wiersma \& Jurs, 1990). A balance must be obtained between creating a test with many items, which can increase reliability, and a shorter test which increases utility. Utility also means that it provides the user with needed and important information, the items are understandable and easy to score, and it assists the user in working effectively with children (Bricker, Bailey, \& Slentz, 1990).

Comprehensive test development research typically seeks to determine a number of different kinds of validity. A common type found in test development literature, predictive validity (Ebel \& Frisbie, 1991; Hambleton, 1980, 1984; Wiersma \& Jurs, 1990), was not used in this research. Predictive validity is sought for assessments that are designed to predict outcomes for 
children. For example, to predict at four years of age, if a child will later have trouble learning to read in first grade. Ostensibly this information is used to target intervention services to the children most in need of those services. However, The Oregon Assessment has different purposes, as previously discussed. It was developed to assess current functioning and to assist with individualized curriculum planning, rather than to predict performance. Also, if proven effective and used appropriately it should result in effective practices that would remediate areas of weakness thereby nullifying its predictive capability. For these reasons a study of predictive validity was not included in the research design. Curricula, item, construct, and congruent validity -- measures relevant to an assessment designed for individualized curriculum planning -- were included. They were obtained by surveying 15 early childhood education experts who rated each item, each domain, and the test as whole.

Three important measures of reliability were included in the research design. Perhaps the single most important measurement in demonstrating the efficacy of a test is its internal reliability or consistency determined by obtaining Chronbach's alpha (also called coefficient alpha), the splithalf correlation, the spearman-Brown coefficient, and item reliability for each item, domain, and the total score (Ebel \& Frisbie, 1991; Wiersma \& Jurs, 1990; Wortham, 1990). These measures were obtained through statistical analysis by using scores from the entire sample of 199 children. Test-retest 
reliability was measured to determine if scores remain

consistent when given by the same tester to the same child after

a short interlude to minimize the influence of maturation. This

in essence is one measure of its stability. Inter-observer

reliability was determined to ascertain the test's stability

when different testers assess the same child at approximately

the same time.

Through a survey, the utility or usefulness of The oregon

Assessment to teachers and administrators was determined.

Questions on the survey asked users to rate the assessment on a variety of dimensions, including how effective it is for use with parents, how well it helps in setting goals for children, how much time it takes to administer, and how responsive it is to diversity. Open-ended comments were solicited at the end of the questionnaire.

Both surveys -- the one used for curricular, item, and construct validity completed by 15 experts and the one used for utility completed by 114 users -- employed a 1-4 Likert-scale for ratings (see Appendices $\mathrm{E}$ and $\mathrm{F}$ ). Ratings of 1 and 2 were negative ratings while 3 and 4 were positive ratings. The lower the rating the more negative the response, the higher the rating the more positive the response. A question on the survey asking respondents to evaluate an assessment item, domain, or other issue about the assessment was considered to receive an acceptable score if the mean score was 3.0 or more and the median score was 3 or more (Berk, 1978; Hambleton, 1980, 1984). 
Sample

Curricular, item, and construct validity were determined from a questionnaire (Appendix E). Thirty questionnaires were sent to specific, targeted early childhood experts from across the United States. They were selected to represent a cross section of the field -- academicians, researchers, practitioners, consultants, etc. -- and people who would have particular expertise in both early childhood curriculum and assessment. Fifteen questionnaires were returned. This relatively low $50 \%$ return rate was expected because the survey was very lengthy and it was sent to people who are extremely busy and are usually highly compensated for their time. The experts who returned surveys included two practicing teachers (with great expertise as acknowledged by their peers as they are highly sought as trainers), three program executive directors (also highly regarded by peers and sought as trainers), four university faculty members in early childhood departments (two of whom also train and consult internationally and are regarded as national leaders in the field, all of whom publish extensively), and one consultant/writer who is also regarded as a leading thinker, trainer and expert in the field. The remaining four respondents chose to be anonymous, although only highly regarded experts were sent surveys.

Reliability and congruent validity were determined by using a representative, random sample of 200 children in a variety of preschool settings in two northwest states (199 results were usable). The settings, selected to represent 
geographic and democraphic diversity (but include a large Head start sample), included two Head start programs, a school district and a private non-profit child care center. The sample sites included Southern Oregon Head Start -- serving a multiethnic population (although primarily white) of 3-5 year old children from low-income families in urban and rural areas; Washington County Head start -- serving a multi-ethnic population (including many Hispanic children) of 3-5 year old children from low-income families in mostly rural areas in northwest Oregon; Mt. Park Play School -- serving a primarily white, high-income, suburban population in Oregon; and the Spokane, Washington School District in eastern Washington -serving a multi-racial, multi-income, urban population. This site population included a number of children slightly older than five years of age as the program uses the assessment in their kindergarten classes as well as their preschool classes. Children enrolled in Head start are over-represented in the sample because of the close link between the assessment and Head start goals and needs. If the case can be made that the assessment is valid, reliable, and useful for children in Head Start, it could have widespread use as a Head start evaluation tool (program evaluation and child functioning evaluation) and a curriculum planning tool.

Twenty children from each of the four sites were randomly selected to participate in the test-retest reliability, interobserver reliability and congruent validity studies, for a total of 60 children from each site, and 80 children for each study. 
In many cases, especially in the smaller sites, the same child participated in more than one study. Parent permission was obtained and written assurances of anonymity were provided to each parent. The children were from 54 different classrooms (all classrooms at each of the four sites were involved). The racial composition of the sample was: White- $82.12 \%$, Hispanic-7.73\%, Native American-3.86\%, African American-2.89\%, Asian-1.93\%, and Other-1.45\%. This sample is more reflective of the Northwest than the U.S. as a whole. Only programs which selected the assessment themselves as their tool of choice were included in the study resulting in this particular population distribution. This deliberate constraint resulted in less ethnic diversity among the sample, resulting in less generalizability but greater validity for the results, because the efficacy of the assessment was determined in context. It was not imposed on the users nor was an artificial researcheroriented milieu created which can skew the results.

The mean age of the sample children was 5 years as was the median age, although 4.5 years was the mode. They ranged in age from 3.25 to 6.5 years. Eorty-seven percent of the sample were males and $53 \%$ females.

For the utility study, 200 surveys were mailed. Twenty programs in eight states using the assessment were sent five to fifteen surveys each, depending on the size of the program. These programs constituted all known U.S. users of the assessment at the time. They were sent to education coordinators or directors to randomly distribute to teachers. 
The coordinator or director was asked to complete one as well. One hundred and fourteen surveys were returned (57\% return rate). The typical respondent was a Head Teacher (76.3\%) with a B.A./B.S. degree (47\%) and 7-10 years of preschool teaching experience. The respondents have been using the assessment for an average of 2.1 years and received, typically, three hours or less of training on using it.

\section{Procedures}

The process used to determine the various types of validity, reliability, and utility is described in this section. The author trained each teacher, administrator, and other staff member involved in this research study. Training typically lasted three hours and included information on random sampling, objective data collection, parental permission, coding to keep individual identity confidential, and the purpose and design of the research. It was provided on-site in southern oregon; Washington County, Oregon; Lake Oswego, Oregon; and Spokane, Washington. Three research assistants who helped administer the McCarthy scales were also trained by the author.

\section{Validity}

Four types of validity were determined:

1. Curricular validity, or the match between the assessment and curricula objectives (Gullo, 1994; Hambleton, 1980, 1984; Salvia \& Hughes, 1990; Wiersma \& Jurs, 1990; Wortham, 1990), was established from the ratings of fifteen experts as to the degree to which each test item, each domain, 
and the domains taken together as a whole reflect developmentally appropriate curricular practices for 3-5 year old children. They rated items, domains, and the assessment on four point Likert-scale: $1=$ not at all, $2=a$ little, $3=$ generally, and $4=$ strongly.

2. Item validity was determined by the fifteen experts who rated each item according to the degree that it is an important indicator of the domain of development it is under. This can also be termed item-objective congruence (Berk, 1978; Hambleton, 1980, 1984) or item analysis (Salvia \& Hughes, 1990; Wortham, 1990): The same Likert-scale was used as described above.

3. Construct validity, or the degree to which the assessment adheres to principles of appropriate and effective assessment was determined by the experts' ratings of the test in relation to those principles (Hambleton, 1980, 1984). A copy of those principles was included with the questionnaire (Appendix E). Construct validity was also determined by the manner in which items and the assessment were developed. As described previously the items and domains were developed by Head start education coordinators based on the need for a test that reflects established goals for children, best classroom practices, and would be most practical and useful for the purpose of curriculum planning, conferencing, and referral. Item and domain development are two important aspects of construct validity (Hambleton, 1980, 1984; Wiersma \& Jurs, 1990) 
4. Congruent validity, also called concurrent validity (Ebel \& Erisbie, 1991; Wiersma \& Jurs, 1990; Wortham, 1990), was determined by administering The McCarthy scales for Children (McCarthy, 1972), an individually administered developmental assessment, and the Vineland Adaptive Behavior Scales, Classroom Edition (Sparrow, Balla, \& Cicchetti, 1984), a 244 item questionnaire grouped by the domains of language, daily living, socialization, and motor skills) to 80 children within one month of the administration of The oregon Assessment. Professional educators, trained on and experienced with The Mccarthy scales administered them, and the classroom teachers administered the Vineland. In the edition of the Vineland that was used, the classroom edition, teachers are the designated administrators of the assessment. Data was analyzed to determine Pearson productmoment correlations between scores on these measures and scores on The Oregon Assessment.

These measures were selected because they are standardized, well validated, and respected measures used extensively in research (Mitchell, 1985). The McCarthy provides a measure of overall functioning but is limited as it does not include items in the social, emotional, self-help, play, or dispositions domains. The vineland has an emphasis on social and self-help skills, but is limited because it does not include items on any cognitive domain. Neither assessment was developed in response to the theoretical constructs that inform the oregon Assessment, nor do they have the unique scoring or data collection qualities previously discribed. 
Reliability

Reliability was determined using three methods:

1. Test-retest reliability is a common method of determining reliability to assess a test's stability (Ebel \& Erisbie, 1991; Salvia \& Hughes, 1990; Wiersma \& Jurs, 1990; Wortham, 1990). A random sample of 80 children were given the test a second time by the same tester within three weeks after the completion of the first administration to determine testretest reliability. Results were analyzed using a Pearson product-moment correlation.

2. Inter-observer reliability, also a widely used method to determine a test's stability, was used to determine stability across test-givers (Ebel \& Erisbie, 1991; Salvia \& Hughes, 1990; Wiersma \& Jurs, 1990). A random sample of 80 children were given the test by two different testers. One tester was the child's classroom teacher, the second tester was either a coteacher, assistant teacher, an education coordinator or program director who had been trained on using the assessment and with whom the child was familiar. The child was observed in the same environment over roughly the same period of time and testers were instructed not to share scores or information. Results were analyzed using a Pearson product-moment correlation.

3. Internal reliability is an important measure of "the degree of relationship among items on a test, indicating whether items are positively interrelated and measure the same trait or characteristic." (Wortham, 1990, p. 178). This was obtained statistically by utilizing all useable scores on The oregon 
Assessment from the entire sample $(\underline{N}=199)$. Values for Chronbach's alpha or coefficient alpha, the split-half correlation, the Spearman-Brown coefficient, and item reliability for each item, domain, and the total score were obtained (Ebel \& Frisbie, 1991; Wiersma \& Jurs, 1990; Wortham, 1990). Chronbach's alpha "provides the average correlation among all possible halves" (Salvia, 1990, p. 68) and therefore is a very powerful indicator of internal consistency. The split-half correlation is a method "for estimating test reliability by which a test is divided into two comparable halves and the scores on the halves are then correlated." (Wiersma \& Jurs, 1990, p. 404). In this case, one half of the items consists of the odd numbered items and the other half consists of the even numbered items. It is another measure of internal consistency. The Spearman-Brown coefficient measures the increase in reliability of the whole test compared to each half of the test (Wiersma \& Jurs, 1990, p. 165). All three measures determine internal consistency through a split-test procedure. Item reliability was determined by obtaining a Pearson product-moment coefficient for the items and the domains as they correlate to total scores. This is a measure of internal reliability for individual items or domains rather than the test as whole.

\section{Utility}

The utility or usefulness (Bricker, Bailey, \& Slentz, 1990) of The Oregon Assessment was determined by obtaining data from questionnaires from teachers and administrators. Two 
hundred surveys were mailed and 114 were returned. This return rate of $57 \%$ was lower than hoped, but not unreasonable. It included respondents from a wide variety of programs throughout the U.S. and provided an adequate sample size (over 100 surveys). It appears to be a fairly representational sample and the results should generalizable to all users.

The questionnaire contained 19 items, 4 of which were demographic questions (see Appendix E). Questions and items asked opinions about the accuracy of the assessment, the scoring system, the clarity of the user manual, its efficacy in helping teachers plan individualized curriculum, its usefulness with parents, its ability to respond to diversity, and the time it takes to administer. Space/ was provided at the end for respondents to make open-ended comments.

\section{Conclusion}

Through a multi-faceted research design, various measures of validity and reliability and a measure of utility across a number of dimensions were sought for The oregon Assessment. These included curricula, item, construct and congruent validity; test-retest, inter-observer, and internal reliability; and the usefulness of the instrument in terms of accuracy, assistance with curriculum planning, responsiveness to diversity, helpfulness in working with parents, and time it takes to administer. Comman and well-accepted test development procedures for sample selection and research methodology were used by the author to achieve this goal. 
The results of the research will provide information for refinement of the assessment. If it is found to have an acceptable degree of validity, reliability, and usefulness, it would be an effective tool for assisting teachers and programs to assess children and to implement an individualized curriculum. In addition researchers could avail themselves of the instrument for program evaluation purposes. 
CHAPTER IV

RESULTS

This chapter reports the results of the research related to each research question. This includes the results of the studies to determine curricular, item, construct, and congruent validity; test-retest, inter-observer and internal reliability; and utility.

The general research question was: Is The oregon Assessment for 3-5 Year olds in Developmentally Appropriate classrooms a valid, reliable, and useful assessment tool? The specific research questions were:

Research Question 1: Is The Oregon Assessment a valid tool to assess the functioning of young children three to six years of age in preschool classrooms?

Research Question 1a: Does The Oregon Assessment have curricular validity -- does it match the objectives of developmentally appropriate curriculum practices? Research Question 1b: Does The oregon Assessment have item validity -- is each item an important indicator of the domain it is under?

Research Question 1c: Does The Oregon Assessment have construct validity -- does the test reflect the guidelines for appropriate and effective assessment of young children? 
Research Question 1d: Does The Oregon Assessment have congruent validity -- do children score similarly on The oregon Assessment as on other, more established, well-researched instruments that: purport to measure similar areas of functioning?

Research Question 2: Is The Oregon Assessment|a reliable tool to assess the functioning of young children three to six years of age in preschool classraoms?

Research Question 2a: Does The Oregon Assessment have test-retest reliability? Research question 2b: Does The Oregon Assessment have inter-observer reliability?

Research Question 2c: Does The Oregon Assessment have internal reliability?

Research Question 3: Is The Oregon Assessment a useful tool for preschool teachers to effectively assess and plan for children?

Research Question 3a: Do teachers and administrators perceive The Oregon Assessment to be accurate? Research Question 3b: Do teachers and administrators perceive that The Oregon Assessment provides them with important information about children? Research Question 3c: Do teachers and administrators perceive The Oregon Assessment User Manual to be clear and understandable? Research Question 3d: Do teachers and administrators perceive that the Oregon Assessment is effective in helping to write goals? 
Research Question 3e: Do teachers and administrators perceive that The Oregon Assessment is effective for curriculum planning and tracking children's progress? Research Question 3f: Do teachers and administrators perceive that The oregon Assessment is more useful than other assessment tools that they have used? Research Question 3g: Do teachers and administrators perceive that parents respond favorably to The oregon Assessment?

Research question 3h: Do teachers and administrators perceive that The oregon Assessment is useful for helping parents understand their children and set appropriate goals for them? Research Question 3i: Do teachers and administrators perceive that The oregon Assessment is responsive to diversity?

Research Question 3j: Do teachers and administrators perceive that the completed assessment provides a rich and in-depth "picture" of a child? Research Question 3k: How long does it take to complete the assessment on each child? Research Question 31: over how long a period of time do teachers administer an assessment on a child? Research Question 3m: Do teachers and administrators perceive that The oregon Assessment takes a reasonable amount of time to administer? 
Research Question 3n: Do teachers and administrators

find the scoring system to be understandable?

\section{Validity}

The following four sections address research question 1 , Is The Oregon Assessment a valid tool to assess the functioning of young children three to six years of age in preschool classrooms? Validity was addressed through four studies to determine the curricular, item, construct, and congruent validity of the assessment. Through a lengthy survey, 15 early childhood education experts rated items, domains, and the domains taken together as a whole to determine the answer to research questions $1 a$ through $1 c$. The survey used to determine these three measures of validity employed a 1-4 Likert-scale for ratings (see Appendi: E). Ratings of 1 and 2 were negative ratings while 3 and 4 were positive ratings. The lower the rating the more negative the response, the higher the rating the more positive the response. An item was considered to have an acceptable score if the mean score was 3.0 or more and the median score was 3 or more (Berk, 1979; Hambleton, 1980, 1934). Congruent validity was determined by an extensive study that included 200 children (199 usabie scores) in four programs in two northwest states.

Curricular Validity

Research question la asked, does The oregon Assessment have curricular validity -- does it match the objectives of 
developmentaliy appropriate curriculum practices? The results of the questionnaire completed by the experts indicated that all items, each domain, and all domains taken together as a whole generally or strongly reflect the theoretical construct of developmentally appropriate practice, the essential curriculum framework for the field and well-known by all respondents. None of the 00 items received a mean score of less than 3.0 or a median score of less than 3. (A score of 3 indicated general agreement that the item or domain reflects the theoretical construct of developmentally appropriate practice and a score of 4 indicated strong agreement). Of the 60 items only five received a mean score of less than 3.5 and only four received a median score of 3 rather than 4 . The four items rated lower than the others, having a median of 3 and ranging from a mean of 3.0 to 3.2 , were item \#4, "Puts material in proper place when finished"; item \#8, "Cares for personal safety - uses seat belt, is not reckless on playground, in classroom, and on field trips"; item \#11, "Makes eye contact (providing this is a cultural norm)"; and item \#12, "shows appropriate caution with strangers".

Eor the ten domains, the mean scores ranged from 3.47 to 3.87 and the median scores were all 4. All domains taken together received a mean score of 3.70 and a median score of 4 , indicating quite strong agreement that each domain and the domains together reflect developmentally appropriate curriculum practices. See Table 1 below. 
Table 1

Experts' Ratings (Based on a 1-4 Likert-scale) of Curricular

Validity for the Ten Domains and All Domains Taken Together $(\underline{\mathrm{N}}=15)$

\begin{tabular}{llll}
\hline & Mean & Median & SD \\
& & & \\
Domain & & 4 & .46 \\
Play & 3.73 & 4 & .64 \\
Self-help & 3.47 & 4 & .64 \\
Self-concept & 3.47 & 4 & .35 \\
Social & 3.87 & 4 & .46 \\
Communication & 3.70 & 4 & .49 \\
Dispositions & 3.67 & 4 & .59 \\
Motor & 3.73 & 4 & .59 \\
Cognitive-problem & 3.73 & 4 & .62 \\
$\quad$ Solving & 3.67 & 4 & .63 \\
Cognitive-Math & 3.60 & 4 & .43 \\
Cognitive-Literacy & 3.67 & & \\
All Domains Together & 3.67 & & \\
\end{tabular}

The 15 experts were encouraged to write open-ended comments on the back of the survey. Many also wrote comments next to items, domains, and other places on the survey. The following is a summary of the comments related to curricular validity.

- One indicated that domain of dispositions was particuiar important for curricula validity and appreciated its inclusion on the assessment.

- There were eight generally positive comments. One stated, "This is an outstanding piece of work. I hate most assessment forms but finally yours has come upon the scene. It is REALLY GOOD." Another said that she or he is very interested in using it. One respondent expressed 
empathy with the task, "developmentally appropriate assessment is a challenge if not an impossibility". Negative comments tended to be specific and are delineated below.

- One respondent gave extensive and specific feedback about typos and lack of clarity in The User Manual.

- One respondent indicated that there should be a separate domain for emotional development as differentiated from self-concept development.

- One stated that it is long and wanted to know what teachers have say about that.

- One respondent was concerned that children who are "watchers" or who think their thoughts rather than verbalize them would score lower. She felt that this is a learning style difference and should not be reflected by lower scores.

- One suggested strengthening the math, literacy, and motor sections and recommended the inclusion of specific items such as a measurement of spatial relations, application of math concepts, knowledge that print carries meaning, attempts to write own messages, and the ability to make objects with purposeful control such as Lego constructions.

- One suggested that if it included a few more difficult items in math and literacy, it would be very appropriate to use with kindergarten age children which includes si: year olds (indicating some possible curricular validity 


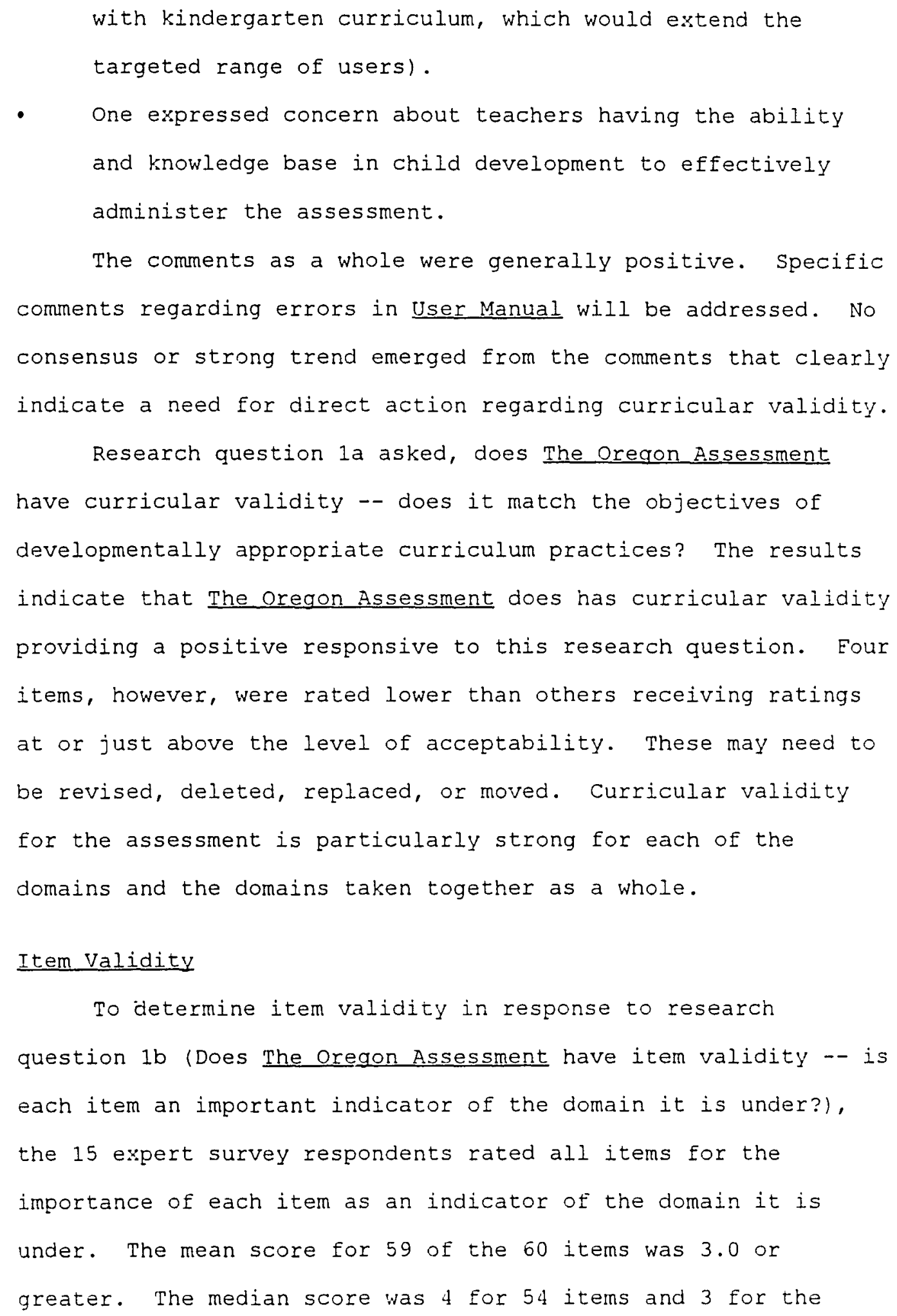


remaining six items. One item, received a mean score of 2.47 and a median score of 3 indicating low item validity. This was item \#4, "Puts mațerials in proper place when finished." Respondents likely viewed this as more related to the self-help domain then the play domain. The play domain would be strengthened by replacing this item with an item more directly related to play skills or behaviors. The other items receiving median scores of 3 (although receiving acceptable mean scores of 3.0 or greater) included item \#3, "Is a self-directed, motivated learner -- shows initiative in finding and choosing activities"; item \#5, "Uses language skills to assist in directing cooperative (dramatic) play"; item \#8, "Cares for personal safety -- uses seat belt, is not reckless on playground, in classroom, and on field trips"; item \#11, "Makes eye to eye contact (providing this is a cultural norm)"; and item \#12, "Show appropriate caution with strangers". Because items 4, 8, 11, and 12 received lower curricular validity ratings as well, they need to be modified, moved to another domain, or eliminated on future versions of the test. This issue will be addressed further in Chapter $V$. There were no comments on the survey related to item validity.

Except for one item, research question 1 b regarding the item validity of the assessment was affirmed. There was an acceptable degree of item validity for all items but \#4, "Puts materials in proper place when finished." Several other items were rated lower than others with ratings just at or slightly above the level of acceptability. of these items, four items 
(including item \#4) were also the lowest rated items for curricular validity. Eor this reason these four items (4, 3, 11, and 12) should be reconsidered when revisions to the assessment are made.

\section{Construct Validity}

Research question 1c asked, Does The oredon Assessment have construct validity -- does the test reflect the guidelines for appropriate and effective assessment of young children? Construct validity was addressed in two ways, through the process of how the assessment was developed and through one item on the survey completed by the 15 early childhood experts. As previously described, the assessment was developed from requests from practitioners for an assessment tool that reflects best curricula and assessment practices as identified by leading professional organizations in the field and that could be used effectively for individualized curriculum planning. It was developed in collaboration with fifteen education coordinators and was field tested in programs and revised five times. This context-bound development process, different than the usual process of test development that occurs in isolation from practitioners, often based on previously developed assessments, provides a partial but compeliing rationale for construct validity (Berk, 1978).

In addition, the fifteen experts rated how adequately the assessment as a whole reflects principles of appropriate assessment as outlined in the 1991 NAEYC position statement, Guidelines for aporopriate curriculum content and assessment in 
program serving children ages 3 through 8 . A copy of the guidelines was attached to the survey (see Appendiz E). It received a mean score of 3.60 on a 1-4 Likert-scale, with 3 indicating that it generally reflects the guidelines and 4 indicating that it strongly reflects the guidelines. The median score was 4, indicating quite strong agreement that the assessment reflects the guidelines. This, along with the organic process of its development, provides a strong case for construct validity.

There were only three comments from the 15 respondents related to construct validity. Two expressed concerns related to culture and diversity issues. One of these stated that some items value behaviors that may not reflect cultures of family styles. Another said that because of its focus on dispositions and social/emotional functioning it is too value-laden and therefore culture-bound. However this same respondent indicated that he or she supports the values represented by the assessment and has not resolved the dilemma of assessing across cultures. The only other comment related to construct validity was from one respondent who felt that cognitive math items are problematic because they would likely lead teachers to do direct testing rather than observation. The valid concerns raised by these comments are addressed in Chapter $V$.

Research question 1c, regarding the construct validity of the assessment, was answered affirmatively. The assessment, through its development process and from the positive response from early childhood education experts to the survey item, has 
an acceptable level of curricular validity. The comments raised concerns, however, about the issue of its responsiveness to diversity and the possibility that math items may lead to direct testing.

\section{Congruent Validity}

Research question 1d asked, Does The oregon Assessment have congruent validity -- do children score similarly on the oregon Assessment as on other, more established, well-researched instruments that purport to measure similar areas of functioning? An extensive study using a sample 200 children (199 usable scores) in four programs in two northwest states found that Pearson product-moment correlations between total scores on The Oregon Assessment and The Vineland Scales were $.460(p<.01)$. Between total scores on The oregon Assessment and The McCarthy Scales the correlation was $.395(\mathrm{p}<.01)$. This indicates a moderate, but significant correlation between The Oregon Assessment and these scales. Comparisons between any of the subtests of The Oregon Assessment and any subtests of the other assessments revealed weaker correlations than comparisons between the total scores. Table 2 delineates the correlations between selected subtests of similar domains and between total scores. Moderate correlations were expected as the procedures and purposes of the assessments are different, especially for The McCarthy Scales, which is a formal, non-observational, normreferenced assessment. Correlations with Vineland Adaptive Behavior Scales are stronger because it is closer to The oregon Assessment in intent, structure, and procedure. Although it is 
difficult to find instruments that are widely used and have good reliability and validity that are closer in construct to The oregon Assessment, the use of such tools in the congruent research study might have resulted in stronger correlations.

Table 2

Correlations Between Selected Subtests and Total Score's Between The oregon Assessment and The Mccarthy Scales and The Vineland Adaptive Behavior sicales

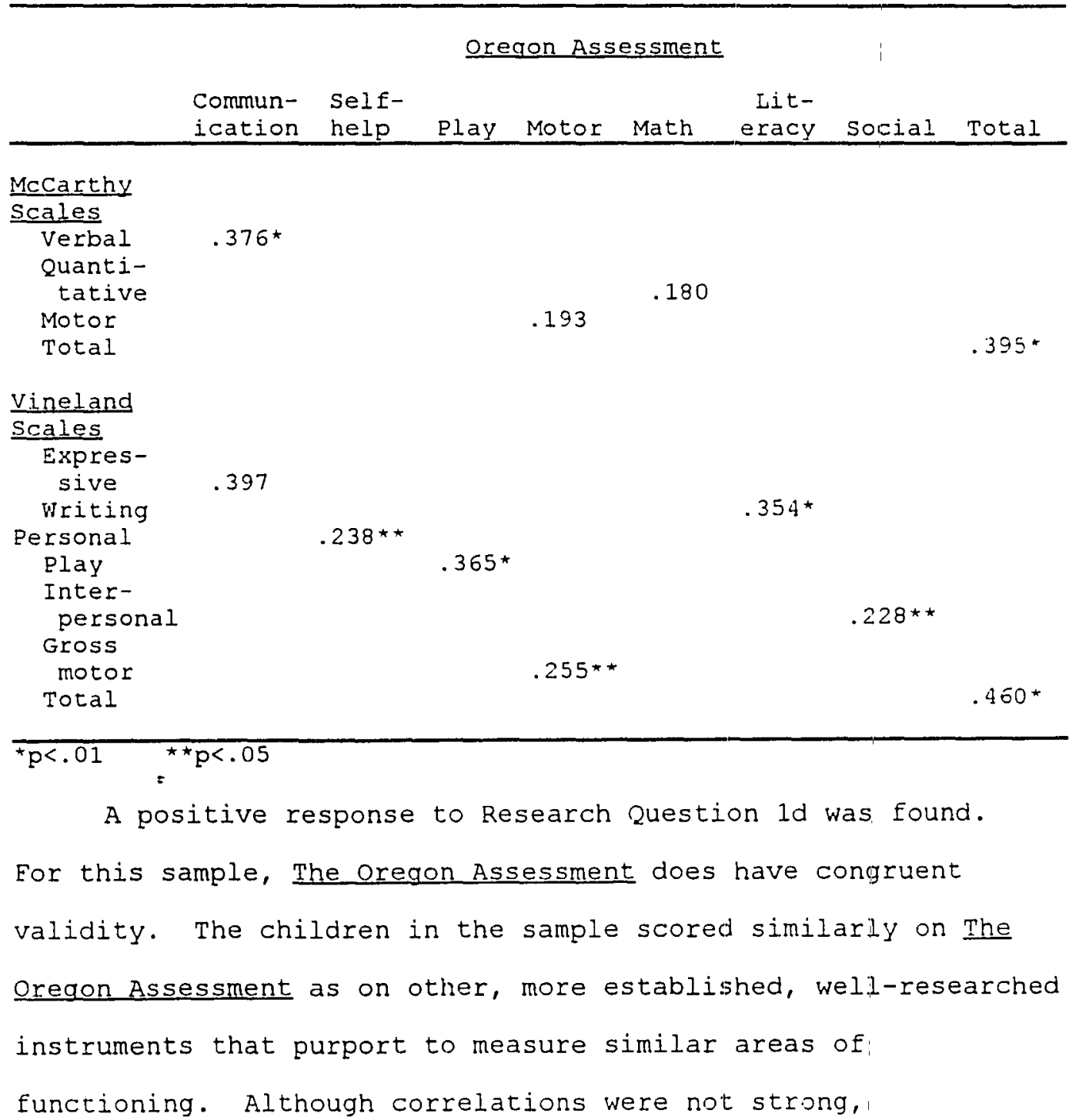


particularly for subtest comparisons, they were significant for some subtest scores and for total scores. The correlation between The Oregon Assessment and Vineland Adaptive Behavior Scales were stronger than between The oregon Assessment and The McCarthy Scales, as The Oregon Assessment and Vineland Adaptive Behavior scales are more similar to each other. The teacher administers those assessments, rather than assessment specialists as with The McCarthy Scales, and they are both weighted towards social and emotional indicators.

Conclusion

Research question 1, Is the Oregon Assessment a valid tool to assess the functioning of young children three to six years of age in preschool classrooms?, can be answered affirmatively for the children and teachers in this sample. Except for unacceptable item validity for one item, and some concern about three other items, the assessment appears to be a valid tool having acceptable levels of curricular, item, construct, and congruent validity. Although generalizing this to other populations must be done carefully due to the geographic and democraphic limitations of the sample. Future revisions of the assessment should eliminate, revise, replace and/or move items $4,8,11,12$.

\section{Reliability}

The following three sections address research question 2: Is The Oregon Assessment a reliable tool to assess the functioning of young children three to six years of age in 
preschool classrooms? Reliability was addressed by determining test-retest, inter-observer, and internal reliability. Internal reliability included four measures: the determination of Chronbach's alpha (or coefficient alpha), the split-half correlation, the spearman-Brown coefficient, and item reliability.

Test-Retest Reliability

Research question $2 a$, Does The Oregon Assessment have test-retest reliability?, was addressed through a study of 80 children (79 usable scores) which included 20 children at each of the four sites. The study found highly significant correlations between all the subtest scores as well as the total score when comparing the first and second administrations of the assessment $(p<.01)$. Self-Help skills revealed the weakest correlation $(r=.479)$ perhaps due to either reinterpretation of the items between the time of the two tests or because these particular behaviors are somewhat variable, subject to changeable emotional states over short periods of time. Although the correlation was significant, it was substantially weaker than the test-retest correlations for other subtest domains. "Containing only three items, including one that was considered problematic for curricular and item validity, the assessment may not construct the self-help domain with enough strength and stability to maintain high integrity across multiple administrations. Strongest correlations were found for the communication sub-test (.938) and for the total score (.916). Communication skills tend to be quite stable over short 
periods of time and there are abundant opportunities to observe communication behaviors in children throughout each day. See Table 3 .

Research question $2 \mathrm{a}$ was answered affirmatively. The

Oregon Assessment has an acceptable degree of test-retest reliability, particularly for the total score and the communication domain. It has stability when given by the same assessor to the same child after a short period of time.

Inter-Observer Reliability

Research question $2 b$ asked, Does The Oregon Assessment have inter-observer reliability? A study of 80 children $(79$ usable scores) in four programs in two northwest states was conducted to ascertain inter-observer reliability. The research found that for this sample, all correlations between administrations of the assessment by different observers at about the same time were highly significant for all subtest scores and for total test scores $(p<.01)$. The weakest correlations were for the self-help (.596) and motor (.515) subtests. The moderate correlation for the self-help domain further indicates that this is a construct that may need strengtheming. That a weaker correlation was found for the test-retest reliability of this domain is an intriguing finding as it was the only domain where this occurred. Possible explanations are discussed in Chapter $V$. The motor subtest had a weaker correlation than the others and had a considerably weaker correlation than for the test-retest study. The strongest correlation was found for the self-concept subtest 
$(.798)$ and for the total score (.852). As self-concept is usually considered a difficult construct to assess because it is viewed as having a greater degree of subjectivity than other areas of development, such as motor or math, it is an important finding that different observers had a higher rate of agreement for this domain than for any other domain. This indicates that self-concept can be a measured with a good degree of stability and consistency. See Table 3 below.

Table 3

Test-Retest Reliability and Inter-Observer Reliability for Oregon Assessment Subtest Scores and Total Scores $(\underline{N}=79)$

\begin{tabular}{lcc}
\hline & $\begin{array}{c}\text { Test-Retest } \\
\text { r Values* }\end{array}$ & $\begin{array}{c}\text { Inter-Observer } \\
\text { Values* }\end{array}$ \\
\hline Play & .324 & .634 \\
Self-Help & .479 & .596 \\
Self-Concept & .824 & .798 \\
Social & .861 & .728 \\
Communication & .938 & .763 \\
Motor & .813 & .515 \\
Dispositions & .850 & .641 \\
Cognitive-Problem & .840 & .753 \\
$\quad$ Solving & .895 & .779 \\
Cognitive-Math & .786 & .757 \\
Cognitive-Literacy & .916 & .852 \\
Total Score & & \\
\hline *pe.0l for all r values & & \\
\hline
\end{tabular}

*p<.01 for all $r$ values

Internal Reliability

Research Question 2c, does The Oregon Assessment have internal reliability?, was determined through four measures: Cr.ronbach's alpha (coefficient alpha), the split-half: correlation, the spearman-Brown coefficient, and item validity. 
The Oregon Assessment appears to have e:cellent internal

reliability as revealed by Chronbach's alpha (coefficient alpha) of .879, a split-half correlation of .984, the Spearman-Brown coefficient of .992, and item reliability scores (Pearson product-moment correlations) for each domain ranging from .627. To .885. See table 4. These are very strong correlations, comparable or better than many other tests on the market (Wiersma \& Jurs, 1990, p. 166). The self-concept domain had the strongest item reliability of any subtest (.885). This provides further evidence that it is a relatively stable and consistent construct, although it has traditionally been considered otherwise.

Table 4

Internal Reliability: Correlations for Item Validity for oregon Assessment subtest Scores, Chronbach's Alpha, The Split-Half Correlation, and The Spearman-Brown Coefficient ( $N=199)$

\begin{tabular}{|c|c|c|}
\hline & \multicolumn{2}{|c|}{$r^{*}$} \\
\hline$=$ & $\begin{array}{l}\text { Play } \\
\text { Self-Help } \\
\text { Self-Concept } \\
\text { Social } \\
\text { Communication } \\
\text { Motor } \\
\text { Dispositions } \\
\text { Problem Solving } \\
\text { Math } \\
\text { Literacy } \\
\text { Chronbach's Alpha } \\
\text { Split-Half } \\
\text { Correlation } \\
\text { Spearman-Brown } \\
\text { Coefficient }\end{array}$ & $\begin{array}{l}.826 \\
.722 \\
.885 \\
.874 \\
.877 \\
.627 \\
.853 \\
.874 \\
.719 \\
.772 \\
.879 \\
.984 \\
.992\end{array}$ \\
\hline
\end{tabular}


The Oregon Assessment has a high level of internal reliability, as determined by strong correlations from three types of split half investigations, Chronbach's alpha, the split-half correlation, and the spearman-Brown coefficient and from item reliability determinations for domains. Research question $2 c$ regarding the internal reliability, was answered in the affirmative.

\section{Utility}

Research question 3 asked, is The Oregon Assessment a useful tool for preschool teachers to effectively assess and plan for children? The answer to the question was determined through the survey responses of 114 users. Ratings revealed that no less than $70.2 \frac{5}{5}$ of respondents agreed or strongly agreed to all statements on the survey. Each of these statements corresponded to a research question related to utility (research questions $3 a-3 n)$. $89.5 \%$ of respondents agreed or strongly agreed that the test includes the most important behaviors to assess (research question 3b, Do teachers and administrators perceive that The Oregon Assessment provides them with important information about children?).

Nearly $88 \%$ of respondents found it to be an effective tool for goal setting for children (research question $3 d$, do teachers and administrators perceive that The oregon Assessment is effective in helping to write goals?). Over $75 \div$ of users responded that it is effective for curriculum planning and 
tracking progress, although more than $23 \%$ felt that it is not (research question $3 \mathrm{e}$, do teachers and administrators perceive that The oregon Assessment is effective for curriculum planning and tracking children's progress?). Apparently taking information from the assessment to create goals for children is facilitated more effectively by the assessment than is the next step in the process, curriculum planning.

Almost $87 \%$ of respondents agreed or strongly agreed that the results accurately reflect the skills, behaviors, and abilities of their children and that The User Manual criteria are clear (research question $3 a$, do teachers and administrators perceive The oregon Assessment to be accurate? and research question 3c, do teachers and administrators perceive the oregon Assessment User Manual to be clear and understandable?). More than $34 \%$ of respondents found the scoring system to be understandable (research question $3 n$, do teachers and administrators find the scoring system to be understandable?). Accuracy and clarity seem to be strengths of the assessment from the perspective of users.

over $75 \%$ of respondents believed it provides a rich and in-depth "picture" of a child (research question 3j, do teachers and administrators perceive that the completed assessment provides a rich and in-depth "picture" of a child?). Just over $20 \%$ of users disagreed or strongly disagreed with this statement. This may be due to a lack of depth in certain domains such as play, self-help, motor, and math, because of few items and some problematic items. 
Although over $70 \frac{3}{3}$ of users felt that it took a reasonable amount of time to administer, more than $27 \%$ of respondents disagreed or strongly disagreed that the test takes a reasonable amount of time to administer (research question $3 \mathrm{~m}$, do teachers and administrators perceive that The oregon Assessment takes a reasonable amount of time to administer?) Additional responses revealed that it takes respondents anywhere from 15 minutes to 20 hours to administer the test with a mean of $13 / 4$ hours and a median of 1 hour (research question $3 k$, "Howl long does it take to complete the assessment on each child?"). This seems fairly reasonable given the observational nature of the assessment. The assessment is administered by respondents over a period of time of between one day and 90 days (research question 31 , over how long a period of time do teachers administer an assessment on a child?). It should be used over time so that the observations are accurate and children are observed lis :acuraily occurring situations (during play and routines). Eighteen days was the mean and five days was the median for the period of time over which a child was typically observed. A two week period seems to be about the right amount of time and this is close to the mean; However, this item received the greatest percentage of negative ratings and therefore the issue of the time it takes to administer the assessment is an area of concern that must be addressed. In addition, while more than $74 \%$ found the assessment to be more useful than other tools that they have used, over 213 disagreed or strongly disagreed with that statement (research question $3 f$, do teachers and administrators 
perceive that The oregon Assessment is more useful than other assessment tools that they have used?). It is likely that these negative ratings are also related to the issue of how much time it takes to administer, as the assessment probably takes more time to administer than other tools, most of which are not observational.

Almost $80 \%$ respondents indicated that parents respond favorably to the assessment and more than $78 \%$ indicated that it helps parents understand and set goals for their children (research question $3 g$, do teachers and administrators perceive that parents respond favorably to The oregon Assessment? and research question $3 \mathrm{~h}$, do teachers and administrators perceive that The Oregon Assessment is useful for helping parents understand their children and set appropriate goals for them?). The results of these items indicate that users view the assessment as a helpful tool in their work with families.

An important goal of the assessment was to create a tool that would be responsive to diversity. Over $76 . \%$ of users indicated that they believe it is responsive to diversity (research question 3i, Do teachers and administrators perceive that The Oregon Assessment is responsive to diversity?). Because this issue has been a difficult issue to deal with in the development of the assessment, and some experts expressed concern on the construct validity research survey about the ability of the assessment to respond to diversity, and over 19 of users gave negative ratings to this statement, it is addressed further in Chapter $V$. 
Table 5

Percentage Ratings of the Utility of

The oregon Assessment $(\mathrm{N}=114)$

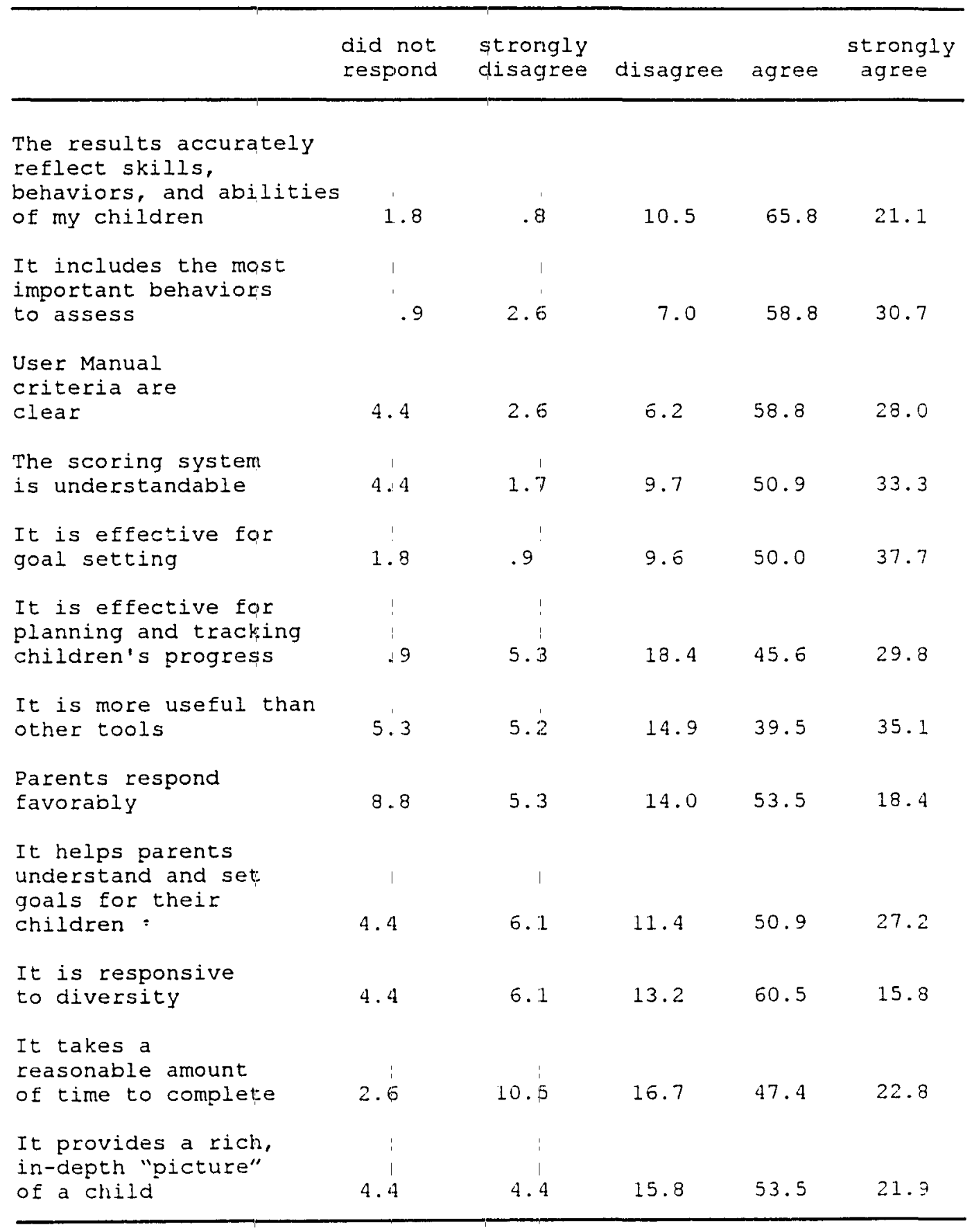


Respondents had the opportunity to provide open-ended written comments at the end of the survey. A typical written response included both critical and positive comments. The following is a summary of those comments.

- There were two generally negative comments and 18 generally positive comments.

- Four respondents indicated that the assessment takes years to get used to before it could be used efficiently and effectively.

- Related to the items themselves, one indicated that they are repetitious, while three respondents said that the assessment does not adequately cover motor skills, two said that it does not adequately cover math skills, two stated that it does not adequately cover behavior problems, and one said that it does not adequately cover play. Four people believed that certain items should be separated into two separate items. Item numbers 8, 20, $23,33,44,60$ were specifically mentioned. One felt that dispositions could be incorporated into various other domains. One indicated that it should include an item related to separation anxiety, one indicated that it is great for its focus on social skills, and one felt it focused too much on social skills.

- One indicated it is not useful with Hispanic children, but one indicated it is helpful with multi-cultural issues.

- Thirteen said that it is too complex or long to use with parents, and five said that parents get too concerned 
about the scores. However there were seven positive comments about it being helpful with working with parents.

- Two respondents indicated it was helpful for writing goals, but one said that it was difficult to use for individualizing.

- Three felt it is too subjective while one described it as accurate.

- Four respondents who didn't answer item \#12 on the survey ("The average amount of time it takes to complete the assessment on each child is...."), said that it varies too much from child to child to be able to respond. Eourteen people indicated that it is too long or takes too much time to administer. A supervisor who responded, wrote that complaints about it being too long are an issue of inadequate training. Two said that they wanted more training.

- Related to scoring, two stated that the scoring should be on a three point system rather than a five point system. One said that scoring the math section is difficult, and four indicated that they just do not like the scoring system.

- While three said that the user manual was helpful, one said that there were still some questions that he or she did not understand.

- That it is a better tool than was used previously was mentioned by four respondents. 
- One expressed concern that children with high skills "top out" too quickly and therefore it is not useful with five year old children.

- A respondent requested development of a curriculum to go along with the assessment, another asked for a screening instrument, and another asked for a summary form that can be passed on to the child's next teacher.

- A series of comments were unintentionally revealing, saying as much about the respondents as about the assessment. One said that children knowing colors was important and it should be included on the assessment, another said that it does not give Kindergarten teachers what they want to know about children, and another that he or she was taught to be objective but then given this subjective assessment to use. A supervisor |commented that many teachers with special education backgrounds had a difficult time with the assessment and this;should be addressed through training. These comments|reveal the gap between long-standing beliefs and practices and the paradigm represented by the assessment.

\section{Eurther Inquiry}

From the survey results and particularly from th e comments, several questions emerged that could provide further insight into the assessment. This insight colild be used to target refinement efforts more effectively. The questions that emerged were: (a) Is there a relationship between users' views or attitudes towards the assessment (negative or positive) and 
the amount of time that people have used the assessment or the amount of training they received?, (b) Is there a relationship between perceptions about the time it takes to administer the assessment (reasonable or unreasonable) and either the amount of time used or the amount of training received?, and (c) Is there a relationship between users' attitudes towards the assessment and their perceptions about how long it takes to administer? To answer these questions, scores from three items on the questionnaire were aggregated into one new variable called attitude. These items were: (a) Does the assessment include the most important behaviors to assess in children?, (b) Is the assessment an effective tool for me to plan individualized curriculum and track children's progress?, and (c) Does the completed assessment provide a rich and in-depth "picture" of a child? The scores on this attitude variable were then dichotomized into negative attitude (score of 1 or 2 ) or positive attitude (score of 3 or 4 ). Scores on the "time used" variable were dichotomized into shorter time used (less than 2 years) or longer time used (more than 2 years), as 2.1 was the mean number of years used. Scores on the amount of training received were dichotomized into shorter time trained (4 hours or less) or longer time trained ( 5 hours or more) as the mean was about 4-5 hours. Scores on the variable of perceptions of how long it takes to administer the assessment were dichotomized into unreasonable ( 1 or 2 ) or reasonable ( 3 or 4 ). The author hypothesized that there would be a significant, positive relationship between attitude and amount of time used, between 
attitude and amount of training received, between perceptions of the time it takes to administer and amount of time used, between perceptions of the time it takes to administer and amount of training received, and between attitude and perceptions of the time it takes to administer. Any case where there was no response to any of these items was deleted from the set. This left 85 cases from the original 114. Five multi-way, chi square tabulations were then run to determine the strength of the relationships.

As illustrated in Table 6 , there was no significant relationship between attitude toward the assessment and the amount of time used nor the amount of training received. Neither was there a significant relationship between perceptions of the time it takes to administer and the amount of training received. However, there was a significant, positive relationship between attitude towards the assessment and perceptions of the time it takes to administer $(p<.05)$ and between perceptions of the time it takes to administer and the amount of time used $(p<.01)$. Users who have used the assessment for two years or more view it as taking a reasonable amount of time to administer, while those who have used it fewer than 2 years view it as taking an unreasonable amount of time to administer. This may be an indication of the learning curve needed to use the assessment efficiently. Providing more training and greater on-going support may decrease the time it: takes to find the assessment reasonable to use in terms of time. Although there is a significant relationship between attitude 
towards the assessment and perceptions of the time it takes to administer, it is not possible to ascertain in which direction the influence runs. Do users who believe it takes a reasonable amount of time to administer view it favorably because of this factor or do users who view it favorably tend to believe it takes a reasonable amount of time to administer? In either case the issue of the time it takes to administer needs to be looked at closely because attitudes towards the assessment are closely associated with perceptions of the time it takes to administer.

Table 6

Relationships Among Several Utility Variables and and Several User Demographic Variables ( $\underline{\mathrm{N}}=85$ )

\begin{tabular}{lccc}
\hline & $\begin{array}{l}\text { Pearson Chi } \\
\text { Square Value }\end{array}$ & DF & $\begin{array}{c}\text { Prob- } \\
\text { ability }\end{array}$ \\
\hline $\begin{array}{l}\text { Attitude x Amount of } \\
\text { Time Used }\end{array}$ & .256 & 1 & .613 \\
$\begin{array}{l}\text { Attitude x Amount of } \\
\text { Training }\end{array}$ & .926 & 1 & .336 \\
$\begin{array}{l}\text { Perceptions of Time } \\
\text { it Takes to Administer } \\
\text { x Amount of Training }\end{array}$ & .689 & 1 & .406 \\
$\begin{array}{l}\text { Attitude x Perceptions } \\
\text { of Time it Takes } \\
\text { to Administer }\end{array}$ & & & \\
$\begin{array}{l}\text { Perceptions of Time It } \\
\text { Takes to Administer } x\end{array}$ & & 1 & .013 \\
Amount of Time Used & 7.937 & 1 & .005 \\
\hline
\end{tabular}

The utility or usefulness of The oregon Assessment was determined through a questionnaire completed by 114 users. 
Research question 3, Is The Oregon Assessment a useful tool for preschool teachers to effectively assess and plan for children? -- and the various sub-questions $(3 a-3 n)$-- were answered affirmatively. Although there was some concern about the time it takes to administer, its effectiveness in helping with planning and tracking children's progress, it's ability to provide a rich, in-depth "picture" of a child, and it being more useful than other tools respondents have used, the assessment was found to have an acceptable level of utility. Users particularly found it to be accurate, clear, helpful with goal setting, and inclusive of the most important skills or abilities to assess. Further inquiry found that respondents who used the assessment for two years or more perceived that it takes a reasonable amount of time to administer, while those using it less than two years perceived it takes a unreasonable amount of time to administer. There was a very close relationship between users' attitudes towards the assessment and their perceptions of whether or not it takes a reasonable amount of time to administer.

\section{Conclusion}

The results of the research determined that The oregon Assessment has a high degree of curricula validity on all items, domains, and all domains taken together as a whole, although four of the 60 items had lower validity ratings than the others. These four items also had the lowest item validity ratings and one of them, item \#4 ("Puts materials in proper place when 
finished") received an unacceptable item validity rating. These items will need to be addressed in the refinement of the assessment. There were numerous comments on the survey related to curricular validity but none related to item validity. The assessment was found to have a high degree of construct validity as determined by one question on the survey and through the process of the assessment's development. There were three comments related to construct validity, two of them concerned its responsiveness to diversity. It was found to have moderate but significant congruent validity with total scores on the McCarthy Scales and the Vineland Adaptive Behavior Scales.

Test-retest, inter-observer, and internal reliability were found to be strong. Correlations between total test scores and subtest scores were significant in both the test-retest study and the inter-observer study $(p<.01)$. Chronbach's alpha was .879, the split-half correlation was .984, and the SpearmanBrown coefficient was .992, indicating a high degree of internal consistency.

The utility study determined that the assessment is generally a useful and effective tool, particularly in terms of accuracy, clarity, help with goal setting, and the inclusion of important skills and behaviors to assess. Although there was concern about it being too long, users who have used the assessment for two years or more found it to take a reasonable amount of time to administer. Those who like the assessment find it takes a reasonable amount of time to administer and 
those who think it takes a reasonable amount of time to administer tend to like the assessment.

The results indicate that the general research question, "Is The Oregon Assessment for 3-5 Year Olds in Developmentally Appropriate Classrooms a valid, reliable, and useful assessment tool?", can be answered in the affirmative. It appears to be an effective instrument for its intended purposes of assessing children's functioning, tracking progress, and assisting teachers with individualized curriculum planning, at least for the population used in the sample. It also appears to adhere to the guidelines of appropriate and equitable assessment for young children. In addition it should serve well as a research tool for program evaluation research, particularly for programs like Head start that focus on the development of children's social competence. 
CHAPTER V

\section{DISCUSSION AND RECOMMENDATIONS}

This chapter presents a discussion of the results of the research and recommendations for further research and refinement of The oregon Assessment. A summary of the results for each study related to each main research question will be presented followed by a discussion of the implications and concerns raised by the results. These are followed by recommendations. The studies discussed include curricular, item, construct, and congruent validity; test-retest, inter-observer, and internal reliability; and utility of The oregon Assessment.

\section{Curricular Validity}

All items individually, each domain, and all domains taken together as a whole were found to have an acceptable or high degree of curricular validity based on the survey results from 15 early childhood education experts. Four items are of some concern, however, as they have mean scores ranging from 3.0 to 3.2 and median scores of 3 . These are item 4 , "Puts material in proper place when finished"; item \#8, "Cares for personal safety -- uses seat belt, is not reckless on playground, in classroom, and on field trips"; item \#11, "Makes eye contact (providing this is a cultural norm)"; and item \#12, "Shows appropriate caution with strangers". Although there were no 
written comments specifically related to these items, it appears that the experts believe they are not istrongly related to the constructs of developmentally appropriate practices. Perhaps they are too focused on specific skills that are context and culture bound or have too high an expectation for a preschool child. The construct of developmentally appropriate practices is based on age and individual responsiveness, building on children's strengths and reducing stress (among other principles). If the focus of observing children's behaviors is on narrow skills, then individual responsiveness is reduced, compensatory strengths tend to be ignored if the skills are weak, and stress on children can increase. Assessment requires specificity. Decisions must be made about which particular behaviors, skills, and knowledge are most important. This is likely to reduce flexibility and responsiveness, whereas the developmentally appropriate practices guidelines were developed, in part, to promote more flexibility and responsiveness in early childhood programs (Bredekamp, 1987). | Perhaps the respondent who expressed concern that developmentally appropriate assessment may be impossible, was addressing this issue.

In addition, certain items such as \#12, "Shows appropriate caution with strangers", may have been rated lower on curricular validity because they cannot be easily observed in the classroom. They are too far removed from typical curricular practices and routines. Because the four items of concern were also identified as problematic items from the item validity 
research, they are discussed in more specific detail in the next section which discusses item validity.

In a revised version of The oregon Assessment it may be necessary to replace, remove, or revise these four items. Because they were developed in a collaborative process with practitioners, it would be a good strategy to convene several focus groups comprised of experts, practitioners, users, and non-users to give feedback about whether to eliminate these items, revise them, or move them. If they are to be revised, the focus groups could recommend how they should be changed. Based on the comments made by the 15 experts, The User Manual will be revised to fix the typos and unclear statements they found. It is difficult to see any strong trends from the comments as some recommended more items with greater difficulty while others were concerned that it was already too long and too difficult for many teachers to administer. (As discussed in the utility section of this chapter, there is some corroboration from users that the assessment is too long but little indication that it is too difficult). One response to this would be to develop several different versions of various lengths, format, and detail from which practitioners could choose. The problem with this approach is that all versions would need to undergo validity and reliability research, requiring a great deal of time and resources. 
Item Validity

Eifty-nine of the 60 items on the assessment were found to have acceptable or high levels of item validity (they are important indicators of their respective domains), based on the survey results from 15 early childhood education experts. Mean scores for these 59 items were 3.0 or greater and 56 of the 60 items had median scores of 4 .

The four items of concern discussed in the curricular validity section also received some of the lowest item validity scores. Item \#4, "Puts materials in proper place when finished" received an unacceptable score for item validity; it was the only item that received an unacceptable score for either curricula or item validity. This is clearly an item that either should be eliminated, altered greatly, and/or moved to another section. It perhaps fits better under self-help skills and might have received an acceptable score under that domain. With only five items in the play domain, there could easily be a more apropos item related to play skills or behaviors rather than "clean-up" behaviors. Some ideas include: (a) uses imagination during play, (b) pretends that objects represent other objects, or (c) cain enter play with others or another effectively. The importance of eliminating, revising, and/or moving the other three items $(8,11$, and 12) is underscored by the results of this item validity research. Item \#8, "Cares for personal safety - uses seat belt, is not reckless on playground, in classroom, and on field trips" may be viewed by respondents as a high level skill that is unrealistic to expect a preschool age 
child to do. It could easily replaced by another self-help item, as item \#44, "Enjoys taking risks while maintaining safety of self and others", covers similar behaviors. In addition, item \#44 received higher ratings with a mean of 3.7 and median of 4 for both curricula validity and item validity. Other selfhelp items to consider include but are not limited to: (a) eats an appropriate amount of food for age and weight and enjoys most foods, (b) uses toilet appropriately -- flushes and washes hands, and (C) can take off and put on shoes and other clothes. Item \#11, "Makes eye contact (providing this is a cultural norm)" and item \#12, "Shows appropriate caution with strangers" could be easily eliminated as there are 12 self-concept items. Reducing the number of items to 10 in this domain would make it more in proportion with most of the other domains in terms of length (although dispositions has 13 items), while still providing a comprehensive picture of the child's functioning in this area of development. These recommendations, related to the four items of concern, should be reviewed by the focus groups described previously. Any new items would need to be validated using a similar method to the curricular and item validity studies utilizing survey data obtained from experts.

$$
\text { Construct validity }
$$

The assessment was found to have strong construct validity based on the collaborative process with practitioners used to develop it and the positive ratings from experts for this item on the survey. Nevertheless several issues emerged from the 
research related to construct validity. In this section three issues will be discussed: (a) the comments made by respondents, (b) portfolio assessment as an important assessment construct, and (c) different ways that the assessment could be conceived and organized.

\section{Comments}

Although the 15 expert respondents clearly gave the assessment a highly favorable rating in response to the survey item related to construct validity, the three comments specifically about construct validity need to be discussed. Two of these three comments were related to cultural issues (or responsiveness to diversity). One of these stated that some items value behaviors that may not reflect cultures of family styles. The other said that because of its focus on dispositions and social/emotional functioning it is too valueladen and therefore culture-bound. However this same respondent indicated that he or she supports the values represented by the assessment and has not resolved the dilemma of assessing across cultures. The only other comment related to construct validity was from one respondent who felt that cognitive math items are problematic because they would likely lead teachers to do direct testing rather than observation.

The User Manual and training sessions address the issue of culture and diversity by explaining the concept of bi-cultural (or multi-cultural) competence. A review of the literature revealed that this is one important way to deal with the dilemma posed by one expert about assessing across cultures (Albert \& 
Triandis, 1991; De Leon, 1990; Saracho \& Hancock, 1983). It is not possible to be culturally neutral or culture blind on any assessment, therefore it is best to make cultural issues explicit and support the child's competencies in the culture(s) reflected in the school as well as the culture(s) in the home (see pages 42-48 of The User Manual, Appendix C, for further explanation). Two of the expert respondents apparently did not read this or were not satisfied by it. The issue of testing and culture is complex and emotionally volatile. Even when addressed by the manual and during training, the possibility remains that aspects of a child's home culture will be diminished by educators because some assessment items have a different value set. There are no easy or quick solutions to this problem. The process of sensitizing teachers to the issues of culture and helping them support each child's home culture while still assisting children to be competent in the school culture is an on-going, difficult task of pre- and in-service teacher development. The issue needs to be addressed in every course and every training session. For the oregon Assessment, an attempt was made to deal with the issue directly, explicitly, and in the most effective way known to the author at the time. It is only when educators confront the issue and develop skills and strategies to value diversity across all aspects of teaching and learning -- curriculum, materials, language use, school structure, parent relations, etc. -- that educators will feel comfortable and effectively deal with the issue as part of the 
assessment process (Bowman, 1992; Derman-Sparks, 1992; LadsonBillings, 1992).

The math items on the assessment do tend to be the most specific and most difficult to observe naturalistically from spontaneous behavior. For that reason, the directions on the assessment say to observe math skills, "When (the child is) playing with objects in the environment such as blocks, pegs, dishes, etc." (p. 5). The User Manual also gives examples of ways to observe the skills during play or routines (p. 23-24). Even with this guidance, it may be difficult to observe a child seriating during play, for example. To address this problem, during training on using the assessment, users are given ideas for specific small group math games so that these skills can emerge and are more likely to be seen. Although they are teacher structured activities, the skills would be observed during the course of play rather than through direct questioning of the child in a contrived situation. These small group math game ideas need to be included in a revision of The User Manual to provide further assistance to teachers and reduce the likelihood of direct assessment.

\section{Portfolia Assessment}

Portfolio assessment, or the compilation of materials and documents that show a student's best work and progress over time, is a construct of assessment that is very popular with practitioners and highly touted by many educators (Gifford \& O'Connor, 1992; Grace \& Stores, 1992; Gullo, 1994; Wortham, 1990). It is not a construct used in the development of the 
oregon Assessment. Although the assessment achieved a high degree of curricular validity and there were few calls from users or experts for a portfolio process as part of the assessment, providing such a process would give users more options based on more constructs. This potentially increases its construct validity and helps to assure its future viability.

A process for using the assessment as a portfolio assessment could be easily developed. Such a process should be included in The User Manual and developed as a separate document. As all items are observable, the development of a portfolio materials in conjunction with the assessment is a natural extension of many of the assessment items. The observations related to specific items could be documented via audio or video tape, as well as through written anecdotes, if taping is not possible or efficient. Many of the items, particular those in the literacy and math domains and creative art in the dispositions domain, could be documented through the collection of materials produced by the child. The portfolio items could be coded with the assessment item number with which it corresponds and kept in chronological order. Eor example, a child's oregon Assessment might be kept in a small box which also contains various dividers or file folders. Each file folder is labeled with an item number from the assessment and kept in numerical order. The folder labeled, "Item \#33 -Initiates and sustains conversations", contains an audio tape with recordings of the child initiating and sustaining three conversations at the lunch table. The first conversation 
occurred in October, the second in February, and the third in May. The increasing number and intensity of interactions, length of sentences, and responsiveness and persistence across time is easy to hear and explicitly documented on the tape. Folders for other items are included in a similar manner although others might contain video tapes, artwork, writing samples, etc., depending on the item. Next to each item on the assessment itself is a written remark indicating if a portfolio file exists for that item and the media that was used for the portfolio evidence.

\section{Alternative Constructs}

The Oregon Assessment was developed based on the assumption that using the typical developmental domains of language, motor, cognition, social, self-help, etc. was the only valid way to view children's development and therefore the only way to construct an observational assessment. Although a few non-traditional domains were added (play and dispositions), the use of typical domains of development remained largely intact. Although the assessment achieved a high level of construct validity, it would be interesting and useful to determine its construct validity based on an alternative construct of developmental domains because the organization and categorization of an assessment reflects a particular construct of development and communicates implicit but powerful messages about how development is viewed and what is important for children. 
Several recent articles and publications have presented a different construct for viewing children's development than through the usual developmental domains (Griffin, 1994, 1995; Bredekamp \& Rosegrant, 1992). Griffin (1994, 1995) presents a way to organize development around different domains. These include confidence, curiosity, intentionality, self-control, relatedness, capacity to communicate, and cooperativeness (p. 67). Most of these can be viewed as dispositions. As the dispositions domain is the largest one (contains the most items) on The oregon Assessment, restructuring it around these or similar domains with the various abilities and behaviors (motor functioning, math skills, literacy skills, etc.) keyed to each dispositional domain, makes a great deal of sense. It would also send a powerful message to users about the importance of dispositions in teaching and learning.

Bredekamp and Rosegrant (1992) present a similar construct of development based around certain developmental dispositions. In their schema these are persistence, focused participation, hypothesis testing, risk taking, and self-regulation (p. 75-76). Here too, particular behaviors for test items related to a various functions -- language, motor, social, etc. -- could be included under each disposition. If a language item such as item \#28, "Listens to and contributes to group discussions" was one of a number of items under a category called "focused participation", and a different language item such as \#31, "Communicates so s/he is understood by others" was one of a number of items under the "persistence" domain, the user would 
get the message that the ability to use language to sustain effort and engage meaningfully with others are the important issues, not the use of language per se.

This is an intriguing idea that could greatly assist in the effort to change teaching practices. It may be that some of the dissatisfaction expressed by some users stems from the fact that the assessment is organized much as other assessments but has untypical content and represents a different paradigm than most other assessments. This may confuse some users, as send it sends them a mixed message. Many early childhood educators view development and their role in promoting development from a primarily individualistic and cognitive perspective, however, The Oregon Assessment represents a developmental constrict based on interactionalism, dispositions, multiple intelligences, etc. If the assessment were constructed differently, it may signal users, in an indirect but powerful way, that they will be assessing children differently than usual. However, it could also result in alienating users further because it may be too different from what they have used before and what they have been taught. It would be a worthwhile endeavor to field-test a version of the assessment constructed around different domains of development and gather some initial data about its construct validity. It may turn out to have a lower level of construct validity than an assessment constructed around the typical domains of development such as The oregon Assessment, because it would be too different from users' current conceptualizations of development and assessment. One the other hand, it may have 
greater construct validity because it more fully integrates the paradigm of development represented by the assessment. A future research project on this order could provide important insights into these issues.

\section{Congruent validity}

Moderate but significant correlations were found for total scores and for some of the subtest scores of similar domains between The oregon Assessment and The McCarthy Scales for Children and The Vineland Scales Adaptive Behavior Scales,. These findings were based on a sample of 200 children (199 usable scores) from four programs in two northwest states. As discussed in Chapter III, the sample is fairly representative of the northwest but not the U.S. in general. A major shortcoming of this research study is the lack of generalizability of the results because of the sample used. Euture research on the assessment should use a sample that represents the general U.S. population and should include a larger sample size.

The moderate but significant correlations between The Oregon Assessment and The McCarthy Scales and The Vineland Scales were expected as The oregon Assessment measures somewhat different aspects of developmental domains (dispositions more than skills) than either of the other assessments. The methods used to score the assessments also differ. The McCarthy scales is scored by direct testing (usually not done by the teacher) of each child individually in a different setting than the classroom and The Vineland Scales is scored by the teacher from 
memory at single sitting. The Vineland scales correlation may be stronger than The Mccarthy Scales correlation because the Vineland Scales and The Oregon Assessment were scored by the classroom teacher and The McCarthy Scales by a professional researcher who was a stranger to the child.

A very high correlation between a new assessment and an established assessment is not necessarily desirable because they would then essentially measure the same things. The new instrument would not contribute something new to the field of assessment, unless it was easier and quicker to use or provided some other advantage. A moderate correlation is more desirable as children who tend to score low (or high) on one assessment should also score low (or high) on another assessment that measures similar attributes.

The lower correlation between subtests of the same domains (i.e.; motor, math, social) than the correlations between total scores of The Oregon Assessment and either of the other assessments may reflect the difference in the purpose and methodology among the assessments. Perhaps total scores represent more general tendencies and there is more congruence between the assessments in general (low scores equate with low scores and high scores with high scores) then in the specifics. For example, the correlation between the math subtest score on The oregon Assessment and the quantitative subtest score on The McCarthy Scales is .180 (not significant). Math is assessed through naturalistic observation of children in play situations and it includes a broad range of skills rather than only 
quantifying (i.e.; seriating, patterning) on the oregon

Assessment. Math skills, involving only quantifying, are assessed on The Mccarthy scales by directly asking a child to count objects (one inch cubes). What is assessed and how the data are collected is very different even though they appear to be very similar domains. These differences would logically result in quite different scores.

The congruent validity results are the weakest results of any of the studies done on the assessment. Future research on the assessment should include a congruent validity study using measures more similar to The oregon Assessment in process and intent. This is not a simple task because there appear to be few such measures that have been in existence long enough to have a good track record in the research literature and also have good results from their own rigorous validity, reliability and utility studies.

\section{Reliability}

The three reliability studies, test-retest, interobserver, and internal reliability (four measures of internal reliability included Chronbach's alpha, the split-half correlation, the spearman-Brown coefficient, and item reliability) had the strongest results of the various studies conducted in this research project. All results were highly significant (p.<.01) and Chronbach's alpha (coefficient alpha) was .879. The studies used a sample of 200 children 199 usable scores) from four programs in two northwest states. As 
discussed in Chapter III, the sample is fairly representative of the northwest but not the U.S. in general. A major shortcoming of the reliability research study is the lack of generalizability of the results because of the sample used. Euture research on the assessment should use a sample that represents the general U.S. population and should include a larger sample size. The following sections discuss the results of the test-retest and inter-observer reliability studies and the internal reliability study.

Test-Retest and Inter-Observer Reliability

The test-retest reliability correlations were stronger then the inter-observer correlations for the total score and all domains except one, self-help. It is to be expected that testretest correlations would be stronger than inter-observer correlations because a single user is more likely to be consistent in his or her interpretation of test items and judgements of a child than different assessors when compared to each other.

Reliabilitv Issues Regarding the Self-Help Domain Subtest. Eor the self-help domain, the test-retest correlation was .479 and the inter-observer correlation was .596 . This was the weakest correlation of any domain for test-retest validity with all others ranging from .786 (cognitive-literacy) to .939 (communication). It was the second weakest correlation of any donain for inter-observer reliability (motor had .515) with the others ranging from .641 (dispositions) to .798 (self-concept). This indicates that for this domain only, the same tester 
administering the test twice to the same child varied in his or her interpretation of the child's functioning on this domain more than different testers who tested the same child at about the same time. This anomaly may result from several factors. The self-help domain has only three items, one of which was considered to be problematic from the curricula and item validity studies. Perhaps this does not give the domain enough strength to hold its construct with a very high degree of consistency over time. It also may be susceptible to greater variance over time than other domains because it is contingent on emotional states that in young children are volatile, changing easily and quickly over short periods of time. A young child may be willing to take care of his or her own needs one day but not the next and it may be difficult to determine what constitutes typical behavior. Another possible explanation is that the items are not as clear as they could be and received slightly different interpretations at different times. There is not a strong case for this explanation as it would appear that lack of clarity would cause more variance between different users at least as much if not more than with the same user. If the survey asked for feedback on the technical quality of the items there might have been some information about this regarding the self-help items.

Reliability Issues Regarding the Motor Domain. For the motor domain, the test-retest correlation was much higher (.813) than the inter-observer correlation (.515). This indicates that different testers varied more on the interpretation of a child's 
functioning in this area than the same tester who tested the child twice, to a great extent. As with the self-help domain, the motor domain has only three items. The weakness of construct for this domain (as it is based on only three items) seems to reveal itself more in somewhat differing interpretations between assessors rather than from the same assessor (as with self-help). Motor skills tend to develop slowly and gradualiy and are not as subject to dramatic changes over short periods of time, as they tend not to be as directly influenced by volatile emotional states as self-help skills. Eor this reason poor technical quality of the items and/or directions in The User Manual are more suspect with this domain than self-help. A single user is more likely to interpret unclear items a particular way and consistently interpret them that way (keeping test-retest correlations high), while multiple users are more likely to interpret unclear items differently from each other (reducing inter-observer reliability).

For all other domains, there were not great discrepancies between test-rest correlations and inter-observer correlations. For the domains discussed, motor and self-help, all correlations, regardless of the differences between the two studies on the same domains, were highly significant $(p<.01)$. Strong Reliability: Communication and Self-Concept. The strongest correlation for test-retest reliability was the communication domain. Unlike self-help skills, communication skills tend to be relatively stable over the course of a short period of time. Growth in communication skills are usually 
gradually and are not typically subject to volatile emotional states. In addition, the typical early childhood classroom provides teachers with numerous opportunities to observe communication behaviors as most children interact with each other and with the adults often and regularly.

The strongest correlation in the inter-observer reliability study was found for the self-concept domain. As discussed in the next section, internal reliability, selfconcept appears to be a stable and consistent measure on this assessment. The high level of stability between assessors on this domain indicates that it can be accurately assessed and there is al high degree of common understanding of how to assess self-concept behaviors. This is an important finding of the research project, as this domain has been traditionally viewed as being too subjective to measure accurately. That it achieved a higher level of inter-observer reliability than any other domain, including those traditionally considered more objective (motor, math, communication) is an important finding.

\section{Internal Reliability}

The most compelling results of all the studies done on The Oregon Assessment, were from the data obtained for internal reliability. The very strong correlations for Chronbach's alpha (.879), the split-half correlation, (.984), the Spearman-Brown coefficient (.992), and item reliability indicate excellent internal consistency for the assessment. Three of these measures determine internal consistency through a split-test procedure, while item reliability is determined by obtaining a 
Pearson product-moment coefficient for the domains (and items) as they correlate to total scores. This is a measure of internal reliability for individual items or domains rather than the test as whole. These measures are important indicators of its consistency, crucial to determining the efficacy of any assessment (Ebel \& Erisbie, 1991; Wiersma \& Jurs, 1990). A major positive achievement of the development of the assessment and the research was obtaining a very high level of internal reliability for each domain and for the test as a whole.

These findings are particularly important because tests of "softer" characteristics or behaviors tend to have weaker internal reliability ratings than tests of "harder" ones (Wiersma \& Jurs, 1990). A key goal for the development of The oregon Assessment, was to create an instrument that could accurately measure attributes that have been traditionally viewed as difficult to measure accurately because they are "subjective" or "soft", such as social functioning, selfconcept, and dispositions (similar to attitudes). For this assessment, these domains tended to have stronger internal reliability ratings ( self-concept $=.885$, social $=.874$ ) then those traditionally considered "objective" or "hard" such as math (.718) and motor (.627). This may be because the domains with stronger reliability ratings tended to have more items and because many of the early childhood teachers in this study are now more knowledgeable and keen observers of children's selfconcept development and social skills. They are now making these domains their primary educational focus. As the test as a 
whole is weighted towards these "softer" domains, obtaining a Spearman-Brown reliability rating of .992 is important because this compares favorably to any assessment on the market. The Stanford Early School Achievement Test reliability is $.76-.96$ (the variation is due to different reliability research studies), The Piers-Harris Children's Self-Concept Scale reliability is .90 and The Iowa Tests of Educational Development reliability is .90 (Wiersma \& Jurs, 1990, p. 166).

If, in response to users, a shorter version of the assessment is developed (as will be discussed later in this chapter), the excellent internal reliability may be diminished. As Wiersma and Jurs (1990) indicate, "all other factors being equal, the longer the test the greater the reliability" (p. 159). Only through conducting a similar research study again can it be determined if a shorter version will be have lower or unacceptably low internal reliability.

\section{Utility}

The assessment obtained an acceptable degree of utility, based on the survey results from 114 users throughout the U.S. More than two thirds of respondents gave a positive evaluation ( 3 or 4) to each issue or concern raised on the survey regarding usability. On several key issues, the inclusion of the most important skills or abilities to assess, the effectiveness of the assessment for goal setting, the clarity of criteria for the items, and the accuracy of the assessment, $87 \div$ to 89.5 : of respondents gave favorable evaluations ( 3 or 4 ). 
There is concern, however, regarding the survey items that were rated negatively (1 or 2) by more than $20 \%$ of respondents. These are: (a) it is effective for planning and tracking children's progress, (b) it provides a rich, in-depth "picture" of a child, (c) it is more useful than other tools and, (d) it takes a reasonable amount of time to complete. This last item received the most negative ratings of any item with $27.2 \%$ of respondents scoring it 1 or 2 . It also had the highest percentage of ratings of 1 (10.5\%) -- the most negative response possible -- of any of the survey items. The following sections provide a discussion of possible reasons for these negative ratings. Although all these items were rated much more positively by more users than negatively, they will be discussed because it is important to subject an assessment tool to rigorous scrutiny due to its potential impact on children, teachers, curricula, and educational environments.

Is It Helpful With Planning and Tracking?

If used only to assess the current functioning of children, the assessment's main purpose -- to assist in individualized curriculum planning -- is not achieved. Its usefulness for tracking children's progress, an important secondary purpose, is also lost. To achieve it's full purpose, the assessment must be used to set goals for children, plan curriculum to incorporate those goals, and track children's progress.

There was only one respondent comment on the surveys related to the issue of planning and tracking and it was too 
general to provide insight into this issue. The survey, unfortunately, did not determine how many respondents use the planning forms or follow the color-coded progress tracking system suggested on each page of the assessment. Nor did it include an item for determining how many users received specific training on planning and tracking progress or continue to receive on-going support for this. It may be that many of the users who gave negative ratings to this survey item did not receive training on this, do not have access to the planning forms, or are not encouraged or guided to use it for these purposes. If the item had been divided into two separate items, one related to planning and one related to tracking progress, more specific and useful information would have been obtained. It may be widely used for planning but not tracking children's progress, or vice versa. Nevertheless this is clearly an issue that needs to be addressed.

One of the highest rated items was, "it is effective for helping me write goals for children". This seems to indicate that users are easily taking the next step in the assessment process of using the information gathered on the assessment to write goals for children. The wording of items on the assessment was carefully constructed for that purpose. By changing only a word or two an item can be written as a goal. For example, item number 22, "Listens to entire story during storytime" can become a goal when worded as, "Jasmine will listen to the entire story during storytime" or "Mason will listen to half the story during storytime". It appears, 
however, that it is more difficult for teachers to take the next steps after goal setting, which are to address the goals through specific curriculum plans and record and track children's progress towards the goals. Teachers may not have access to the forms that facilitate this and/or have not received training and support specifically on this. Training content and The User Manual will need to be revised to better promote the practices of using the assessment for planning and for tracking progress. Another strategy to promote the use of the assessment for individualized planning would be to include the planning forms with each assessment tool. Currently it is provided separately, usually as part of a hand-out package at training sessions.

Does It Provide a Rich, In-depth "Picture" of a Child?

Just over $20 \%$ of respondents felt that it does not provide a rich, in-depth "picture" of a child. Some of the comments from the survey provide some insight into this issue. A number of users indicated that the assessment does not adequately cover motor skills, math skills, behavior problems, separation anxiety, and play. Additionally four users indicated that certain items $(8,20,23,33,44$, and 60 specifically) should be divided into two separate items. Although providing more items would give a richer, more in-depth "picture", many more users (and one expert) expressed concern about its length and complexity. Although most users have probably used assessments that contain more items (The Vineland Scales contain 144 items), providing more depth, they were quicker to use because items were directly assessed or assessed from memory. Because 
observational, contextual assessments take longer -- behaviors and skills are seen when they occur naturally in the environment -- the number of items on The Oregon Assessment was kept relatively small to make it possible to complete the assessment in a reasonable amount of time. However, if accuracy is lost when assessments are decontextualized, then the "depth" they can provide may not be worth the sacrifice. Accuracy was identified as a strength of The Oregon Assessment by users $186.9 \%$ agreed or strongly agreed with the item, "The results accurately reflect skills, behaviors, and abilities of my children"). Herein lies a great dilemma of developmentally appropriate assessment: lack of accuracy can ultimately be harmful to children (resulting in misguided interventions), but obtaining accuracy is a complex, time consuming process. Rather than skirting this dilemma, the author attempted to address it directly by trying to develop a tool that would balance the need for accuracy with functionality (in terms of the time it takes to administer) by delimiting items while still providing adequate depth. Critics of current assessment practices and practitioners in the field made it clear to the author that sacrificing accuracy is no longer an acceptable option. Refining the instrument by replacing problematic items and developing various versions of the assessment could further expedite the goal of adequately addressing the dilemma.

Is It More Useful Than other Tools?

It is likely that the issue of the assessment rated as not being more useful than other tools used by about 20: of 
respondents, is also related to the issue of the time it takes to administer. If it takes longer to administer than previous tools used, as is probable because it is observational, a riumber of users would find it less useful. The issue of time is a major concern for most early childhood teachers who are asked to do much for little compensation and who often work longer hours and more days per year than other teachers (Helburn, Culkin, Morris, Moran, Howes, Phillipsen, Bryant, Clifford, Cryer, Peisner-Feinberg, Burchinal, Kagan, \& Rustici, 1995). The length of time it takes to administer the assessment emerged as the salient concern from the survey items and from the comments made by respondents. Chi square analysis of aggregated and dichotomized variables from the survey revealed a significant, positive relationship between users' general attitudes towards the assessment (negative or positive) and their perceptions of whether the assessment takes a reasonable or unreasonable amount of time to administer. The only other area where there appeared to be significant concern from the comments was related to its use with parents. Many of these comments indicated that it was too long to use effectively with parents, bringing it back to: the length issue. There was no real consensus about this, however, as seven respondents indicated that the assessment was particularly helpful in working with parents.

other Perspectives on the Accuracy vs. Time Dilemma Providing a high quality education to young children involves responsiveness, individualizing, reflexivity, and other qualities as previously discussed in the literature review. An 
assessment based on these practices can help lead teachers towards implementing them. However, it puts many teachers in a difficult dilemma. They are urged and guided towards practices that are very difficult to implement if there is not sufficient time, if there is little help and support, and if class sizes are large. Yet this is the reality for many, if not most, early childhood educators (Helburn, et al., 1995). Teachers' concerns about the length of the assessment must be taken seriously. Although it appears that the assessment gets less wieldy to use over time (there was a significant relationship between respondents perceptions that it takes a reasonable amount of time to administer and respondents who have used the assessment for two years or more) and although the mean and median lengths of time it takes users to administer it appears to be reasonable for this type of observational assessment, the high turn-over rate of early childhood teachers, the lack of training opportunities, and the lack of support they receive makes this an issue that needs to be addressed. Many teachers will leave their jobs or require an unreasonably long period of time before they can effectively and efficiently administer the assessment. It may be that it is unrealistic and not helpful to expect best practices, which are labor, effort, and intellectually intensive, from an underpaid, overworked, and poorly trained workforce who already have unrealistic expectations placed on them. This conflict between what we know constitutes excellence and what we can realistically expect from early childhood teachers was expressed by many respondents to the survey as well 
as by some of the experts on the curricular, item, and construct validity survey. There is no easy or quick solution to this conflict. Leaders in the field can only continue to hold out models and provide tools that reflect excellence, while advocacy efforts continue. We can also support teachers wherever they are and provide creative responses to their needs and concerns. Developing several alternative versions and formats of the assessment is one way to do this. Issues raised about the length of the assessment and the curricular and item validity of the four assessment items discussed previously (\#4, 8, 11, 12) can be addressed together. A short version of the assessment will be developed that excludes these items as well as some others that are repetitious. This will be presented as an option for users who could chose the full or short version. An example of such an assessment is presented in Appendix $G$. It contains 35 items rather than 60 and eliminates problematic and repetitious items. To respond to users who felt that the assessment needed more depth and some more advanced items, a longer version of the assessment could be developed as well. This might include adding items in the domains that contain five or fewer items, dividing certain items into two separate items, and adding a domain related to behavior.

Formatting the assessment in different ways also provides more options for teachers making it responsive to different working styles. Appendix $H$ shows the full version and the proposed short version of the assessment in a "one page" format. 
It also meets the request made by one user for a summary form that could be passed on to the next teacher.

More research is needed to further refine and develop alternative versions of the assessment (through focus groups), to determine the curricular and item validity of any new items added to the assessment as result of those focus groups or from comments from users, to explore the impact of restructuring the assessment around dispositional domains, to determine congruent validity with similar well-established assessments, and to obtain data on the reliability and utility of the new versions. Further research is also needed to determine if similar results would be found for a sample population that includes greater ethnic and geographic diversity.

\section{Conclusion}

The Oregon Assessment is a child assessment tool developed by the author in collaboration with oregon Head Start education coordinators for the purposes of measuring children's functioning, helping teachers develop individualized curriculum plans, tracking children's progress, and collaborating with parents. It was developed to meet the guidelines for developmentally appropriate assessment for young children which include naturalistic, observational, and equitable assessment practices. The constructs of interactionalism, multiple intelligences, dispositions as educational goals, and developmentally appropriate practices formed the theoretical basis for its development. By providing a measure of social 
competence, the author intended to develop an instrument that could be used in program evaluation research, particularly for Head start. As rated by carefully selected experts, The oregon Assessment was found to have an acceptable degree of curricular and item validity except for one item in the play domain. Additionally three other items, one in the self-help domain and two in the self-concept domain were found to be weaker than the others. Based on research sample used (representative of northwest U.S., not the U.S. as a whole), congruent validity between the assessment and The Mccarthy Scales for Children and The Vineland Adaptive Behavior Scales (Classroom Edition) was moderate but significant for total scores. Congruent validity using assessments more similar to The oregon Assessment might result in stronger correlations. For this same research sample, test-retest and inter-observer reliability, and four measures of internal reliability were found to be highly significant. Chronbach's alpha was .879 . The self-concept domain, traditionally considered subjective and therefore difficult to measure, was found to have the highest levels of inter-observer reliability and item reliability of any domain. More than two thirds of 114 users found the assessment to be useful and effective on a variety of dimensions including its ability to help with goal setting, curriculum planning, and tracking progress, its responsiveness to diversity, its helpfulness in working with parents, its effectiveness at measuring important indicators, its accuracy, the amount of time to administer, and more. 
The major concern raised from several of the studies was regarding the amount of time it takes to administer. Further inquiry found that respondents who used the instrument for two years or more tended to view the assessment as taking a reasonable amount of time to administer, while those who used it less tended to view it as taking too long to administer. It was also found that there is a close relationship between attitudes towards the assessment and perceptions of whether it takes a reasonable amount of time to administer. An example of a 35 item short version of the assessment was developed that would be quicker to administer and eliminates the four items of concern, as well as a number of other items. Further research was recommended to use focus groups to refine the proposed shorter version and to help develop several other versions. Users also recommended to use a variety of research strategies to determine the validity, reliability and utility of the new versions and to replicate the results for a sample population with greater ethnic and geographic diversity.

\section{outcomes and contributions}

The oregon Assessment has the potential to fill a great need for a developmentally appropriate, valid, reliable, useful, equitable, contextualized, and observational assessment tool that measures a wide range of abilities and behaviors across many developmental domains, particularly social and emotional functioning. It appears to provide an accurate, consistent, and stable measure of self-concept development. It can positively influence early childhood education programs towards more 
developmentally appropriate practices when used for its intended primary purpose as a tool to assist teachers to plan and implement individualized curriculum. Teachers now have an effective instrument to use that helps them to focus on the particular skills and behaviors of children that reflect many of their own concerns and goals. It can help them facilitate goal setting for their children, promote individualized curriculum planning, and assist them in connecting with families. If provided with a choice of several versions of the assessment that are reliable and valid, educators would have options for accomplishing these tasks in ways that more closely suit their needs and styles. Children also are potential beneficiaries as they would not be subjected to the pressure and stress resulting from high-stakes, formal assessment procedures, norm-referenced comparisons to others, nor the inappropriate educational practices that are often driven by these types of assessments. If the assessment is used effectively, children would be provided with curricula strategies that build on their strengths and are keyed to their individual needs. Greater responsiveness from teachers could only serve to benefit children.

Furthermore, The Oregon Assessment can assist Head Start and other programs with their research objectives of effectively measuring outcomes for children and determining program effectiveness for their primary goal for children: promoting social competence. 


\section{REFERENCES}

Airasian, P. (1991). Classroom assessment. New York: McGraw Hill.

Albert, R. \& Triandis, H. (1991). Intercultural education for multicultural societies: Critical issues. In L. Samovar \& R. Porter (Eds.), Intercultural communication: A reader (6th ed.). Belmont, CA: Wadsworth.

Bagnato, S. \& Neisworth J. (1981). Linking developmental assessment and curricula. Rockville, MD: Aspen Systems corp.

Barnett, D.W., Macmann, G.M., Carey, K.T. (1992, Spring). Early Intervention and the assessment of developmental skills: Challenges and directions. Topics in Early Childhood Special Education, 12(1), $21-43$

Berk, R.A. (Ed.). (1978). Criterion-referenced measurement: The state of the art. Baltimore: The Johns Hopkins University Press.

Bidell, T. (1988). Vygotsky, Piaget and the dialectic of development. Human Development, 31, 329-348.

Bowman, B.T. (1978). Sexism and racism in education. In Barbara Sprung (Ed.), Perspectives on non-sexist early childhood education. New York: Teachers College Press.

Bowman, B. T. (1992). Reaching potentials of minority children through developmentally and culturally appropriate programs. In S. Brekekamp \& $\mathrm{T}$. Rosegrant (Eds.), Reaching potentials: Appropriate curiculum and assessment for young children. (pp. 128-136). Washington, D.C.: NAEYC.

Brandt, R. (1993, April). On teaching for understanding: A conversation with Howard Gardner. Educational Leadership, $\underline{50}(7), 4-7$.

Bredekamp, S. (Ed.). (1987). Developmentally Appropriate Practice in Early Childhood Programs Serving Children from Birth through Age 8. Washington D.C.: NAEYC. 
Bredekamp, S. \& Rosegrant, T. (Eds.). (1992). Reaching potentials: Appropriate curriculum and assessment for young children. Washington, D.C.: NAEYC.

Bricker, [)., Bailey, E.J., \& Slentz, K. (1990). Reliability, validity, and utility of the evaluation and programming system for infants and young Children (EPS-1). Journal of Early Intervention, $14(2), 147-158$.

Bricker, D., \& Littman, D. (1982). Intervention and evaluation: The inseparable mix. Topics in Early Childhood Special Education, I(4), 23-33.

Bronson, M.B. (1994). The usefulness of an observational measure of young children's social and mastery behaviors in early childhood classrooms. Early Childhood Research Quarterly, 2, 19-43.

Brooks-Gunn J., \& Matthews, w.S. (1979). He \& she: How children develop their sex role identity. Englewood Cliffs, NJ: Prentice-Hall.

Cangelosi, J.S. (1990). Designing tests for evaluating student achievement. New York: Longman.

Carrasco, R.L. (1979, April). Expanded awareness of student performance: A case study in applied ethnographic monitoring in a bilingual classroom. Paper presented at Institute for Research on Teaching, East Lansing, MI. (ERIC Document Reproduction Service No. ED 250 933)

Clark, M.E. (1990). Meaningful social bonding as a universal human need. In J.W. Burton (Ed.), Conflict: Human needs theory. New York: St. Martin's Press.

Cole, J.O., \& Washington, V. (1986). A critical analysis of the assessment of the effects of Head start on minority children. Journal of Negro Education, $\underline{55}(1), 91-106$.

Comer, J. (1981). Societal change: Implications for school management. Washington, D.C.: National Institute of Education.

Cowen, E.L., Pederson, A., Babigian, H., Izzo, L.D., \& Trost, M.A. 1(1973). Long-term follow-up of early detected vulnerable children. Journal of Consulting and Clinical Psychology, 41, 438-446.

Cummins, J. (1989). A theoretical framework for bilingual special education. Exceptional Children, 50. $111-119$. 
Cunningham, J.G. (1981). Behavioral/emotional screening of Head start children. Paper presented at the Biennial Meeting of the Society for Research in child Development, Boston, MA. (ERIC Document Reproduction Service \#ED 215 751)

De Leon, J. (1990, Summer). A model for an advocacyoriented assessment process in the psychoeducational evaluation of culturally and linguistically different students. The Journal of Educational Issues of Language Minority Children, 7, 53-67.

Derman-Sparks, L. (1992). Reaching potentials through antibias, multicultural curriculum. In S. Brekekamp \& T. Rosegrant (Eds.). Reaching potentials: Appropriate curriculum and assessment for young children. (pp. 114-127). Washington, D.C.: NAEYC

Dewey, J. \& Tufts, J. H. (1932). Ethics (revised edition). New York: H. Holt \& Company.

Duckworth, E. (1987). "The having of wonderful ideas" and other essays on teaching and learning. New York: Teachers College Press.

Early Childhood Training Center (1990). The Oregon assessment for $3-5$ year olds in developmentally appropriate classrooms. Portland, OR: Author.

Early Childhood Training Center (1990). The user manual for the oregon assessment for $3-5$ year olds in developmentally appropriate classrooms. Portland, OR: Author.

Ebel, R.L. \& Erisbie, D.A. (1991). Essentials of educational measurement (Fifth edition). Englewood Cliffs, N.J.: Prentice Hall.

Eder, D., \& Hallinan, M. (1978). Sex differences in children's friendships. American Sociology Review, 43, 237-250. $=$

Esposito, A. (1979). Sex differences in children's conversations. Lanquage and Speech, 22, 213-220.

Fein, G., \& Stork, L. (1981). Sociodramatic play: Social class effects in integrated preschool classrooms. Journal of Applied Developmental Psychology, 2, $267-279$.

Elores, A., \& Riley M. (1982). The why, what, and how of a bilingual approach for young children. Lubbock, TX: Texas Tech Press. 
Forman, G.E., \& Kuschner, D.S. (1983). The child's construction of knowledge. Washington D.C.: NAEYC.

Eriedman, S. (1985). "The Mozart of psychology": "Mind in society" by L.S. Vygotsky: Implications for improving literacy. (ERIC Document Reproduction Service No. ED 277 972)

Gammage, P. (1991, March). Changing ideologies and provision in western Canada primary education. (ERIC Document Reproduction Service No. ED 331 635)

Gardner, H. (1983). Erames of mind. New York: Basic Books.

Gardner, H. (1991). The unschooled mind. New York: Basic Books.

Gifford, B. R., \& O'Connor, M.C. (1992). Changing assessments: Alternative views of aptitude, achievement, and instruction. Boston: Kluwer Academic Publishers.

Gilligan, C. (1982). In a different voice: Psychological theory and women's development. Cambridge, MA: Harvard University Press.

Ginsburg, H. (1972). The myth of the deprived child. Englewood Cliffs, NJ: Prentice-Hall.

Glanville, C,I., \& Sundberg, J. (1987). Effectiveness of teaching communication skills on acting out behaviors among four year olds in a day care center. (ERIC Document Reproduction Service No. ED 277 483)

Gleason, J.B. (1987). Sex differences in parent-child interaction. In Susan U. Philips, susan Steele, and Christine Tanz (Eds.), Language, gender, and sex in comparative perspective (pp. 189-199). Cambridge: Cambridge University Press.

Gonzalez-Mena, J. (1992). Taking a culturally sensitive approach in infant-toddler programs. Young Children, $\underline{47}(2), 8-11$.

Goodwin, M.H. \& Goodwin, C. (1987). Children's arguing. In Susan U. Philips, Susan Steele, and Christine Tanz (Eds.), Lanquage, gender, and sex in comparative perspective (pp. 189-199). Cambridge: Cambridge University Press.

Gordon, E. W. (1979). Evaluation during the early years of Head Start. In E. Zigler \& J. Valentine (Eds.), Project Head start: A legacy of the war on poverty (pp. 399-404). New York: The Eree Press. 
Grace, C. \& Stores, E.F. (1992). The portfolio and its use: Developmentally appropriate assessment of young children. Little Rock, AR: SACUS.

Griffin, A. (1994, December, 1995, January). Heart start: The emotional foundations of school readiness as challenge, lens and language for training. (pp. 5-10). In L.Eggbeer \& E. Fenichel (Eds.), Educating and supporting the infant/family work force: Models, methods and materials. 15 (3). Arlington, VA: Zero to Three/National Center for Clinical Infant Programs.

Griffing, P. (1980). The relationship between socioeconomic status and sociodramatic play among black kindergarten children. Genetic Psychology Monographs, 101, 3-34.

Gullo, D.F. (1994). Understanding assessment and evaluation in early childhood education. New York: Teachers College Press.

Hambleton, R. K. (1980, 1984). Validating the test scores. In R. A. Berk (Ed.), A guide to criterionreferenced test construction (pp. 199-230). Baltimore: The Johns Hopkins University Press.

Hale, J. (1983). Black children: Their roots, culture, and learning styles. In O. Saracho \& B. Spodek. (Eds.), Understanding the multicultural experience in early childhood education. Washington, D.C.: NAEYC.

Hazen, N., Black, B., \& Fleming-Johnson, F. (1984). Social acceptance: strategies children use and how teachers can help children learn them. Young Children, $39(6), 26-36$.

Helburn, S., Culkin, M.I., Morris, J., Moran, N., Howes, C., Phillipsen, L., Bryant, D., Clifford, R., Cryer, D., Peisner-Feinberg, E., Burchinal, M., Kagan, S.I., \& Rustici, J. (1995). Cost, quality, and child outcomes in child care centers. Denver, CO: University of Colorado at Denver.

Hicks, J.S. (1988). The Five P's replication study. (ERIC Document Reproduction Service \#ED 312 853)

Hills, T. (1987). Children in the fast lane: Implications for early childhood policy and practice. Early Childhood Research Quarterly, 2, 265-273. 
Hills, T. (1992). Reaching potentials through appropriate assessment. In S. Bradekamp \& T. Rosegrant (Eds.), Reaching potentials: Appropriate curriculum and assessment for young children (pp. 44-63). Washington, D.C.: NAEYC.

Holloway, S.D., I\& Reichhart-Erickson, M. (1988). The relationship of day care quality to children's freeplay behavior and social problem-solving skills. Early Childhood Research Quarterly, 3(1), 39-54.

Howes, C., f Earver, J. (1987). Social pretend play in 2-year-olds: Effects of age of partner. Early Childhood Research Quarterly, 2(4), 305-314.

Ivac, I. (1989). Profiles of educators: Lev S. Vygotsky. Prospects, 12(3), 427-436.

Johnson, J.E., Christie, J.E., \& Yawkey, T.D. (1987). Play and Eiarly Childhood Development. Glenview, IL: Scott, Foresman and Co.

Jones, E. (1994). Emergent Curriculum. Washington, D.C.: NAEY'C.

Kegan, R. (198:2). The evolving self: Problem and process in human development. Cambridge, MA: Harvard University Press.

Kamii, C. (1990). Achievement testing in the early gradess. Washington, D.C.: NAEYC.

Katz, L. (1990). What should young children be learning? Exchange, 76, 12-14.

Katz, L. (1991). Pedagogical issues in early childhood education. In S.L. Kagan (Ed.), The care and education of America's children: Obstacles and opportunities. Ninetieth yearbook of the National Society fir the Study of Education. (pp. 50-68). Chicago: University of Chicago Press.

Katz, L.; Chard, S. (1989). Engaging children's minds: The project approach. Norwood, N.J.: Ablex.

Kitano, M.K. (1983). Early education for Asian-American chilaren. In O. Saracho \& B. Spodek (Eds.), Understanding the multicultural experience in early childhood education. Washington, D.C.: NAEYC.

Kohlberg, I., LaCrosse, J., \& Ricks, D. (1972). The prediction of mental health from childhood behavior. In B, B. Wolman (Ed.), Manual of Psychopathology (pp. 55-81). New York: McGraw Hill. 
Ladson-Billings, G. (1992, Autumn). Reading between the lines and beyond the pages: A culturally relevant approach to literacy teaching. Theory Into Practice, 31 (4), 312-319.

Lasch, C. (1991, March/April). The lost art of political argument. Utne Reader, 44, 72.

Levy, A.K., Schaefer, L., \& Phelps, P.C. (1986). Increasing preschool effectiveness: Enhancing the language abilities of 3 - and 4-year-old children through planned sociodramatic play. Early Childhood Research Quarterly, 1(2), 133-140.

Lever, J. (1978). Sex differences in the complexity of children's play and games. Arnerican Sociological Review, 43, 471-483.

Louv, R. (1990). Childhood's future. Boston, MA: Houghton Mifflin.

Loyola, J., McBride, D., \& Loyola, L. (1991, Spring) . Cognitive and linguistic abilities of Puerto Rican bilingual children: Implications for assessment. The Journal of Educational Issues of Language Minority Children, $\underline{8}, 31-50$.

Madaus, G.F. (1988). The influence of testing on curriculum. In I.N. Tanner (Ed.), Critical issues in curriculum: 87 th vearbook of the National society for the Study of Education (pp. 83-121). Chicago: University of Chicago Press.

Maltz, D.N. \& Borker, R.A. (1982). A cultural approach to male-female miscommunication. In John J. Gumperz (Ed.), Language and social identity. Cambridge: Cambridge University Press.

Marcon, R.A. (November 1994). Doing the right thing for children: Linking research and policy reform in the District of Columbia schools. Young Children, 50(1), $8-20$.

Mayes, S.D. (1987, Winter). Assessment of preschool hyperactivity: Combining rating scale and objective observation measures. Topics in Early Childhood Special Education, $6(4), 49-61$.

McCarthy, D. (1972). Manual for the McCarthy scales of children's abilities. New York: Psychological Corporation. 
McKey, R.H. (1985, June). The impact of Head Start on children, families, and communities: Final report of the Head start evaluation, synthesis, and utilization project. Washington, D.C.: Administration for Children, Youth, and Families. (ERIC Document Reproduction Service No. ED 263 984)

Meade-Roberts, J. (1988). It's all academic. In E. Jones (Ed.), Reading, writing and talking with four, five, and six year olds (pp. 16-31). Pasadena, CA: Pacific Oaks.

Meisels, S. (1987). Uses and abuses of developmental screening and school readiness testing. Young Children, 42(4), 68-73.

Mitchell, J.V. (Ed.). (1985). The Ninth Mental Measurements Yearbook. Lincoln, NE: Boros Institute of Mental Measurements.

Monighan, P. (1985). The development of symbolic expression in preschool play and language. Paper presented at the Annual Meeting of the American Educational Research Association, Chicago, IL. (ERIC Document Reproduction Service No. ED 259 834)

Murphy, H.F., \& Wilkinson, J.E. (1980). Cognitive socialisation of 4-year-old children in nursery school. (ERIC Document Reproduction Service No. ED 201 381)

NAEYC (1988). Position statement on standardized testing of young children 3 through 8 years of age. Young Children, 43 (3), 42-47.

NAEYC (1991). Position statement: Guidelines for appropriate curriculum content and assessment in programs serving children ages 3 through 3 . Young Children. $46(3), 21-38$.

NASP (1991). Position statement: Early childhood assessment. Silver Spring, MD: National Association of School Psychologists.

Oakes, J. (1985). Keeping track: How schools structure inequality. New Haven: Yale University Press.

Paley, V.G. (1990). The boy who would be a helicopter: The uses of storytelling in the classroom. Cambridge, MA: Harvard University Press. 
Pellegrini, V.G. (1990). The relationship between kindergartners' play and achievement in prereading, language, and writing. Psychology in the schools, 17, $530-535$.

Raizen, S., \& Bobrow, S.B. (1974). Design for a national evaluation of social competence in Head Start children. Santa Monica, CA: Rand.

Ramsey, P.G. (1987). Teaching and learning in a diverse world: Multicultural education for young children. New York: Teachers College Press.।

Raven, C., \& Zigler, E. (1991). Three steps forward, two steps back: Head Start and measurement of social competence. Young Children, 年(4), 3-8.

Rigg, P., \& Allen, V. (1989). When they don't all speak English: Integrating the ESL student in the reqular classroom. Urbana, IL: National Council of Teachers of English.

Rogoff, B. (1990). Apprenticeship in thinking: Cognitive development in social context. New York: Oxford University Press.

Rosen, C.E. (1974). The effects of sociodramatic play on problem-solving behavior among culturally disadvantaged preschool children. Child Development, 45, 920-927.

Rothlein, L., Brett, A. (1987) . Children's, teachers', and parents' perceptions of play. Early Chiluhood Research Quarterly, 2(1), 45-54.

Rubin, K.H., Maioni, T.L., \& Hornung, M. (1976). Eree play behavior revisited. Child Development, 47, 414419.

Sachs, J. (1987). Young children's language use in pretend play. In Susan U. Philips, Susan Steele, and Christine Tanz (Eds.), Language, gender, and sex in comparative perspective (pp. 189-199). Cambridge: Cambridge University Press.

SACUS. (1990). Five position statements of the Southern Association on Children Under Si.r. (ERIC Document Reproduction Service No. ED 319 4931

Saifer, S. (1990). Practical solutions to practicalily every problem: The early childhood teacher's manual. St Paul, MN: Redleaf press. 
Saltz, E., Dixon, D., \& Johnson, J. ! (1977). Training disadvantaged preschoolers on viarious fantasy activities: Effects on cognitive functioning and impulse control. Child Development, 48, 367-380.

Salvia, J. \& Hughes, C. (1990), Cuirriculum-based assessment: Testing what is taught. New Yark: Macmillan Publishing Company

Saracho, O., \& Hancock, F. (1983). Mexican-American culture. In O. Saracho \& B. Spodek (Eds.1), Understanding the multicultural experience in earily childhood education (pp. 3-15). Washington, D.C.: iNAEYC.

Schaefer, E.S., \& Edgerton, M.D. (1978, August). $\underline{A}$ method and model for describing competence and adjustment: A preschool version of the Classroom Behavior Inventory. Paper presented at the Annual Meeting of the American Psychological Association, Toronto, Canada. (ERIC Document Reproduction Service No. ED 183262 )

Schutz, W. (1979). Profound simplicity. N.Y.: Bantam Books.

Schweinhart, L.J., \& Weikart, D,P. (1986). What do we know so far? A review of the Head start synthesis project. Young Children, 노( $(2), 49-54$.

Sheldon, A. (1990). Pickle fights: Gendered talk in preschool disputes. Discourse Processes, $13(1)$.

Shepard, L. (1989). Why we need better assessments. Educational Leadership, $46(7), 4-9$.

Shepard, L. (1991). The fnfluence of standardized tests on early childhood curriculum, teachers, and children. In B. Spodek \& O. Saracho (Eds.), Yearbook in early childhood education ( $p p .166-189$ ). New York: Teachers College Press. I

Shepard, "L., \& Smith, M. (1986). Synthesis of research on school readiness and kindergarten retention. Educational Leadership, 44,$78 ; 86$.

Simon, T., \& Smith, P.K. (1983). The study of play and problem solving in preschool children: Have experimenter effects been responsible for previous results?. British Journal, of Developmental Psychology, 1, 289-297.

Smilansky, s. (1968). The effects of socio-dramatic play on disadvantaged preschool children. New York: wiley. 
Smith, P.K., \& Syddall, S.) (1978). Play and non-play tutoring in preschool children. British Journal of Educational Esychology, 48, 315-325.

Smolucha, F. (1989). The'relevance of Vygotsky's theory of creative imagination for contemporary research on play. Paper presented at the National Biennial Meeting of the Society for Research in Child Development, Kansas City, MO. (ERIC Document Reproduction Service (No. ED 314 168)

Smolucha, L, \& Smolucha E . (1986, August). L.S. Vygotsky's theory of creative imagination. Paper presented at the Annual Convention of the American Psychological Association, Washington D.C. (ERIC Document Reproduction Service No. ED 274 919)

Sosa, A. (1990), Assessment of language minority students. Regional Hearing on the Education of Hispanics. San Antonio, TX: U.S. Department of Education. (ERIC Document Reproduction Service No. ED 323 799)

Sparrow, S., Balla, D., \& Cicchetti, D. (1984). Vineland adaptive behavior scales for children (classroom edition). Circle Pines, MN: American Guidance Services.

Sweeney, B. (1995, May). Personal correspondence with the author, May 15, 1995. Seattle, Washington

Tafoya, T. (1983). Coyote in the classroom: The use of American-Indian oral tradition with young children. In $O$. Saracho \& B.Spodek (Eds.), Understanding the multicultural experience in early childhood education (pp. 35-44). Washington, D.C.: NAEYC.

Thorne, B. (1986). Girls and boys together...but mostly apart: Gender arrangements in elementary schools. In Willard W. Hartup and Zick Rubin (Eds.), Relationships and development (pp. 167+184). Hillsdale, NJ: Erlbaum.

Tudge, J.: (1991, April). Peer collaboration: The case for treating the dyad as the unit of analysis. Paper presented at the Biennial Meeting of the Society for Research in Child Development, Seattle Washington. (ERIC Document Reproduction Service No. ED 336 404)

Turner, R.R. \& Bpulter, L.K. (1981). Eredicting social competence: The validity of the PIPS. Paper presented at the Annual Meeting of the American Psychological Association, Los Angeles, CA. (ERIC Document Reproduction Service \#ED 207 217) 
U.S. Department of Health and Human Services. (1984). Head start program performance standards (DHHS Publication No. OHDS 86-31131). Washington, D.C.: U.S. Government Printing Office.

Vaillant, G.E. (1977). Adaptation to life. Boston: Little, Brown and Company.

von Zuben, M.V., Crist, P.A., \& Mayberry, W. (1991). A pilot study of differences in play behavior between children of low and middle socioeconomic status. American Journal of Occupational Therapy, 45(2), 113118 .

Vygotsky, L.S. (1931). Imagination and creativity in the adolescent. Chapter 12 in the pedagogy of the adolescent from volume 4 of the collected Works of L.S. Vygotsky. Moscow: Pedagogika, 1984.

Vygotsky, L.S. (1932). Imagination and its development in childhood. Development of Higher Psuchological Eunctions. Moscow: Academy of Pedagogical Sciences, 1960 .

Vygotsky, L.S. (1978). Mind In Society. Cambridge, MA: Harvard University Press.

Vygotsky, L.S. (1981). The genesis of higher mental functions. In J.V. Wertsch $\left(\mathrm{Ed}_{\phi}\right)$, The concept of activity in Soviet psychology. Armonk, N.Y.: M.E. Sharpe.

Vygotsky, L.S. (1987). The collected works of L.S. Vygotsky volume 1: Problems of general psychology. New York: Plenum Press.

Wagner, B.J. (1988). Research currents: Does classroom drama affect the arts of language?. Language Arts, $\underline{65}(1), 46-55$.

Walton, J., \& Nuttall E. (1992). Preschool evaluation of culturally different children. In E. Nuttall, I. Romero, \& J. Kalesnik (Eds.), Assessing and screening preschoolers (pp. 223-265). Boston: Allyn and Bacon.

Wertsch, J.V. (Ed.). (1981). The concept of activity in soviet osychology. Armonk, N.Y.: M.E. Sharpe.

Wertsch, J.V. (1985). Vygotsky and the social formation of mind. Cambridge, MA: Harvard University Press. 
Wertsch, J.V. (1991). Voices of the mind: À sociocultural approach to mediated action. Cambridge, MA: Harvard University Press.

Wexler-Sherman, C., Gardner, H., \& Eeldman, D.H. (1988). A pluralistic view of early assessment: The Project Spectrum approach. Theory Into Practice, 27(1), 7783.

White, B. (1978). Experience and environment (Vol 2). Englewood Cliffs, N.J.: Prentice Hall.

Wiersma, W. \& Jurs, S.G. (1990). Educational measurement and testing (Second edition). Boston: Allyn and Bacon.

Wiggins, G. (1989). Teaching to the (authentic) test. Educational Leadership, 46(7), 41-47.

Wortham, S.C. (1990). Tests and measurement in early childhood education. Columbus, OH: Merrill Publishing Company.

Zukow, P. G. (1983). The relationship between interaction with the caregiver and the emergence of play activities during the one-word period. (ERIC Document Reproduction Services No. ED 230307 ) 
APPENDIX A

THE OREGON ASSESSMENT FOR 3-5 YEAR OLDS IN DEVELOPMENTALLY APPROPRIATE CLASSROOMS 


\section{OREGON ASSESSMENT FOR 3-5 YEAR OLDS IN DEVELOPMENTALLY APPROPRIATE CLASSROOMS}

FULL VERSION (60 ITEMS)

Version 1.5 August 1993

Early Childhood Training Center

Portland State University School of Extended Studles

P.O. Box 1491

Portland, OR 97207

(503) 725-4815 
Oregon Assessment for 3-5 Year Olds in

Developmentally Approprlate Classrooms

Name:

Program:
Bithdato:

Teachers:

\section{PLAY}

DATE \& COMMENTS/EXAMPLES

1. Engages in cooperathro (dramatic) play 12345

during Lree play/chlld cholco times

2. Actively engages in many different kinds of 122345

play activities - blocks, socto-dramatlc play.

manlpulatves, simple board games, etc.

3. is a sell-directed, molvated learner -

shows intlative in finding materials

12345

and choosing actir

4. Puts materials in proper place

12345

when finished.

5. Uses language skills to assist in

12345

directing cooperattve (dramatic) play

SELF-HELP

6. Takes care of personal grooming needs -

washes hands, brushes feeth, dresses seil

7. Wants to help her/mimsef and takes pride 12345

in dolng so

8. Cares for personal salety - uses seat belt, 12345

is not reckless on playground, in

classroom and on field tips

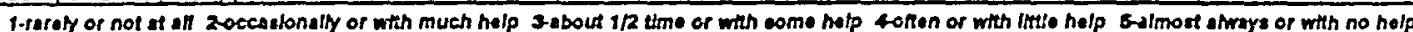

- 1990 early ChILDHOOD trunNINO center 


\section{SEELLF-C̄OONCEPT}

DATE \& COMMENTS/EXAMPLES

9. Trusts aduls who are reasonably 12345

consistent and supportive.

4.345

10. Affepts comfor from others.

$\{2345$

11. Makes eye to eye contact (provlding this 12345

(s a cuhural norm)

12. Shows approprlate caution

12345

with strangers

13. Requests help from adults and peers verbally, politely, and when actually needed

12345

14. Can take turns whth otherg (delays gratt. 12345

fication for a short period.

15. Accepts responsiblity for own

12345

actions.

12345

16. Stands up for selt assertively whon en

gaging in a conflict with others

17. Moves easily through transkions

(such as circle to snack)

12345

18. Has a realistic sense of own abilities,

strengths, weaknesses.

19. Can evaluate own actlons or beheviors

12345

12345

whithout belng too hard on her/himself

20. Raspects aduh authorty and classroom 12345 rules, but can assert own optrions,

jeas, and personality

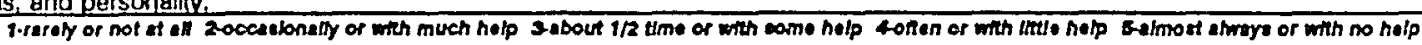

Black - 1st assessment Red-2nd assessment Green-3rd assessinent

O 1990 early childhood truanino center 
SOCIAL

1. Interacts with a variety

12345

DATE \& COMMENTS/EXAMPLES

of children

12345

22. Negotiates effectively

12345

whothers

23. Displays flexibility in roles - can be a leader and a follower and does not adriere rigldy to a sox rote

24. Verbally expresses foelings and noeds

12345

12345

25. Forms and maintains

12345

26. Displays positive relations whth aduhs, 12345

Displays postive relathons whth adults,

Including

Interdependence/independence.

are different from her/mimsell

(disabilinies, gender, skin color, eic.)

LANGUAGE/COMMUNICATION

28. Listens 10 and contributes to group di3.

cussions at meatimes \& sharing times

29. Tells experlences or simple events in a logical sequence.

30. Enjoys recting fingerplays and

singing songs.

12345

31. Communicales so s/he to

understood by others

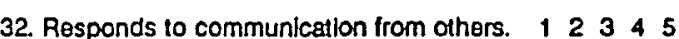

33. Intiates \& sustains conversations. 12345

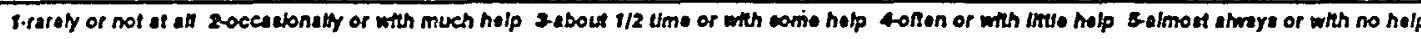

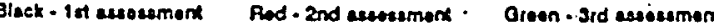

O 1900 EARLY CHILOHOOO TRNNINo CENTER 


\section{MOTOR}

34. Actively engages in and enjoys fine

moto activites (drawing, painting.

puzzles, cuting using manipulatives

35. Fregly runs, jumps, hops, climbs, rides without leguen falling or mbalance.

36. Willingly challenges own strength

and agility (fine and/or large motor)

\section{DISPOSITIONS}

Engages in and enjoys creathe acthtiles \& expression such as:

37. Creative problem solving

38. Art

2345

12345

39. Drama

12345

40. Movement

12345

41. Music 12345

- 12545

42. Displays

12345
43. Is willing to try
12345

now acthitles.

44. Enjoys taking risks whlle malntalning

12345

safety of self and others.

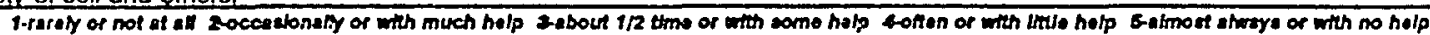

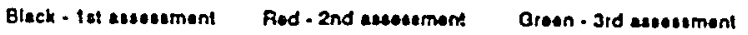

O 1090 EARLY CHILDHOOD TRUNINO CENTER 
45. Asks questions: "How, wty, where, when, 12345

whor? of gdults and children.

46. Approaches tasks with confidence

12345

47. Perseveres through a difficuth task

12345

despht setbacks such as difficut

puzzles or lego constructions.

48. Chooses acthitles during tree play and

12345

can stick with 4 for 10 minutes or more

49. Displays resourceluiness when faced with $12 \begin{array}{llll}2 & 3 & 4 & 5\end{array}$

problems or obstacles - can generate

several solutions/alternatives when

asked questions such as What else

can you do ....?"

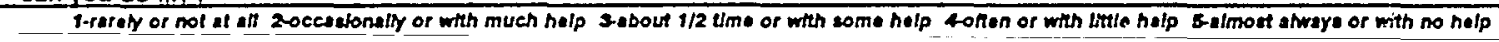

\section{COGNITIVE - MATH}

When playing with objects in the ervironment such as blocks, pegs, dishes, etc.:

50. Matches (l.e. red cars into red contalner) 12.345
51. Sorts (l.e.; red cars from
12345

blue cars)

12345

52. Seriates (i.e.; lines up care

smallest to largest)

53. Patterns (l.e.; red car, blue car,

12345

red car, blue car

54. Quantifies (i.e.; counts

cars)

2345

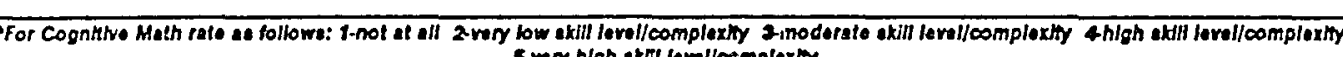

S-rory high extri terilloomploxhy

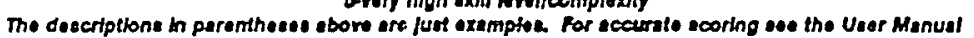

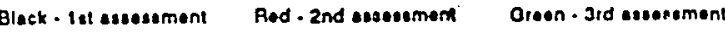

O 1990 EARLY CHILDHOOO TRUININO CENTER 
COQNITIVE - LTERACY

DATE \& COMMENTS/EXAMPLES

55. Ustens 10 entire story

during stomitme.

12345

56. Ask to be

12345

57. Shows interest in print medium enjoys looking at books, aske what signs say, atc.

58. Recognizes and names letters in environment, in print or when environment, in print or when
children's names are dictated.

59. Reads own name on drawings, chant, etc. and reads simple signs in the environment

60. Writes, draws, and/or makes things repre- 12345 semtationally, l.e.; writes name on artwork, Imltates writing during dramatic

play, draws plctures that are recog.

nizatio obtocts of paspoto.

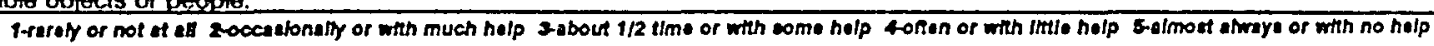

Other commente or observalions:

12345

12345

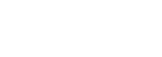

$\therefore$

$\cdot$

.

.

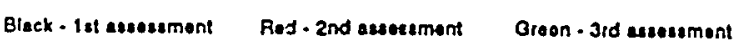

O 1950 early childhood trunino center 
- OREgON ASSESSMENT FOR 3-5 YEAR OLOS IN DEVElOPMENTAUY APPROPRLATE ClassROOMS Name: OBSERVATION FORM

Area of development: 
APPENDIX B

INDIVIDUAIIZED PLANNING FORMS 
OAEGON ASSESSMENT FOR 3.5 YEAR OLS IN DEVELOPMENTALLY APPROPRLATE CLASSROONS INDIVIDUAL CHILD PLANNING FORM

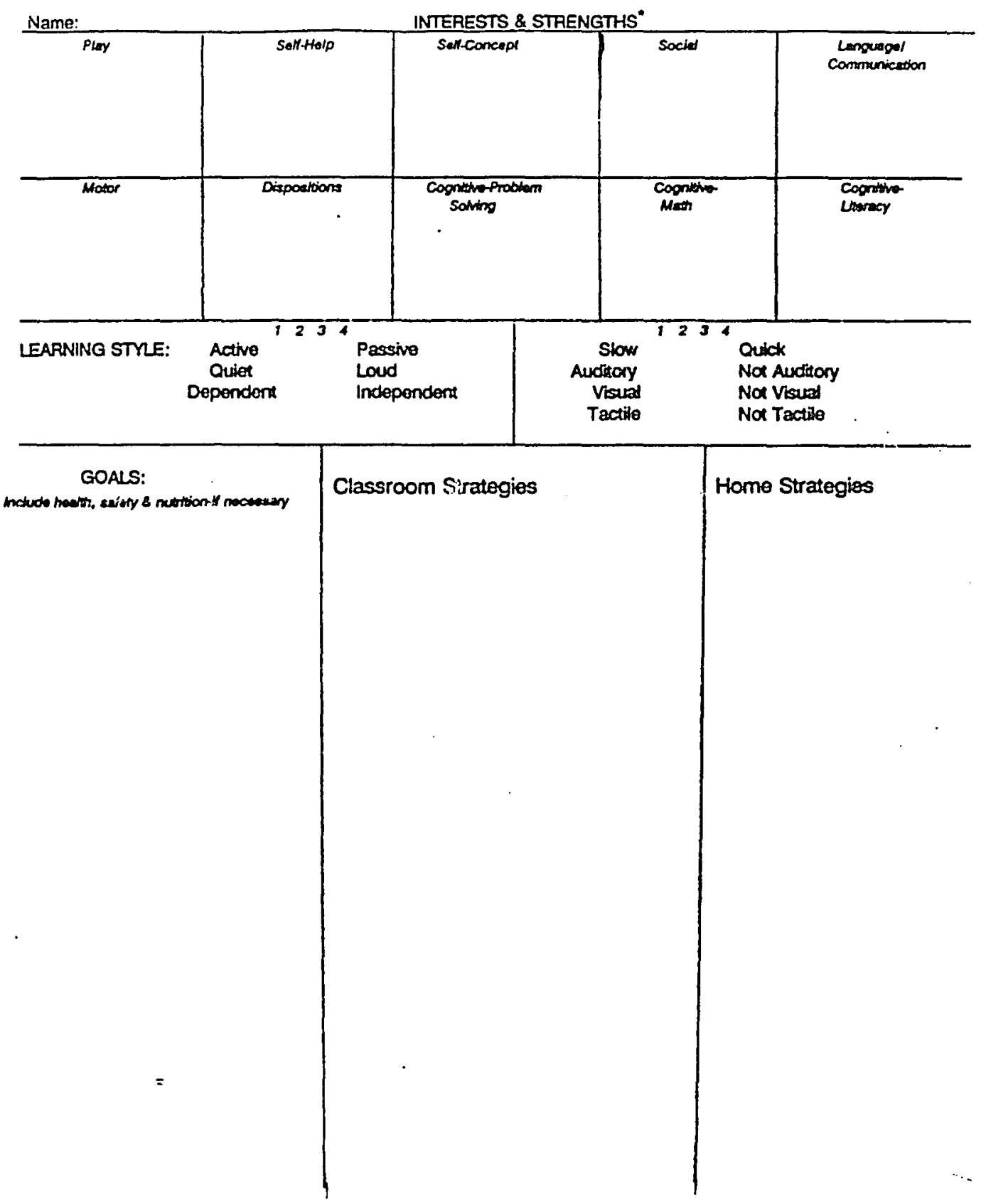


OREGON ASSESSMENT FOR 3.5 YEAR OLDS IN DEVELOPMENTALLY APPROPAIATE CLASSROOMS

CLASS PICTURE FORM

Insinctions: Use this form to transfer information from each Indlvldual Child Planning Form. Under each area of development list the children who have \& in that area and a briet description of the goals and/or strategles. You can then use the Information on this form to Individualize your daily/weekly lesson plan!

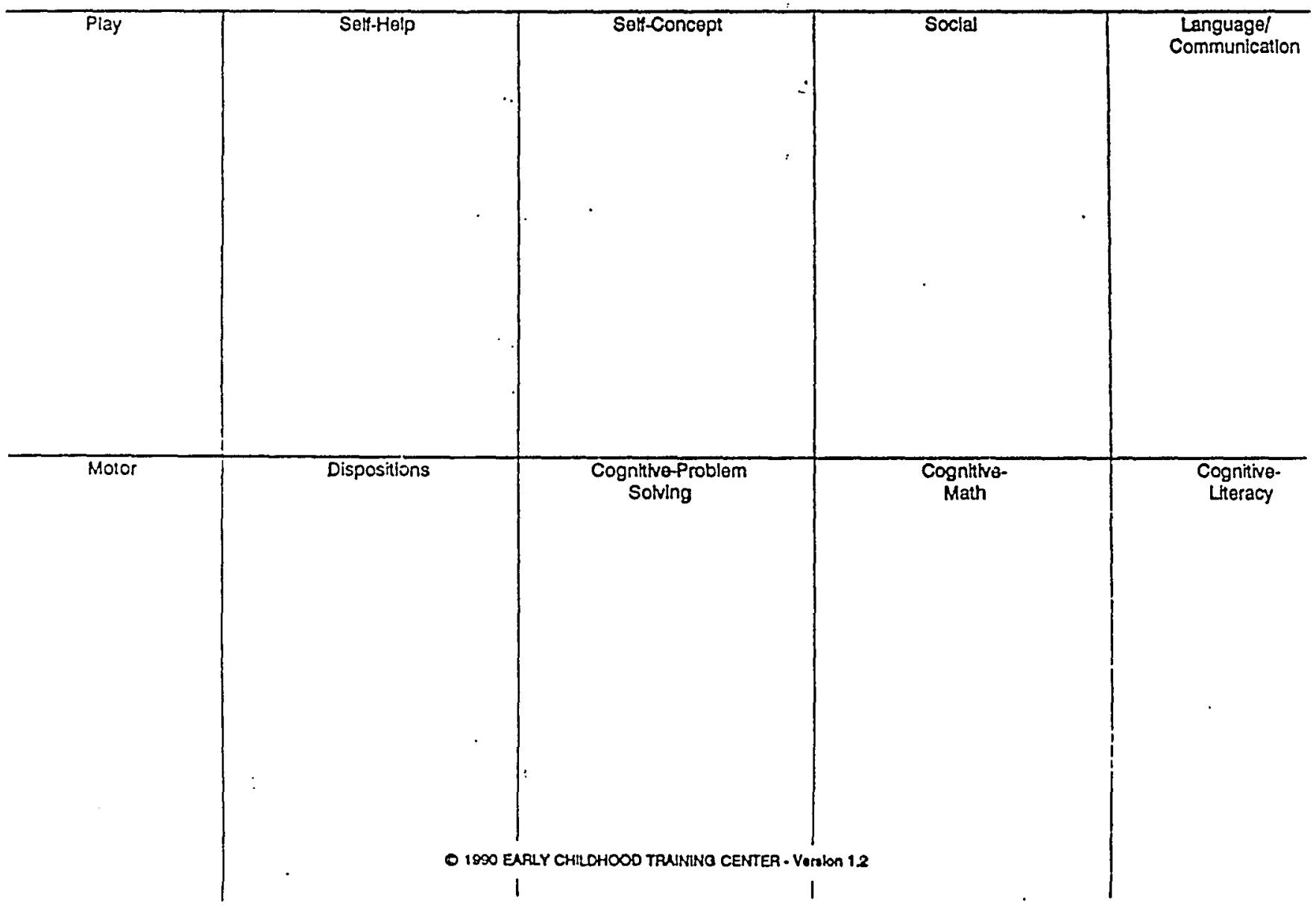




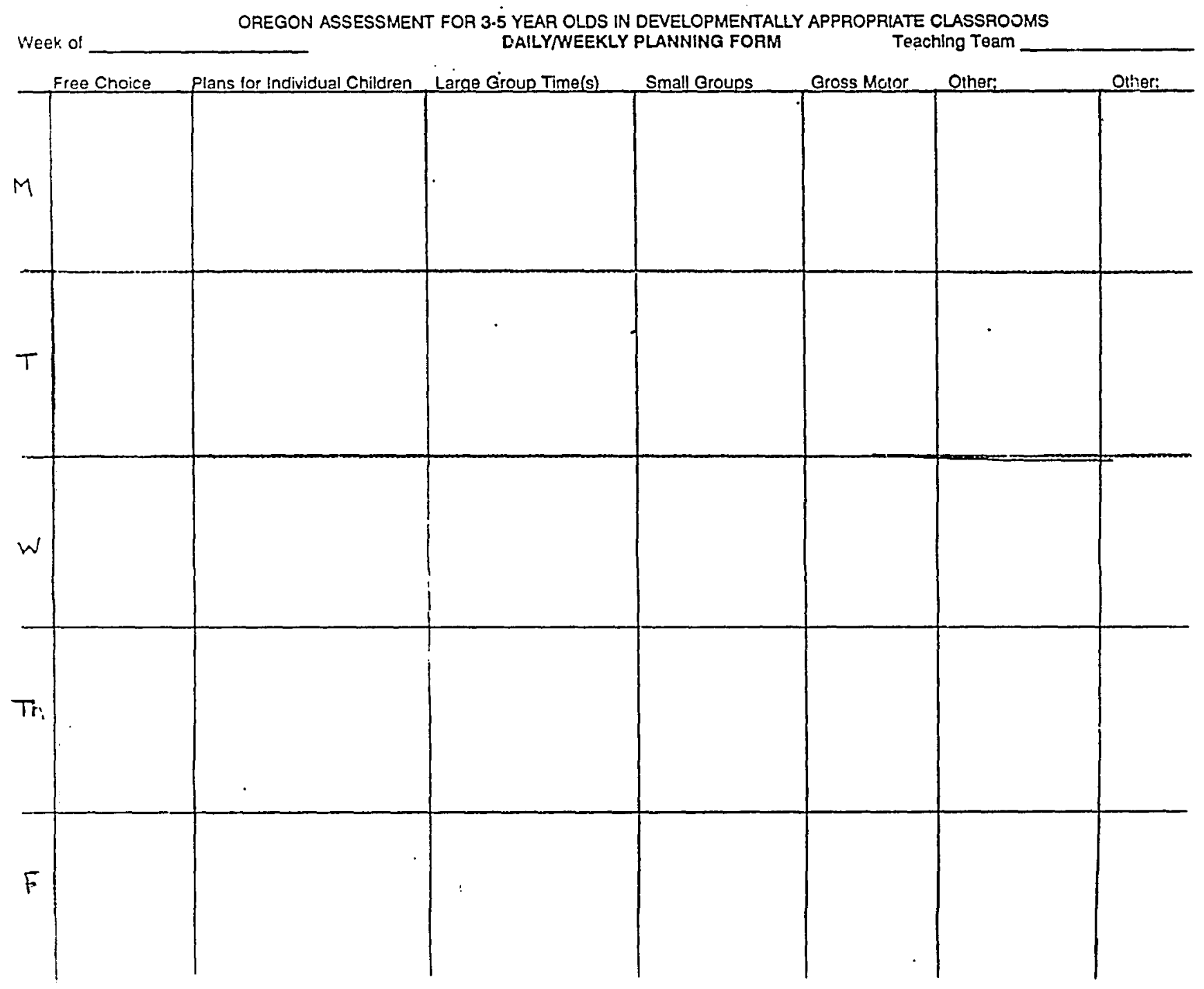

옷 
APPENDIX C

THE OREGON ASSESSMENT USER MANUAL 


\section{The Oregon Assessment for 3-5 Year Olds in Developmentally Appropriate Classrooms}

\section{USER MANUAL}

Early Childhood Training Center School of Extended Studies Portland State University

Version 1.51 


\title{
(C) 1990 Early Childhood Training Center
} All Rights Reserved

\author{
Published and printed by the Early Childhood Training Center \\ School of Extended Studies \\ Portland State University \\ P.O. Box 1491 \\ 1633 SW Park \\ Portland, OR 97202
}

(503) $752-4815$

\begin{abstract}
Use Restrictions and ordering - Any photocopying of the instrument or user manual (all or part) is strictly prohibited unless done'so by a staff member of any of the following Oregon Head Start Programs (only for use within these programs): Columbia-Pacific, Deschutes/Crook Co., Eastern Oregon, Kids \& Company, Klamath Family, Malheur Co., Mid-Columbia, Portland Public Schools ECE, Southern Oregon, Umatilla/Morrow Co., Warm Springs Tribal. All other parties must purchase The Oregan Assessment and the User Manual. Licensing agreements can be arranged for large programs or school districts. Prices and ordering information may be obtained by writing or calling the Eiarly Childhood Training Center as listed above.
\end{abstract}


Introduction $\ldots \ldots \ldots \ldots \ldots \ldots \ldots \ldots \ldots \ldots \ldots \ldots \ldots \ldots \ldots$

Backgrcund of The Oregon Assessment $\ldots \ldots \ldots \ldots \ldots \ldots \ldots \ldots \ldots$

Significance for the Early Childhood Education Profession . . . . . . . . . 2

The Importance of Assessing and Fostering Social Competence . . . . . . . . 2

An Appropriate and Effective Assessment Tool . . . . . . . . . . . . 3

An Equitable Assessment Tool . . . . . . . . . . . . . . . . 3

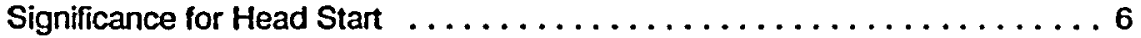

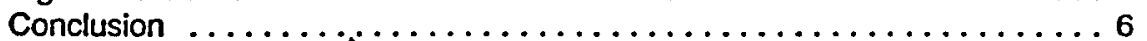

Obtaining Assessment Data . . . . . . . . . . . . . . . 7

Scoring The Oregon Assessment . . . . . . . . . . . . . . . 8

The Vital Role of Parents in Collecting Data and Scoring $\ldots \ldots \ldots \ldots$. . 8

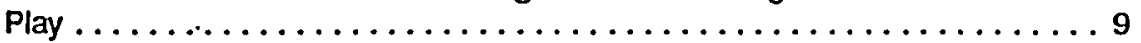

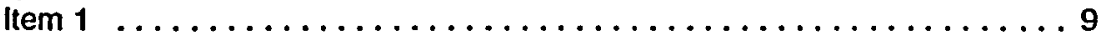

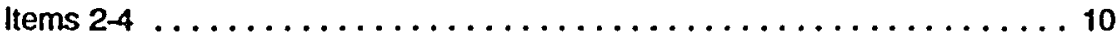

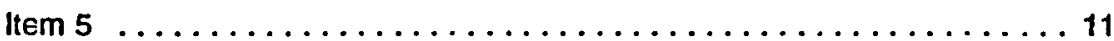

Setf-Help ............................. 11

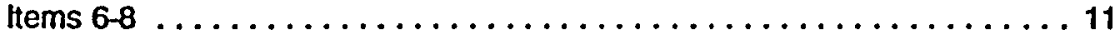

Self-Concept .............................. 12

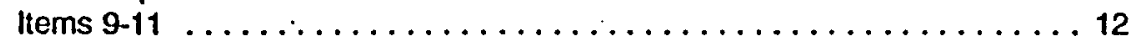

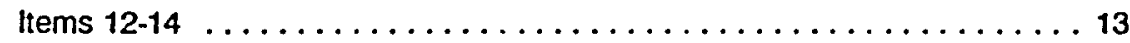

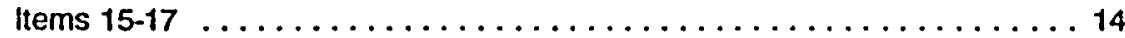

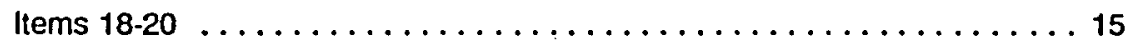

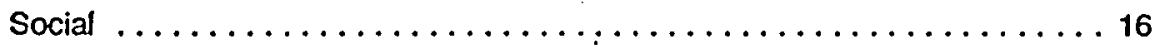

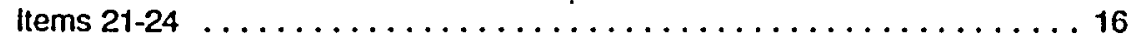

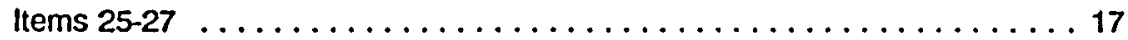

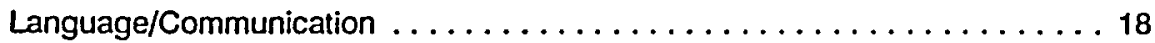

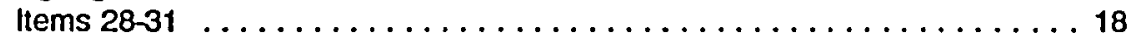

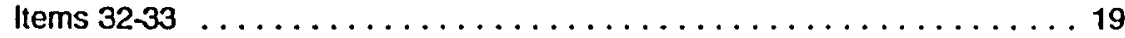

Motor ............................. 19

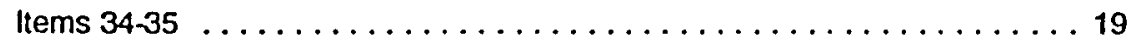

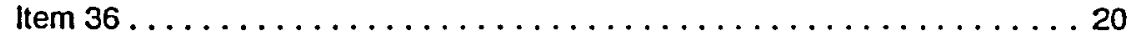

Dispositions . . . . . . . . . . . . . . . . . . . . 20

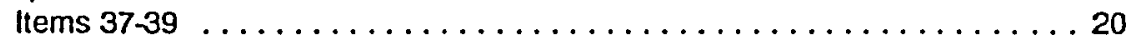

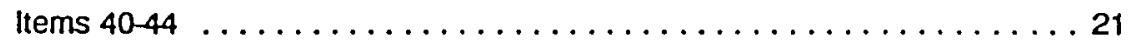

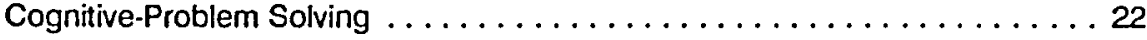

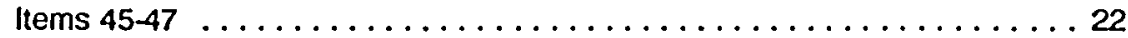

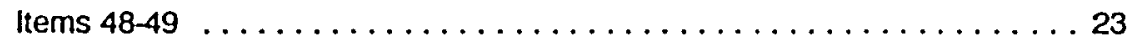

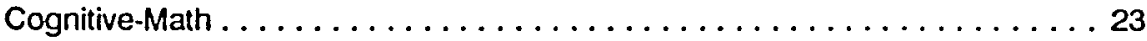

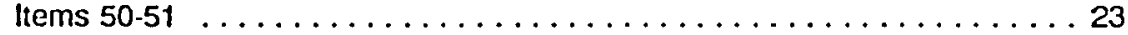

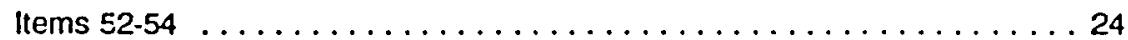

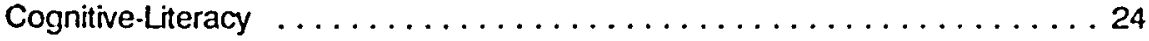

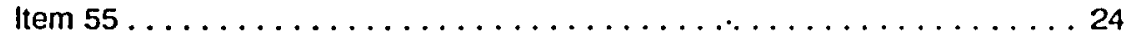

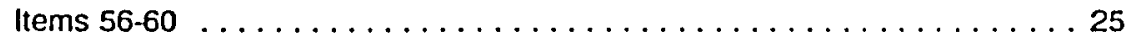

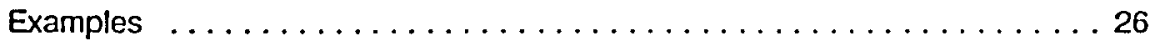

Sample Page ............................ 26a

Sample Individual Child Planning Form . . . . . . . . . . . . . 26b

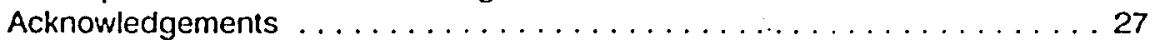

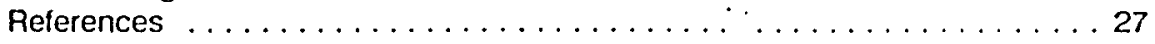




\section{Background of The Oregon Assessment}

The assessment in its current form was conceived by Steffen Saifer and developed with a team of Oregon Head Start Education Coordinators under his direction. It was field tested at three Head Start programs for one year and revised to include feedback from teachers regarding its utility, scoring, and wording of items. It is currently in its third version having been revised twiçe more in response to feedback from teachers. The impetus for its development came from a need expressed by Head Start staff for a tool for teachers to assess child progress with an emphasis on social/emotional development rather than on cognitive development. This emphasis reflects the primary goals these educators have for their children and their actual teaching practices - the development of social competence and a healthy selfconcept. Developing children's social competence is also the primary goal of the federal Head Start program (Raven \& Zigler, 1991; U.S. Department of Health and Human Services, 1984). The emphasis of the instrument is on social development, self-concept, play skills, and dispositions, balanced by cognitive skills (particularly process skills such as problem solving). The cognitive skills are oriented toward dispositions and a developmentally appropriate context (observed during play and typical classroom and home routines). All items are scored by observation in natural classroom settings, rather than in the more typical pull-out, · decontextualized mode. This method of authentic assessment reflects national early childhood education guidelines for appropriate assessment of young children (NAEYC, 1988; NAEYC, 1991).

The Oregon Assessment consists of 60 items in ten areas of development. It contains some unique features not found on most assessment tools. Among these features is an emphasis on social and intrapersonal development with less emphasis on cognitive development, an assessment of play skills, ample space to encourage descriptive comments, and a five point rating scale for each item keyed to the frequency of the observed behavior as well as the level of assistance required.

Some of these unique features stem from a theoretical construct based on the work of Lev Vygotsky (1981) who postulated that all higher mental functions develop from early social interactions. According to Vygotsky, development cannot be abstracted from a social, historicial, and cultural context. This context is reflected most clearly in the social interactions between the child and others. Many traditional assessments do not adequately examine the social functioning in children and thus fail to provide important information for educators and parents; that can be used to optimize children's growth and development. The Oregon Assessiment contains seven specifically social items and four of the six language items are related to interactive communication. In addition there two play items related to social interaction and at least 13 other items in various domains that have a clear social component. The social interaction between teacher and child, an important element in children's educational development, is assessed by a number of items on the instrument.

Vygotsky's major contribution to educational practices is his concept of the "zone of proximal development" (1978). This zone is the difference between the performance of a child left to him/herself and the performance of that same child when given assistance by an adult or more competent peer. Effective teachers focus interventions and curriculum in the zone of proximal development. The Oregon Assessment assists teachers in doing this by asking them to consider the level of assistance needed when rating children on each item.

Additional factors considered in the selection of test items were the principles of developmentally appropriate practices in early childhood education (Bredekamp, 1987; Katz, 1990) and Howard Gardner's construct of multiple intelligences (Gardner, 1983). Gardner postulated that while traditional education focuses primarily on only two types of intelligence, linguistic and logic-mathematical, equal weight should be given to five other types of intelligence - musical, spatial, bodily, intrapersonal, and interpersonal. The instrument contains items in all these areas. 
The Oregon Assessment includes a set of forms that assist teachers in setting goals for children based on their strengths and in planning an individualized curriculum. These forms are integral to the assessment in that they help teachers develop responsive and flexible curriculum practices based on the needs of the particular children they serve - a process necessary for quality education of young children (Bredekamp, 1987; Katz, 1990, Saifer, 1990; U.S. Department of Health and Human Services, 1984).

\section{Significance for the Early Childhood Education Profession}

A very important purpose of the tool is to promote socially oriented, developmentally appropriate curricula practices. An axiom in education is that "assessment drives curriculum" (Shepard, 1991). Teaching to the test is a well documented and pervasive concern in education, resulting in inappropriate curricula practices damaging to children (Hill, 1987; NAEYC 1988; Shepard, 1991; Shepard \& Smith, 1986). An assessment that has been developed from an examination of best practices and from educational goals as defiried by educators, such as The Oregon Assessment, rather than from a list of decontextualized, cognitively oriented skills, can only have a positive impact on curricula. Teachers teach to the test for good reason; they desire positive outcomes for their children and want measurements to show that they are effective educators. It is unlikely that teachers, on a large scale, can be convinced not to teach to the test. as long as test scores are used to measure teachar and program effectiveness. A more realistic approach is to change the nature of the tests in use so that "teaching to the test" will mean utilizing appropriate practices and will promote the goals for children of developing their social abilities, intrapersonal competence, positive dispositions, and cognitive processing skills.

The inevitable link between assessment and curricula can be desirable when it leads to positive outcomes (Bagnato \& Neisworth, 1981; Bricker \& Littman, 1982). The Oregon Assessment is designed to intentionally make this link for teachers through the wording of items, the nature of the items selected, and the individualized planning forms that help teachers use assessment information to write goals and then plan activities.

The Importance of Assessing and Fostering Social Competence. Utilizing The Oregon Assessment, with its emphasis on social competence, can help teachers and parents more effectively analyze interpersonal abilities and view them as critical to the healthy growth and development of their children. This, in turn, can lead to a greater emphasis on this domain in the curriculum. Fostering social competence is important for developmental, psychological, and educational reasons. Children with inadequate peer relations are at risk for mental health, social, and emotional problems later in life (Cowen, Pederson, Babigian, Izzo, \& Trost, 1973; Kohlberg, LaCrosse, \& Ricks, 1972). There is some research evidence that when teachers focus on social competence they view studerits more favorably (improving studentteacher relationships), alter instructional strategies, and increase expectations, leading to improved student performance (Carrasco, 1979). Improvements in social skills are clearly linked to gains in I.Q. (Saltz, Dixon, \& Johnson, 1977), problem solving abilities (Simon \& Smith, 1983), language development (Smith \& Syddall, 1978), and fewer acting out behaviors (Glanville \& Sundberg, 1987). Kindergarten children who engage in a great deal of sociodramatic play (the most social form of play) have higher levels of reading skills, word-writing fluency, and a variety"of language skills than children who engage in little socio-dramatic play (a better predictor than sex or socioeconomic class) (Pellegrini, 1980). The value of these findings are starting to be recognized by educators. Emphasis on the social nature of learning is one of five key components of quality primary education as identified by educators in the provinces of Alberta and British Columbia. Canada (Gammage, 1991). The Southern Association on Children Under Six identified "learning how to live comfortably with others" as one of eight needs of children that must be addressed for quality child care (SACUS, 1990). However, social competence continues to be undervalued in assessment processes which 
gives an implicit but strong message to teachers and parents that it is ultimately not very important.

The Oregon Assessment, with 27 items out of a total of 60 items clearly assessing interpersonal abilities, is an apt tool for measuring social competence. The remaining 33 items include a number with social elements such as, "Listens to entire story during storytime" (implies child is not disruptive of others) in the Cognitive-Literacy domain, and 'Displays resourcefulness when faced with problems or obstacles - can generate several solutions/alternatives when asked questions such as "What else can you do.." (which can be measured when problems or obstacles involve conflicts with other children) in the CognitiveProblem Solving domain.

An Appropriate and Effective Assessment Tool.

The assessment of young children has come under a great deal of criticism in recent years because of the potential and real negative impact that inappropriate assessment tools and misguided assessment practices can have on children (California State Department of Education, 1988; Kamii, 1990; Meade-Roberts, 1988; Meisels, 1987; NAEYC, 1988; NASBE, 1991; NASP, 1991; SACUS, 1990; Wexler-Sherman, Gardner \& Feldman, 1988). This negative impact includes children being prematurely labeled or misiabeled, misdiagnosed, retained at grade level inappropriately, misplaced in treatment programs, and/or subjected to inappropriate curriculum practices. Numerous educators and educational leadership organizations have called for the end to current assessment practices and have recommended guidelines for more effective and appropriate practices (California State Department of Education, 1988; Gammage, 1991; Kamii, 1990; Meade-Roberts, 1988; Meisels, 1987; NAEYC, 1988; NASBE, 1991; NASP, 1991; SACUS, 1990; Walton \& Nuttall, 1992; Wexler-Sherman, Gardner, \& Feldman, 1988). Common guidelines that emerge from the reports and research include: (a) the use of contextualized, observational assessment tools; (b) the assessment of a wide spectrum of abilities, skills, and behaviors across all developmental domains; (c) the use of multiple sources of information of which formal assessment is one part; (d) input from parents; (e) limiting the use of assessment results specifically to the purposes for which they are designed; ( $f$ ) the use of assessment results for individualized curriculum planning; and (g) assessment in the child's native language.

The Oregon Assessment is a tool that reflects these recommendations as a contextualized, observational tool that measures a broad array of abilities and behaviors across domains. It has been translated into Spanish. It is designed to assist teachers in developing individualized curriculum plans. Parent input is encouraged. Once reliability, validity and utility data are obtained it has the potential to be used for program evaluation and other research purposes. It is not designed for the purpose of making placement decisions.

\section{An Equitable Assessment Tool.}

Testing practices for young children have also come under a great deal of criticism for culture, race, gender, and class bias (California State Department of Education, 1988; Cummins, 1989; Ginsburg, 1972; NAEYC, 1988; NASBE, 1991; Sosa, 1990). Bias can exist in the test items themselves, in the scoring mechanism, in the procedures used to test children, or in all three. For example, many standardized tests fail to consider the level of language ability a child may have in her or his native language or dialect, or functional ability in a language that combines elements of both standard English and the child's native language/dialect. Additionally many children of color and those from low-income families, often have fewer experiences that prepare them for skills needed in test-taking. Gender differences in communication styles, moral constructs, perceptions, and cognition have been well documented, even in very young children. (Brooks-Gunn \& Matthews, 1979; Eder \& Hallinan, 1978; Esposito, 1979; Gilligan, 1982; Gleason \& Greif, 1983; Goodwin \& Goodwin, 1987; Lever, 1978; Maltz \& Borker, 1982; Sachs, 1987; Sheldon, 1990; Tannen, 1990; Thorne, 1986) and these differences may lead to 
differing interpretations of test questions and/or differing responses to test situations that will impact test scores (Bowman, 1978; Ramsey, 1987). Norms that do not reflect the population of children being tested often lead to scores that underestimate their true capabilities (California State Department of Education, 1988; Loyola, McBride, \& Loyola, 1991; Meisels, 1987).

Most studies of testing bias examine formal, standardized I.Q. or achievement tests that are administered directly (De Leon, 1990; Ginsburg, 1972; Loyola, McBride, \& Loyola, 1991) not assessments that are done through naturalistic observation. For example, direct assessment can lead to a variety of problems when used with Hispanic children (Loyola, McBride, \& Loyola, 1991). The child may lack the ability to fully understand directions or the items themselves and the child may lack verbal fluency if administered in the child's second language. Even when Spanish langladge translations are used, certain words may have different meanings in different dialects.

One particular attribute, relationality, seems to apply to children from many cultures outside of the dominant U.S. culture (Hale, 1983; Saracho \& Hancock 1983; Tafoya, 1983) as well as to females (Gilligan, 1982; Tannen, 1990). For these children, relationality (also called field-dependence, cooperation, or affiliation, depending on the researcher and the field of study) is indicative of their approach to situations, tasks, and concepts, as opposed to the more mainstream independent and anaiytic approach. An Anglo adult test-giver is likely to assume that all children have an independent cognitive style. When this test-giver interacts with a child from a co-culture whose cognitive style is more relational, the child may view the test-giver as uncaring and rejecting. This increases anxiety, already high due to the presence of a stranger or strange situation, and may lead to noncompliant behaviors or inaccurate responses. Furthermore, if the child is from a co-culture that teaches children to obey and respect adults, as many do (Kitano, 1983; Saracho \& Hancock, 1983), she or he can experience great conflict over how to please the adult test-giver while not fully understanding the task to be completed or the question to be answered. Anxiety, lack of understanding, assumptions, conflicting messages, misinterpretation of cultural-based behaviors, and other factors discussed above will lead to lower test scores in almost all cases and therefore to biased results.

When the purpose of a test is to determine placement in a program, promotion to the next grade level, or a similar "high stakes" outcome (Meisels, 1987), it is imperative that tests not reflect culture, race, gender, or class biases as misplacement can lead to severely damaging consequences that may last a life-time. (Meisels, 1987; Shepard, 1991; Shepard \& Smith, 1986). Because of the difficulty of removing bias from tests and the overrepresentation of minority groups in special education programs (Oakes, 1985) many educators are calling for the end of standardized testing for children below fourth grade (Kamii, 1990; NAEYC, 1988; Sosa, 1990).

The Oregon Assessment avoids many of the afcrementioned problems that can negatively impact equitable testing of children. Because it is an assessment of children as they are observed naturalistically, problems caused by direct, formal assessment procedures are not an issue. The teacher administers the assessment and even she/he does not ask direct questions or seek narrowly defined "correct" responses. Stranger anxiety and miscommunication due to intercultural factors are minimized. Many educators are calling for more use of this type of assessment and eliminating or reducing formal assessments as observational assessments often give more accurate, useful information (Flores \& Riley, 1982; Kamii, 1990; NASBE, 1991; SACUS, 1990). Parent information and observations of children in their own environments, observed during home visits, is included in the assessment data.

The purpose of the Oregon Assessment is for curriculum planning. Its use for "high stakes" decisions would be a misuse of the instrument. When used for research purposes, data collection would entail confidential sampling, assuring that it will not be used to judge individual children for placement purposes. Because it is a criterion-referenced instrument, norm-referenced comparisons are not possible. 
A number of items on the Oregon Assessment value the attribute of relationality and are not typically found on assessment instruments. These include 9 ('Trusts adults who are reasonably consistent and supportive"), 10 ("Accepts comfort from others"), 11 ("Makes eye to eye contact [providing this is the cultural norm']), 12 ("Is not overly friendly with strangers shows caution"), 22 ("Negotiates effectively with others"), and 26 ("Displays positive relations with adults, including a balance of dependence/interdependence/independence"). The emphasis on the social domain throughout the assessment reflects respect for children from relational co-cultures and this can lead to positive outcomes. In an ethnographic study, Carrasco (1979) documented that when an Anglo teacher focused on the social competence of an Hispanic kindergarten child (who the teacher initially viewed as low achieving) she began to see the child more favorably (improving their relationship), altered her instructional strategies, and increased her expectations, leading to improved student performance. In addition, Item 27 ('Does not show bias towards people who are different from her/himself") helps teachers focus on developing a classroom where differences are appreciated.

Language ability, language differences, and bilingualism have little negative inipact on scores for children from co-cultures, because most of the language items deal with communicative competence rather than fluency or understanding. Competence can be expressed in any language or dialect (including non-verbal languages such as American Sign Language) as observations are focused on the production of desired outcomes through language. Only when the child being assessed is unsuccessful at communicating with peer or adult who speaks a different language/dialect than the child or who cannot understand the child's attempts at speaking the language of the peer or adult is there a possibility that scores would be affected. Item 31 (see Appendix A), "Communicates so s/he is understood by others" is the most problematic in this regard. To prevent this problem, all language items should be scored while observing the child conversing with a receiver who is fluent in the same language or dlalect the child is using.

Numerous professionals in the area of intercultural education stress the important role teachers have in helping children achieve biculturalism - the ability to live successfully in their own culture and the mainstream culture reflected in schools (Albert \& Triandis, 1991; De Leon, 1990; Saracho \& Hancock, 1983). Rigg and Allen (1989) recommend using accepted constructs of development when working with children from non-dominant co-cultures as all children move through same developmental stages and they are children first and foremost with similar needs as other children. Gonzalez-Mena (1992) states that "it is never all right to go along with sexism, oppression, or abuse, even if you are told that it is cultural" $(p 8)$. For these reasons items that may conflict with certain values of some co-cultures should not be eliminated but teachers should be made aware of the possible cultural basis for the observed behaviors and show sensitivity when sharing this evaluation with parents. For example, item 23 -Displays flexibiiity in roles - can be a leader and a follower and does not adhere rigidly to a sex role", may clash with values in some families from certain Hispanic cultures. A lower score for a child from such a family may be an accurate assessment, but the comment area should include information about cultural/familial expectations for that child. Goals and strategies developed from the assessment for helping a child move beyond rigid roles also need to consider the cultural/familial expectations. Some ways to do this include assuring the child that it is OK to act differently at home than at school, both ways are good in each place; and discussing with parents that sex-role flexibility is an essential survival skill in U.S. classrooms and culture while recognizing that the opposite may be true in Hispanic culture - the goal is to support the child's effectiveness and comfort in both cultures. Specific guidelines for culturally sensitive assessment for this item, as well as other items that are "culturally loaded", are included in the scoring section of this user manual. Information related to assessing children in a way that values their cultures are written in italics in the scoring section.

The Spanish version of the assessment, when used by Spanish speaking teachers and/or Spanish speaking parents, helps create mutual respect and affiliation, factors that are 
critical to positive and effective parent/community/school relations. For families who speak Spanish with dialects other than Mexican-American, modifications may be needed. It is strongly recommended not to assess Hispanic children and children from other co-cultures until they feel comfortable in the classroom and with the teacher.

The Oregon Assessment holds great promise as a useful and effective tool to equitably assess all children. Because it is designed to be used for the specific purpose of curriculum planning through naturalistic obsenvation, it is less likely to produce biased results or harmful outcomes to children than traditional, formal assessments. The Spanish language version can assist in creating positive affiliation with Hispanic families who highly value their language.

\section{Significance for Head Start}

As described previously, The Oregon Assessment is an instrument developed in part by Head Start staff directly from a need to ineasure aspects of development and child functioning reflected in classroom practices and the goals that teachers have for children (primarily social and emotional) and reflective of best practices in early childhood education (Bredekamp, 1987). This need is further documented by Raven and Zigler (1991) who describe the historic and current failure of the Head Start administration to measure the stated central goal of Head Start, social competence. The authors call for the development of an instrument that can provide such measures. Numerous researchers and program developers have concurred that the goal of Head Start for children should continue to be the development of social competence and that Head Start research has historically failed to adequately measure this goal (Cole \& Washington, 1986; Gordon, 1979; McKey, 1985; Raizen \& Bobrow, 1974; Schweinhart \& Weikart, 1986). This assessment responds to these concerns raised from within and outside of the Head Start community.

The enthusiastic response from numerous Head Start programs for the instrument further attests to its ability to measure what Head Start staff believe is most important. The refinement of the tool and documentation of its utility, reliability and validity, will greatly increase its usefulness, particularly for research purposes in determining the efficacy of Head Start.

Although the importance of helping children develop social competence was discussed previously, this is particularly important for children from low income families, the typical population served in Head Start. Young children from families of low socioeconomic status may be at greater risk for failure due to poor social skills, as numerous studies have revealed. When these children are compared with other children, they engage in less dramatic play (Fein \& Stork, 1981; Grifting, 1980; Rosen, 1974; Smilansky, 1968; White, 1978), more parallel and solitary play (Murphy \& Wilkinson, 1980; Rubin, Maioni, \& Hornung, 1976), and more passive (non-interactive) time with adults (Murpiny \& Wiikirison, 1980).

If Head Start is to fully realize the goals of developing social competence in young children and providing evidence of its effectiveness at doing so, a reliable and valid assessment tool that focuses on the social/emotional domain will be required. The Oregon Assessment has the potential to do this.

\section{Conclusion}

The developers of The Oregon Assessment hope that it will have positive impact early childhood programs, including Head Start, and future research. It can provide a measure for child functioning specific to the theoretical construct of interactionism and the guidelines for developmentally appropriate practices. It can assess children's progress in the areas of development reflective of teachers' actual practices and goals and of the goals for children recommended by national early childhood education leadership associations and agencies.

Use of the Oregon Assessment has the potential to guide programs towards effective educational practices by positively influencing curriculum practices. The instrument contains 
implicit but strong messages regarding developmentally appropriate teaching practices by the very nature of what it measures and how it asks teachers to gather data. It has the potential to be a useful, reliable, valid, equitable, contextualized, observational assessment tool that measures a wide range of abilities and behaviors across all developmental domains, particularly social functioning.

\section{OBTAINING ASSESSMENT DATA}

Information about children to include on The Oregon Assessment comes from careful observation of children in the classroom, at home, and from information from parents. All observations are made during typical routines (i.e., eating, toileting), play situations, small group games, etc., not by directly quizzing children or asking them to perform a task in isolation. This will assure that information is authentic and assessment conditions are not stressful. Training on using The Oregon Assessment is highly recommended and can be obtained from the Early Childhuod Training Center, Portland State University - (503) 725-4815. All items should include descriptive information in the comments/examples area and/or on the Observation Forms in the back. For efficiency, all adults who work in the classroom should collect information that can later be transferred to the assessment.

The Observation Forms in back of the assessment are designed to help users collect data. One way to do this is to jot comments on Post-lt notes (the form is designed so that they will fit on it) when a useful observation is made. Put the child's name, the date, what you saw. and the item \# it relates to (if you remember it) or the area of development (motor, cognitiveliteracy, etc.) on the note. When time allows put the item number on the note and put it into one of the rows on the Observation Form organized by area of development and ordered by item number. Copy the blank form when more sheets are needed. Start a new set of Observation Forms for each new assessment period (i.e., winter, spring). With this system, the comments/examples section can be filled in before conferences from the notes or left blank as comments will be written on the notes stuck on the Observation Forms in back and will be easy to access. By leaving the comments/examples section blank information does not have to be written twice. Each teacher should develop their own procedure for collecting information that will work best for her or him. For efficiency, whenever possible collect data on several children at the same time in several domains. For example, while 4 children are . involved in dramatic play, information can be obtained about each of those children to potentially score and describe some of the items in language, play, social, self-concept, cognitive problem solving, and in some cases cognitive math (child counts the number of baby dolls to feed) or other domains.

Observations should also be made during home visits although data can only be collected on cne child at a time. Sometimes insights to behaviors (such as the cultural basis of behaviors or parents' expectations) - crucial to heiping a child in the classroom - can only be obtained at home. Include these insights when scoring items and in the comments/ example spaces, particularly when the behaviors are different at home than in the classroom. For example, eye contact (item \#11) may be encouraged with close friends and family members at home but discouraged in formal situations, with strangers and with authority figures, such as teachers. It is then reasonable to expect the child to avoid eye contact in the classroorn, particularly at first, but that eye contact will occur more often when the child feels comfortable in the classroom and the teacher evolves from an authority figure to a trusting friend. Goals and strategies for increasing eye contact would then focus on the teacher's behaviors to engender trust. Ask parents for information about items that are difficult to observe and ask for specific examples to include in the comments/examples space. Home visits provide good opportunities to do this. 
This part of the manual provides the user of the Oregon Assessment with further explanations of the items and examples of what would constitute a score of 5 and a score of 2 - except for the first item which includes examples for all scores. From this you should have no trouble determining what would constitute a score of 1,3 , or 4 for the other items. Refer to the criteria at the bottom of each page of the assessment in order to help you determine the appropriate score. The criteria (except for Cognitive-Math) are $1=$ rarely or not at all; $2=$ occasionally or with much help; $3=$ about $1 / 2$ the time or with some help; $4=0$ often or with little help; and $5=$ almost always or with no help. To help you determine scores related to help from the teacher or other adult, the first item gives examples of all possible scores based on level of assistance needed. Assistance or help refers to any physical help, verbal guidance, reminders, encouragement, or support that an adult gives for the child to engage in the activity. Physical help (i.e., phsyically guiding the child's hand to show where blocks go on the shelf) indicates a greater degree of assistance, and therefore a lower score, than verbal help (i.e., "remember to put the long, rectangle blocks on the top shelf next to the blocks that match it"), which in turn indicates a greater degree of assistance than a reminder ("put the blocks back on the shelf'). Also note that daily reminders or reminders that need to given often indicate a greater degree of assistance, and therefore a lower score, than occasional reminders.

Do not make considerations for a child's age when scoring. Just focus on the observed behaviors. There will be differences in the scores between most 3 year olds and most 5 year olds. Typical scores for many three olds will be 2's and 3's, while typical 5 year olds will have many more 4's and 5's. Some older versions of The Oregon Assessment have items that direct you to consider the age or developmental level of the child when scoring. Please ignore these directions.

Information in the scoring section on ways to assess children that values their cultures are written in italics. |

The examples used for what would constitute a score of 2 for each item are written in a form that demonstrates what a useful comment (to be written in the comments/examples space and/or on Observation Form) should look like.

Following the explanations and examples for scoring each item is an example of a completed page from the assessment and an example of an Individual Child Planning Form based on that page from the assessment.

\section{THE VITAL ROLE OF THE PARENT(S) IN COLLECTING DATA AND SCORING}

To provide full and complete information on the child being assessed, it is necessary to obtain information from parertt(s) cn the iterrs. A. good strategy, that many teachers use, is to discuss the assessment and the goal setting process (see form on page 26b) in their early interactions with parents (before or shortly after the child enters the program) as a semistructured way to obtain information about the child from the parent's observations and perspective. Clearly you would not necessarily expect parents to have complete answers for all items nor any information for some items. Information from parents that is later supported or enhanced by classroom observations provides a well-rounded set of evidence for skills or behaviors. Information from parents that appears to be contradictory to subsequent classroom observations also provides important information. For exar.iple, a parent might report that their child is assertive at home (item 16) - and you may aven observe this yourself on a home visit - but in the classroom you observe that the child is fairly passive. You would score such an item by the average between the score you would give for the "at home" behavior and the score you would give for the "in class" behavior. You would also make written notation of the two different behaviors. This provides very important information for setting goals and planning strategies to help the child be more assertive in the classroom. 
You would want to carefully consider the factors that contribute to the child being assertive in the home and determine if they can be used or developed in the classroom. Perhaps the child is the oldest one at home, but the youngest in the classroom. This tells you the child has the skills needed, but just needs some assistance in knowing that s/he can stand up for her/himself with older children. You also would want to consider the factors that may be negatively impacting the child in the classroom leading to the more passive behavior. Perhaps the child feels insecure or overwhelmed. You would then set goals that include paying more positive attention to the child and structuring the environment and the schedule more definitively. The forms on pages $26 \mathrm{a}$ and $26 \mathrm{~b}$ (examples section) show how parent input is used for goal setting and for developing strategies to remediate concerns by building on strengths and interests. Parents are the best source of information for identifying these strengths and interests.

Discussing the assessment with parents early in your relationship also provides an excellent opportunity to relate and clarify the values, philosophy and beliefs that you have about child development and educational practices. Assessments are powerful communicators of these beliefs because there is a strong message that 'what counts, gets 'counted'. In other words, if we (eciucators) believe it is important we will assess it and if we assess it we must believe it is important. Everyone who has had any formal schooling has internalized strong messages about the (usually undue) importance we place on assessment and assessment tools. But the Oregon Assessment communicates something a little different from most assessment tools about what is important. For example, the items in the cognitive literacy domain reflect more of a whole language, meaning-based approach to literacy rather than a purely skill-based approach. There is no item on this assessment that asks if children can name certain letters or sounds. The assessment as a whole reflects goals for children that emphasize social competence, self-concept development, and the development of positive dispositions (but not to exclusion of logico-mathematic cognition or certain basic skills). Through this assessment we communicate to parents that these are the things that "count".

From this framework, that reflects the principles of developmentally appropriate practice, parents should express to you what are the most important items or domains to them for their child's development. You can then make these some of the first goals to work on. When you share and discuss some possible strategies for reaching those goals, you are communicating your particular curricular approach, which is vital information for parents.

\section{EXPLANATIONS AND EXAMPLES OF EACH OF THE 60 ITEMS:}

\section{PLAY}

1. Engages in cooperative (dramatic) play during free play/child choice times.

Observe if a child engages in socio-dramatic play with at least one other child where s/he takes on a role (i.e., Mommy, bus driver, waiter) in a play scenario that is organized (i.e.; going shopping, eating at a restaurant, riding the bus to town). Cooperative play in this item refers only to this type of dramatic play situation. A child who engages in this type of play on a daily basis and does so without assistance would be given a 5 .

Example of a 1 - Lyssa does not engage in this type of play, even with teacher's verbal encouragement nor when teacher stays in dramatic play area. Prefers solitary or parallel play involving little language or interaction (puzzles, construction toys, playdough). 
Example of a 2 - Teacher usually stays with Sammy in dramatic play area and helps him firic a role to play, suggests props to use, and helps him find words to use.

$$
\text { - or - }
$$

Yolanda engages primarily in parallel play; watches others and only occasionally engages in cooperative play.

Example of a 3 - Dele typically does cooperative play two or three times a week. Will do it more often if teacher helps him find a role to enter the play.

- or -

Kyla will engage in this type of play only when the teacher is present or nearby.

Example of a 4 - Ryan does this almost everyday for short periods, prefers playing in blocks with cars \& trucks and with manipulatives.

- or -

Tasha will do this daily with an occasional suggestion from teacher that she "see what the children are doing in the dramatic play area".

2. Actively engages in many different kinds of play activities - blocks, socio-dramatic play, manlpulatives, simple board games, etc.

A child who engages in at least 5 different kinds of play such as described above (other kinds include sand or water play, pairting, and drawing) over the course of four days and without requiring assistance to do so would be given a 5 .

Example of a 2 - Simon tends to choose either dramatic play area or art area.

3. Is a self-directed, motivated learner - shows initiative in finding and choosing activities.

Examples of this include asking for materials to pursue a project or activity, easily entęring. play with other children or engaging other children in her play, and occupying herself productively with little or no direction from an adult. A child who usually does these would be given a 5 .

Example of a 2 - Carla needs a great deal of encouragement to participate in activities during free choice; this is not always successful.

\section{Puts materials in proper place when finished.}

A child who consistently puts Legos back in the container and the container back to it's appropriate place on the shelf when done using them and no one else wants to use them would be given a 5 . Sometimes the "proper place" for a toy is to leave it on the table if other children are waiting to use it or to give it directly to another child who is waiting to use it. Other examples include putting a trike back where it belongs or giving it to another child when done riding, hanging up dress-up clothes when he is done wearing them, and willingly assisting in group clean-up efforts at the end of free choice time.

Example of a 2 - Aaron usually needs to be reminded to put away items and often told where they belong before moving to a new area. 
5. Uses language skills to assist in directing cooperative (dramatic) play.

This item is evaluated during dramatic play situations as described in item \#1. Here you are looking for a child's ability to change or enrich the socio-dramatic play activity by contributing ideas. For example, while children are playing roles in a "hospital" dramatic play area, a child says "I broke my leg, I need a doctior to fix it" without prompting from an adult or more capable peer. A child who does this regularly during various dramatic play situations would be given a 5.

Example of a 2 - Jaqui often plays with others in dramatic play, but is quiet and only occasionally contributes ideas.

\section{SELF-HELP}

6. Takes care of personal grooming needs - washes hands, brushes teeth, dresses self,

A child who does this almost all the time, does it thoroughly (i.e., washes fronts and backs of hands with plenty of soap and water until clean) with no help or reminders, and cares about the basics of her/his personal appearance and cleanliness would be given a 5. Be careful to make judgements about "caring about personal appearance" based on the child's perception of appearance not yours (i.e., untied shoelaces may represent an attempt to emulate the fashions of an older sibling). Consider carefully cultural and socio-economic differences in what is considered stylish, standards of cleanliness, and access to cleaning devices or materials. Information obtained from parents during home visits will be helpful in understanding these cultural differences.

Example of a 2 - Jason requires help with putting on most clothing. Needs to be reminded most of the time to wash hands after using the bathroom.

\section{Wants to help her/himself and takes pride in doing so.}

Observe for this during self-help activities such as dressing, undressing, using the bathroom, etc. Examples of this include: Rosa struggles with buttoning a tight fitting button, she rejects an initial offer for help and then finally asks for help after trying hard for several minutes; Jaren looks carefully while her/his shoe is being tied and asks to be shown how to do it. A child who shows these behaviors regularly, and rarely asks for help when it is not really needed would be giver, a 5 . The focus for this iteni should be on the child's disposition towards helping him/herself with basic self-help skills and pride in doing for her/himself or being able to access the necessary assistance.

Example of a 2 - Marissa shows little interest in learning to zip coat or snap pants. Sometimes tries briefly and only occasionally ask for help.

8. Cares for personal safety - uses seat belt, is not reckless on playground, in classroom, and on field trips.

A child who is almost always aware of how he may physically impact others or endanger himself and adjusts behavior accordingly would be given a 5. A sense of good judgement about physical safety is being evaluated in this item. 
Example of a 2 - Leandra needs to reminded often to walk in classrooms and halls. Severa: times child strayed from the group on field trips.

\section{SELF-CONCEPT}

9. Trusts adults who are reasonably consistent and supportive.

For this item, "trust" can be shown in a number of ways including the child's willingness to stay with an adult other than her parent, to seek the teacher for comfort when distressed, or to take a risk (such as go on a field trip or climb up a high slide) with adult encouragement and support. This, of course, assumes that the adults involved have demonstrated to the child that they are trustworthy. A child who demonstrates trust consistently and on a daily basis (after a two week period of adjustment in which the adult establishes trustworthiness if the adult is new to child) would be given a 5 .

Example of a 2 - After an adult has been consistent and supportive (worthy of trust) for at least two weeks, the child is still - more often than not - reluctant to stay with the adult, does not seek him for comfort, and/or does not accept the adult's support and encouragement when taking a risk.

\section{Accepts comfort from others.}

This is similar to item \#9 but focuses specifically on the child's ability and willingness to accept comfort when it is actually needed and genuinely offered by an adult or another child. It is based on the idea that the ability to accept prosocial interactions is important as the ability to give it. Children with good self-concepts allow others in emotionally, accept their own occasional vulnerable feelings, and can rely on others to help them through difficult times. For example: Ryan hurts his leg on the playground. He then lets another child look at his cut, sympathize, help him up, and help him get an adult to clean it off. A child who does this during almost all such situations would be given a 5 .

Example of a 2 - Crystal is upset most mornings when left off by her parent. She curls up in her cubby and cries softly to herself while holding her teddy bear. After ten minutes another child comes up to her and asks if she wants to race cars together in the block area. She shouts out for the child to go away. This is a typical response to eitner children or adults who attempt to comfort her.

\section{Makes eye to eye contact (providing this is a cultural norm).}

In mainstream U.S. culture, looking directly at another while communicating is considered respectful and an indication of an adequate self-concept. Children who have a difficult time looking directly at others usually have low self-concepts, except those form cultures where not looking directly at another is a sign of respect. If the child does not make eye to eye contact due to cultural norms against this, make a note about this in the comment area. To help such a child achieve biculturalism, expect that the child would eventually do this in public situations (classrooms) but not in family situations. Inform parents about the eye to eye contact norms in U.S. culture and assure your respect of their cultural norms while helping the child understand U.S. norms. A child who in typical interactions with others looks them in the eye almost all the time would be given a 5 . 
Example of a 2 - Roberto looks down or away, rubs eyes, hides face in arms, etc. wher! interacting with others, most of the time. If the child does this only when in stressful or new situations or only with a stranger would be given a 4 and this information would be recorded in the comments/examples section.

\section{Shows approprlate caution with strangers.}

Children who are show no caution of strangers often have a strong desire to please or be liked beyond what is emotionally healthy. It can indicate an insecure sense of self much like the opposite behavior (not trusting adults who are trustworthy - item \#9). A child who typically takes a few hours or days to warm up to a substitute teacher or a visitor and acts a little shy when directly addressed by this person would be a given a 5 . Another healthy approach children can take is to ask a visitor to the classroom appropriate questions such as "What are doing here? or What's your name? And then they go about their usual activities without undue attention or concern (if the visitor does not interfere with their activities).

Example of a 2 - Teeka is not very cautious - sat on Jenny's lap immediately after meeting her and cuddled up to the substitute teacher right away. She showed some caution, however, when observer came to the classroom.

$$
\text { - or - }
$$

Julio paid a great deal of attention to observer who came to spend the day in the classroom. He tried to involve him in activities and showed him various things in the room while ignoring his usual playmates and foregoing his typical activities.

\section{Requests help from adults \& peers verbally, politely, \& when actually needed.}

For example, Michael tries to remove his finished painting from the easel. He struggles with the clamp but cannot squeeze it hard enough to remove the paper. He walks around the other side of the easel and asks the child painting there. "Will you help me take off my picture, Jonah"? A child who acts this way regularly, with few exceptions, would be given a 5 .

Example of a 2 - Suzanne typically demands help after only one failed attempt. When doing so her requests are more like orders: "Open this!" "Fix it!"

14. Can take turns with others (delays gratification for a short period).

Young children cannot be expected to wait for more than a minute or two without getting impatient or bored. However the ability to take turns with one or two others while playing a simple board game and staying engaged in the game, for example, is an emerging skill for preschoolers. A child who can do this in most situations would be given a 5. Other examples of a 5 include: Andrew often desires a particular trike to ride, but when another child is riding it he puts his name on the waiting list and plays happily in the sandbox until it is his turn.

Example of a 2 - When involved in a small group activity, Corey usually chooses not to take turns. He typically leaves the activity to play in another area of the room where there is less structure, like the water table. 
15. Accepts responsibility for own actions.

Examples of this include cleaning up spilled milk without being told to do so and without blaming another for it, helping another child fix a block structure that she accidently knocked over without being told to so by an adult, or giving up her trike when the bell on the timer rings after agreeing to do so. "Saving face" is an important value in a number of cultures. $A$ child from such a culture who is expected to "fess-up" when confronted may not do so because of this value. Help that child to follow through on actions by showing or guiding her to the appropriate behaviors, rather than blaming the child or focusing on what she did not do. A child who takes actions that indicate she accepts responsibility for her previous behavior most of the time would be given a 5 .

Example of a 2 - When asked to clean up a certain area of the room after free choice, Sarah will usually say that she did not play there, even though she did.

16. Stands up for self assertively when engaging in a conflict with others.

"Assertively" means that the child stands up for her rights in a reasonably polite, socially acceptable way that helps to solve the conflict fairly and in a way commensurate with the conflict. For example, if a another child takes a toy that she has momentarily set down, saying "Give it back to me - I wasn't done playing with it" is appropriate. However, if another child starts to grab a toy out of his hand, saying loudly "Hey, don't take it away" (while attempting to hold on to it) is an appropriately assertive response. This is opposed to "aggressively" where the child either makes clearly unreasonable demands or seeks her due in a manner that will escalate the conflict. An aggressive response to the first situation described above would be to yell at or hit the child who took the toy that was set down. Some children will be from families or cultures where confrontation is to be avoided if at all possible. Cohesion and social harmony are highly valued. If this is the case make sure it is noted in the examples/comments space and that strategies to help the child be more assertive in the classroom are done in a way that values the child's own culture. Please see pages 3-5 for more information about cultural sensitivity. A child who acts assertively, as described above, in most conflicts or potential conflicts would be a given a 5 .

Example of a 2 - Jena often says nothing when wronged. He just walks away. - or -

Misty typically hits during conflicts but is beginning to use the word "stop" with a great deal of heip from the teachers.

\section{Moves easily through transitions (such as circle to snack).}

Children with low self-concepts can have trouble during transition times when there is much movement, several directions have to be remembered and followed, there may be little direct guidance from teachers, there are many attractive distractions (toys on shelves to play with, friends to wrestle with), and self-direction is required. A child who, when moving from free choice to lunch, can pick up thoroughly, wash hands with little delay, and sit at the table (assuming these are the required steps) with few or not reminders would be given a 5 .

Example of a 2 - When Celia comes in from outside she typically throws her coat down on the floor and starts playing with puzzles or blocks unless reminded just before coming in to put 
her coat in her cubby and sit on the rug for story time.

18. Has a realistlc sense of own abilities, strengths, weaknesses.

Children with good self-concepts know their limitations as well as strengths. They will, for example, choose puzzles that are not too difficult or too hard, or choose to climb on outdoor equipment that is challenging but not so far beyond their capabilities that they will put themselves in physical danger (or will ask for an adult to be a "spotter" when using such equipment). A child who does these most of the time would be given a 5 .

Example of a 2 - Sean has no confidence in his own ability. He often asks for an teacher to help him on even simple tasks, but seems fine once he gets started.

$$
\text { - or - }
$$

No matter what the activity, Brian says he can do it and refuses help or initial directions from the teacher. Often he then requires intervention from the teacher to do it right or safely, or he has to be told directly to listen to everything before beginning.

\section{Can evaluate own actions or behaviors without being too hard on her/himself.}

Children with good self-concepts accept the limitations of their skills and appreciate what they do well or the process of improving. Such a child may write her own name and say "I make good 'B's but I'm just learning how to make the "e"; or after completing a puzzle say "That puzzle was too hard for me. It took me a long time, so next time l'll pick an easier one" (rather than blaming her own lack of ability). This goes beyond item \#18 which assesses the knowledge of strengths and weakness, by assessing the child's attitude towards her own strengths and weakness. A child who is not overly frustrated by her lack of ability and can take some pride in her abilities would be given a 5 .

Example of a 2 - Emelia tears up her paper after drawing a picture and cries, "I hate it. It doesn't look at all like a bird".

20. Respects adult authority and classroom rules, but can assert own opinions, ideas, and personality.

This item assesses if a child possesses a healthy balance between obedience and assertiveness. Mentally healthy individuals will question authority (appropriately) without necessarily defying it. See item \#16 for the definition of assertiveness. For children from cocultures where obedience to adult authority is highly valued, adjust your expectations accordingly, and note this in the examples/comments section. This item assumes that the classroom rules and directives are fair and reasonable, and that adult authority is responsive, caring, and democratic rather than arbitrary or authoritarian. Examples of this healthy balance include: a child who asks the reason for a rule or directive or requests an exception to a rule, rather than just breaking it, a child who requests that a rule be applied equitably (i.e., the teacher has to follow it too), a child who wants to create a new rule or modify an existing one. a child negotiates with the teacher rather than blindly accepting a directive. A child who can do any or all of these would be given a 5 .

Example of a 2 - lan follows rules with many reminders and before an infraction is likely to 
occur - such as being reminded to walk in the room just before going inside. He does not verbally negotiate or question the rule.

\section{SOCIAL}

\section{Interacts with a variety of children.}

A child who, over the course of a week, plays with children of both sexes (although not necessarily equally as most young children prefer to play with children of the same sex), different ethnic backgrounds, and with a variety of personalities and interests would be given a 5.

Example of a 2 - Ella spends most of her time with Leanne. They will prefer to sit together at circle and meals and be with each cther during free choice, uniess spccilicaliy separated by a teacher. Occasionally they join other girls in dramatic play activities.

\section{Negotiates effectively with others.}

Children with good social skills will negotiate fairly during a conflict or potential conflict until a satisfying solution for all involved is obtained. It requires good listening skills. For most young children negotiating is an emerging ability. An example of good negotiating skills: a child wants a turn "driving the car" in the dramatic play area. He asks for a turn, then engages in a discussion with the child using the car until they agree to set a timer for 4 minutes, sets the timer, and takes his turn when the bell rings. This child would be given a 5.

Example of a 2 - David does not take the time to listen to others when there is a conflict. With much adult help and support he is beginning listen and will stay and suggest an idea if it can happen quickly.

23. Displays flexibility in roles - can be a leader and a follower and does not adhere rigidly to a sex role.

Children who are well liked and socially skilled are flexible. They might be the "good guys" one day and "bad guys" the next day in cooperative dramatic play situations. A boy might be the Daddy one day and the Mommy the next day without feeling threatened or embarrassed. Not adhering rigidly to a sex role, includes any sex role, even if it is rot the child's own. For example, a boy who identifies himself with girls and does so rigidly and often, would score low on this item because of the stereotypical, non-flexible behaviors exhibited.

Example of a 2 - Zoe has a strong need to be the leader during cooperative play and to win and during games. She threatens not to play if she doesn't get her way. She will only play other roles if the teacher takes an active part in the activity.

24. Verbally expresses feelings and needs during interactions with others.

A requisite of good social skills and being liked by others is the ability to use words and use them expressively to get needs and desires met. A child who can say "Let's play a different gamie, that one is too scary to me" (rather than screaming to stop or runnirig from the group) or can say "I'm tired, teacher, can I just rest a little" (rather than staying behind or crying) 
would be given a 5 .

Example of a 2 - When William didn't want to go pet the goat during the field trip to the farm, he pouted and looked away from the group with his arms folded.

\section{Forms and maintains frlendships.}

The ability to make and keep a friend is an important social skill. A child who can form a particularty close connection with at least one other child, where interests are shared, the time they spent together is mutually satisfying, it is sustained over at least a month (this may have to be done by parent report for the first assessment), and they can argue and make up, would be given a 5 .

Example of a 2 - Polly does not pursue other children for friendships. Although she seems to enjoy Tina's companionship when they play together, she lets Tina make the first move almost always.

26. Displays positive relations with adults, Including a balance of dependence/interdependence/independence.

The ability to use adults as a resource, to see relationships with them as potentially mutually satisfying as well as a way to get some needs met - is what is being assessed in this item. For example, a child starts conversations with adults who he trusts, is curious about them, and expects them to be interested in him, while not depending on those aduits to meet completely meet his needs for approval, attention, and affection. This child knows how to access other resources such as other children and his inner capabilities (such as looking at a book to calm himself down). Such a child would be given a 5 .

Example of a 2 - Joachim prefers peers and tends to limit contact with adults and shy away if they make an initial contact.

27. Shows acceptance of people who are different from her/himself (disabilities, gender, skin color, etc.)

A child may show kriowledge of differences, make judgements, and ask f!ank questions about the differences and still show acceptarce. Acceptance of differences means that judgement about others, if they are made, are based on actual characteristics (friendliness or lack of, mutual interests or lack of rather than presumptions based on appearance. A child who shows acceptance by willingly playing with a suitable playmate who is different than herself would be a given a 5 .

Example of a 2 - Tara said "I don't want to play with that kid in the thing (walker)" before getting to know her, but was willing to join a group game she was in when the teacher was involved. 
LANGUAGE/COMMUNICATION

PLEASE NOTE: All language items must be assessed when the child is speaking his first language to a listener who also speaks that language (including sign language), unless the child is nearly fluent and feels very comfortable in her second language.

28. Listens to and contributes to group discussions at mealtimes \& sharing times. A good time to observe for this is during mealtimes or small group times when 4-6 children are together and there is a casual, relaxed atmosphere. This item assesses a child's ability to use language socially. For example, a teacher might ask the children about their favorite foods while eating lunch and the children enthusiastically contribute their individual opinions and respond to the opinions of others - April: "I love hot dogs, especially at the beach". Josh: "I only like hot dogs if they're barbecued and have ketchup on them, but I like any kind of pizza". A child who usually enjoys contributing to the discussion at these times and whose responses are logical and based on what others have contributed would be given a 5 .

Example of a 2 - Cinda will contribute to group discussions when she is feeling happy, but if she is upset, sad, or angry (which is more often than not) she stays silent.

\section{Tells experiences or simple events in a logical sequence.}

This item assesses a child's ability to use language to reflect the ability to keep events ordered in sequence. Children should not be quizzed directly and individually, but it should be observed when children are talking about things that are meaningful to them (they initiate the discussion) such as a recent family camping trip or a recently viewed movie. For example, a child who can describe at least 5 events of a field trip in the order in which they happened (very soon after the trip), or can retell at least 5 events from a favorite storybook in order would be given a 5 .

Example of a 2 - Zhou talked about two or three events in a row when telling about his family's trip to see fireworks. Wittr prompting from the teacher he was able to tell a few more things that happened.

\section{Enjoys reciting fingerplays and singing songs.}

This item assesses the child's disposition towards using language rhythmically and playfully. The disposition can be determined by the child's epthusiasm when participating in singing and reciting finger plays, requests for everyone to sing certain favorite ones, doing them at home, and spontaneous singing or reciting during while doing puzzles, riding trikes, or other activities. A child who usually does any or all of these would be given a 5 .

Example of a 2 - Mai listens to songs and fingerplays but does not join in. Her mother reports that she sometimes sings songs she learned at school when playing with her dolls at home.

31. Communicates so $s /$ he is understood by others.

A child's ability to get his message accross to others is assessed in this item. If the child is usually easily understood by peers and adults, even if his language is limited or his articulation is difficult to comprehend (and therefore uses facial expressions, body language. etc. to communicate), would be given a 5 . 
Example of a 2 - Manuel leaves off final consonant sounds making it difficult understand him much of the time. Has not yet developed other strategies for communicating.

- or -

Liam talks very softly and quietly. It is often difficult to hear his words and we regularly ask him to repeat himself and speak in a big voice.

\section{Responds to comminication from others.}

This item is assessing receptive language ability. A child who, almost all the time, shows that she understands what is being said to her by responding verbally or acting on a request or nodding her head, if not able to give a verbal response, would be given a 5 .

Example of a 2 - Keisha will typically turn away and not respond when you speak directly to her in small or large group situations. She is more responsive in one on one situations.

\section{Initlates \& sustains conversations.}

Like item \#28, this item assesses social language but in one on one situations. A child who regularly begins conversations with another and can keep them going for several minutes by responding to the other person's responses would be given a 5 .

Example of a 2 - Kelly will begin conversations quite often by asking other children questions such as "Where did you go?", Why did you do that?". But after they reply she'll usually walk away, attracted to something else.

\section{MOTOR}

34. Actively engages in anid enjoys fine motor activities (drawing, painting, puzzles, cutting, using manipulatives).

This item assess the disposition to engage in fine motor activities rather than skill level or ability. A child who chooses on his own at least 3 different fine motor activities over the course of 5 days and engages in some fine motor activity 4 out 5 days would be given a 5 .

Example of a 2 - Juan rarely' chooses fine motor activities (about twice a week) except for playdough \& easel painting.

35. Freely runs, jumps, hops, climbs, rides without frequent falling or imbalance.

Observe for these skills during typical ouldoor or gym activities. Do not test children on them directly or individually. Setting up a fun obstacle course can be an efficient way to observe a number of children for several of these at once. A child who can do all the above mentioned skills with little difficulty would be given a 5 .

Example of a 2 - Lydia tends to run awkwardly and climbs very cautiously. 
36. Willingly challenges own strength and agility (fine and/or large motor).

Examples of this include: A child makes many attempts to cross, hand over hand, the full length of a horizontal ladder; a child climbs to the top of an outdoor climbing structure using appropriate caution and/or help although it is difficult and a little scary; a child perseveres with a puzzle that is difficult for her; and a child strings small beads to make a necklace although stringing each bead requires condentration and attention to detail. This is done willingly, without coercion, pressure, or rewards. If a child challenges herself in fine motor but not gross motor (or vice versa) give a score $/$ of 3 and explain this in the comments/example space. A child who challenges herself, as explained, in at least two areas of fine motor (i.e., cutting and stacking blocks) and at least two areas of large motor (i.e., climbing and jumping) would be given a 5 (even if you observe each example only one time).

Example of a 2 - Although Amara does challenge herself with manipulatives, she avoids other fine motor activities and is very cautious and timid on the playground.

\section{DISPOSITIONS}

Engages In and enjoys creative jactivities \& expression such as (37-41):

For these items (37-41) the child's positive disposition towards these activities is being assessed rather than ability level. Positive dispositions can be determined by the child's requests for the activity ("When arie we going to act out a story agair?"), eagerness to participate, amount of time spent idoing the activity, and/or facial expressions during the activity (i.e., smiling, intense concentration)

\section{Creative problem solving}

Examples of this include: a child who tries many ways to balance blocks to make a tower, generates a unique and satisfactory solution to a conflict when negotiating, determines a way to climb a high playground structure, finds a way to change a dramatic play scenario to include another child, or similar problem solving skill. A child who takes pleasure in doing at least one problem solving activity in a week (does so voluntarily and spontaneously) would be given a 5.

Example of a 2 - Michael will engage in problem solving with puzzles if a teacher sits with him and talks him through it.

\section{Art}

A child who chooses at least 3 different art activities (painting at an easel, making a collage, drawing on blank paper with markers, etc.) and stays engaged in the activities for at least 5 minutes, over the course of 3 days would be given a 5 .

Example of a 2 - Lydia seldom chooses art activities, prefers blocks or dramatic play. Will occasionally paint at the easel forla minute or two.

\section{Drama}

A child who chooses to play in dramatic play scenarios either those developed by children or facilitated by a teacher (acting oult familiar stories) and actively participates in them at least 
two times in a 4 day period would be given a 5 .

Example of a 2. Noah will engage in Pizza Restaurant play if there are only a few children involved who he feels comfortable with. He does not want to be involved in other kinds of dramatic play.

\section{Movement}

When creative movement activities are offered (such as free dancing with scarves to recorded music), a child who always or almost always eagerly participates in them would be given a 5 .

Example of a 2 - Uchendu watches during group creative movement activities. He enjoys watching the others and will occasionaily free dance to music playing during free choice time if he senses that no one is watching.

\section{Music}

A child who almost always sings enthusiastically during group singing times, participates eagerly in creative rhythm sessions, and shows other signs of enjoying music (i.e., asks for certain songs, sings while working during free choice) would be given a 5 .

Example of a 2 - Indra sits quietly during group singing and usually won't sing. At times she moves her hands.

\section{Displays curiosity.}

Some ways that this can be assessed include: asks questions, likes to investigate, when introduced to something new or unique shows a strong interest by listening or watching intensely, sustains a fascination with new things, words, or ideas.

A child who does any of the above often would be given a 5 .

Example of a 2 - When a hamster was brought into the room for the first time, Marco showed little interest. He preferred to play with the familiar toys and equipment.

\section{Is willing to try new activities.}

When an activity that is new to a child is introduced, particularly if it involves something a little out of the ordinary (making footprints with bare feet in paint, holding a rabbit, making fish prints, etc.), a child who eagerly participates almost all the time would be given a 5 .

Example of a 2 - Zoe needs lots of encourage to try new things. She tried on the firefighters' clothes with a great deal verbal support and physical assistance from the teacher.

44. Enjoys taking risks while maintaining safety of self and others.

Examples of this include: climbing up the ladder of high slide while holding on firmly and asking for the teacher to wait near the bottom, jumping from a $21 / 2$ foot ledge but making 
sure the mat is positioned correctly first, and sledding down a hill but first making sure no one is below and that he is seated securely. A who almost always eagerly participates in somewhat risky activities while taking adequate safety precautions would be given a 5 .

Example of a 2 - Daniel tends to ride his trike as fast as he can with little regard for his own or others' safety. He is more cautious if reminded before hand and given several reminders while he rides.

\section{COGNITIVE - PROBLEM SOLVING}

45. Asks questions: "How, why, where, when, who?" of adults and children.

A young child should be asking many questions such as - "What does that word mean?", What does that sign say?", "When will we go on the field trip?" "How does this game work?", "Why do we brush our teeth?" "Who is that man?" "Why is his skin that color?" - as a primary method for solving problems about the nature of the world and her place in it. A child who asks questions often, asks about a variety of different topics, and shows a deep interest in the answers by listening iritently and asking follow-up questions would be given a 5 .

Example of a 2 - Latisha seldom asks questions. She mostly watches and listens.

\section{Approaches tasks with confidence - doesn't say "I can't".}

it assumed that the tasks are not very difficult or complex for the child, but present a reasonable challenge, such as a cooking project that involves following a picture recipe card, an obstacle course, or pouring juice from a pitcher. A child may not be able to do the task independently (particularly a child with a physical disability), but has the confidence in his ability to access his resources, which may include asking for assistance. He believes in his problem solving skills. Children without this confidence will often avoid challenging activities, refuse to participate, or say "I can't" before attempting it: A child who typically approaches most tasks with confidence would be given a 5 .

Example of a 2 - Max approaches tasks, especially new tasks, with caution. Will proceed if given a great deal of encouragement.

47. Perseveres through a difficult task despite setbacks such as difficult puzzles or Lego constructions.

This item assesses a child's ability to apply problem solving skills (rather than her confidence in those skills as assessed in \#46). To persevere with a challenging activity a child must use problem solving skills and access internal or outside resources. Outside resources (teachers, other children) are accessed for assistance but not to take the task over. Perseverance alone is not enough, there needs to evidence that several strategies are attempted. A child who regularly does this would be given a 5 .

Example of a 2 - After trying twice to make a ramp with blocks, Joy asked a friend to do it for her. 
48. Chooses activitles during free play and can stlck with it for 10 minutes or more.

This is an emerging ability for many 3 year olds and some 4 year olds. Important problem solving skills are demonstrated when a child can select an activity from the numerous choices available and stay with it for period of time. A child who does this on a regular basis would be given a 5.

Example of a 2 - Nick tends to wander and dabble with many different activities unless he is guided towards an activity and given assistance in getting started.

49. Displays resourcefulness when faced with problems or obstacles - can generate
several solutions/alternatives when asked questions such as "What eise can you do...?"

This item assess verbal problem solving skills in a dialogue. For example, if two children want to ride the same trike and you ask for ideas about how to solve this problem, the child can articulate at least three ideas. The ideas do not have to be "good" ideas that will all lead to a successful resolution. You are just looking for the ability to generate and anticulate them. $A$ child who can typically do this would be given a 5 .

Example of a 2 - Tamara can usually generate one solution, needs assistance to come up with more.

\section{COGNITIVE - MATH}

As indicated on the assessment, these items are scored based on skill or complexity level. Although the directions for scoring items 50-54 do not indicate to consider the amount of assistance required, do consider this when scoring as with all other items. Do not consider the child's age when scoring, as is indicated on some older versions of the assessment. The examples in parentheses on the assessment tool are only used to help explain what is meant by matching, patterning, etc., they are not to be used for scoring criteria.

When playing with objects in the environment such as blocks, pegs, dishes, etc.:

This can also be assessed when observing children put away things on the shelves, counting the number of times they ride around the track, setting the table for lunch, playing simple games, or other natural routine or play situations.

50. Matches (i.e.; led ca!s into red container;

A child who can consistently match at least three different ways (i.e., colors, shapes, pictures) and can match things that are related but not the same (baby animals to mother animals in a memory game or sounds to the corresponding pictures in a lotto game) would be given a 5 .

Example of a 2 - Alfredo can put the colored crayons in their proper containers on the shelf (six colors) but needs assistance with other types of matching. Does not grasp matching related items such as balls with bats or socks with shoes.

51. Sorts (i.e.; red cars from blue cars)

A child who can consistently sort by three attributes from a set of items with two dimensions for each attribute (i.e., blue, small cars from a set of cars and trucks that are blue or red and 
big or small) and/or sort by two attributes from a set of items with three dimensions for each attribute (i.e., green, round beads from beads that are yellow, red, or green and round, square, or rectangular) would be given a 5 .

Example of a 2 - Carmen can sort teddy bear counters color or size, but cannot sort by more than one attribute even with assistance.

\section{Seriates (i.e.; lines up cars smallest to largest)}

A child who can seriate 5 or more items in at least 3 different areas (i.e., 5 seeds by size, five rocks by weight, 5 leaves lightest to darkest) would be given a 5 .

Example of a 2 - Lance can line up small, medium and large cars. Can do 5 by size with assistance but cannot sort by weight or other area.

\section{Patterns (i.e.; red car, blue car, red car, blue car)}

A child who can pattern in sets of 4 with 3 different attributes in each set, such as: abbc, abbc or abca, abca would be given a 5 .

Example of a 2 - Brianna can follow a 2 set pattern - green bead, red bead, green bead, red bead.

\section{Quantifies (i.e.; counts cars)}

Many young children quantify using one to one correspondence. For example when a child gives crackers to a group of 3 children at the next table, he would pick up one packet and hand it to one child, pick up a second and give to the second child, etc. The ability to group is emerging. A child who can group by three (pick up three packs of crackers at once to give to the 3 children at the next table) and can count objects up to 15 would be given a 5 .

Example of a 2 - Willy can count objects to 3 before getting confused. Will count to 8 when teacher points to objects with him and counts along. Does 1 to 1 correspondence.

\section{COGNITIVE - LITERACY}

As with language items, these items should be assessed for print and stories in the child's primary language.

\section{Listens to entire story during storytime.}

The story must be age-appropriate for the child in terms of length and content. A child who does this always or almost always would be given a 5 .

Example of a 2 - Melissa will occasionally listen to a short story, after lunch. Will listen longer if sitting on an adult's lap. 
56. Asks to be read to.

This item is assessed not during a specific "book reading" time, but when a child, on his own volition, picks out a book and asks an adult to read it to him or. It may be during free choice time or during a transition, for example. Interest in the written contents of books is what is being assessed. A child who does this at least one time every 2 days would be given a 5 .

Example of a 2 - Joey only once asked to be read to, but is beginning to be interested in listening to books at storytimes. Will occasionally come over to hear a story read when an adult is reading to others in the book corner.

57. Shows Interest In print medium - enjoys looking at books, asks what signs say, etc.

Other examples include: asking for an adult to read children's names on a job chart, labels, and words on tee shirts. A child who often shows interest in any printed material would be given a 5 .

Example of a 2 - Caryn looks for her own name, but does not express interest in printed words or books.

58. Recognizes and names letters in environment, In print or when chlldren's names are dictated.

A child who can recognize his name when it is spelled verbally and can name three or more letters or letter sounds when they see it in print would be given a 5 .

Example of a 2 - Bryce cannot tell his name from other names that begin with ' $B$ ' when spelled. He recognizes and names " $B$ ", but no other letters.

59. Reads own name on drawings, charts, etc. and reads simple signs in the environment.

A child who can read her own name when placed with at least 5 other names (such as when choosing her painting fram. others on the drying rack or on a job chart) and can read at ieast two common signs when seen in their usual setting or titles of favorite books (i.e., exit, open, stop, Corduroy, Caps for Sale) would te given a 5.

Example of a 2 - Maddy can recognize her own name only with a great deal of assistance ("Your name begins with $M$, look for the $M^{*}$ ). Does not read signs.

60. Writes, draws, and/or makes things representationally, i.e.; writes name on artwork, imitates writing during dramatic play, draws pictures that are recognizable objects or people.

This item assess a child's desire to imitate common writing experiences that he sees adults do and a child's ability to represent. For many three year olds and some four year olds, the ability to represent is emerging. Representation may also be seen when the child is using clay to make an animal, or blocks to make a ship. A child who does both (imitates writing and draws or builds representationally) on a regular basis would be given a 5 . 
Example of a 2 - Chris doesn't imitate writing, occasionally represents people in his drawings.

\section{Examples}

The following are examples of a completed first page of the assessment and an Individual Child Planning Form. Three assessments for this child are included (fall, winter, and spring). The high cost of printing this in color prohibited us from doing so, but it is easier to read if it is color coded as suggested on the form.

The Individual Child Planning Form shows strengths and one goal as developed from that page of the assessment - from the fall assessment for a goal to be met before the winter assessment (about 3 months). The strategies for implementing the goal are based on the child's strengths, interests, and learning style as obtained from the assessment, observation, and parent input. More strengths would usually be included as they would be taken from the whole assessment. Ordinarily there would be several goals on this sheet for helping the child develop skills in several areas. 


\title{
Oregon Assessment for 3-5 Year Olds in Developmentally Approprlate Classrooms
}

\author{
Name: Derry Birthdate: 7-26-87 ( 4 yrs old) \\ Program: Columbia Teachers: Pat $\&$ Chris
}

PLAY

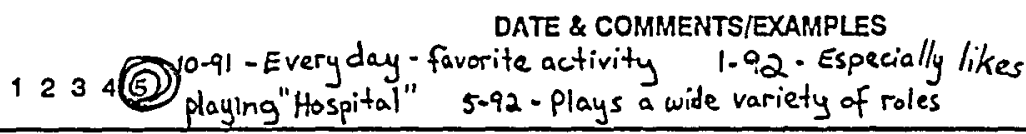

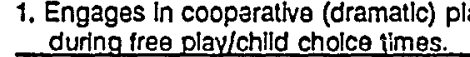

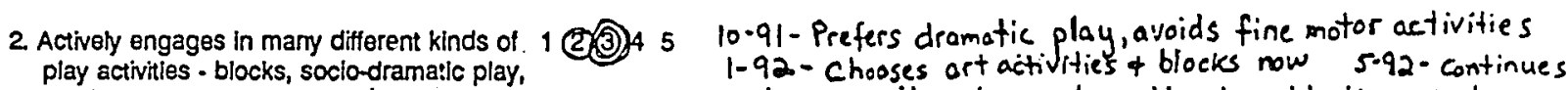
play activities - blocks, socio-dramatic play, to orimarily choose dramatic play, bloks tart

manipulatives, simple board games, etc. $10-91$ - True for activities most interested in 1-92-Broadened

3. Is a sell-directed, motivated learner- $\quad 123$ (2)(2) $\begin{aligned} & 10-91 \text { - True for activities most interested in } \\ & \text { number of activities-needs lifile or no help. }\end{aligned}$ shows initiative in finding materials $5-92$. Continues to be gelf-dirzcted in interested activities or choosing activities 123 (4) (9) $10-91$. Enjoys doing this, only needs a bit of assistance probably

4. Puts materials in proper place because still unfamiliar Wlelassrom, 1-92-Necds no assistance-

when finished $10-91$ - Follows most of the time, rarely contributes ideas. 1-92-uses

5. Uses language skills to assist in 5-92-Centributes idess often, enriches play, favorite play partner

directing cooperative (dramatic) play of many children

SELF.HELP

6. Takes care of personal grooming needs. 1234 (5)-91-Very fastidious + thorough, prefers struggling herself to Takes care of personal grooming needs. 1234 (59) $10-41$ - Very fastidious + thorough, prefers strugging herself to
asking for help. 1 -92- Likes to show others how to do it right appropriate to her/his age level.

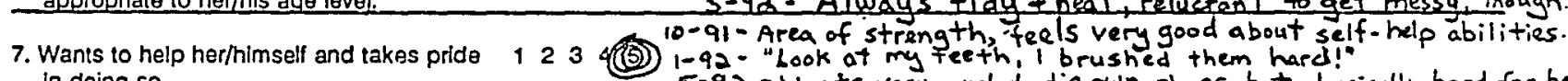
in doing so.

s-an-wants very much to tie own shoes, but physically hard for her.

8. Cares for personal safety - uses seat belt, 1 (2) (4) 10.91 - Never used seat belt before, heeds daily reminders (parents

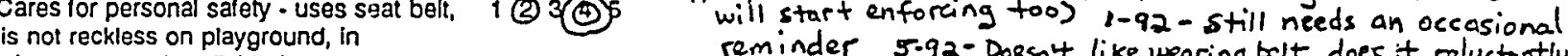
1rom, and en rield trips.

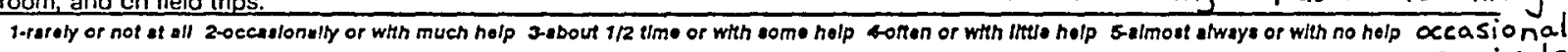

- 1990 EARLY CHILOHCOO TRANINO CENTER 


\begin{tabular}{|c|c|c|c|c|}
\hline Name: Derry & $10 / 91$ & \multicolumn{3}{|c|}{ INTERESTS \& STRENGTHS" } \\
\hline $\begin{array}{l}\text { Ploy } \\
\text { Cooperative, } \\
\text { hospital ploy }\end{array}$ & $\begin{array}{l}\text { Solltholp } \\
\text { Neat , tidy: } \\
\text { likes doing for } \\
\text { herself }\end{array}$ & Sott-Concept & Soctal & $\begin{array}{l}\text { Langungof } \\
\text { Communzcation }\end{array}$ \\
\hline $\begin{array}{l}\text { Molor } \\
\text { Parent Report: } \\
\text { Ridingtriket } \\
\text { pretanding it's a } \\
\text { motorcyde }\end{array}$ & $\begin{array}{l}\text { Oispositions } \\
\text { Parcent Report: } \\
\text { Singing "Doin } \\
\text { by the Ecey" } \\
\text {. }\end{array}$ & $\begin{array}{l}\text { Coognitiva-protilen } \\
\text { Solving }\end{array}$ & $\begin{array}{l}\text { Counnitio- } \\
\text { Mats }\end{array}$ & $\begin{array}{l}\text { Cognitim } \\
\text { Liaracy } \\
\text { Parent Report: } \\
\text { Books about whales }\end{array}$ \\
\hline ARNING STYLE: & $\begin{array}{lll}\text { Active } & x^{3} & \text { Pas } \\
\text { Quiet } x & & \text { Lou } \\
\text { Cependent } & ? & \text { Ind }\end{array}$ & $\begin{array}{l}\text { ve } \\
\text { endent }\end{array}$ & $\begin{array}{l}\text { Slow } x^{1} 23 \\
\text { Auditory } x \\
\text { visual } x \\
\text { Tactile } x\end{array}$ & $\begin{array}{l}\text { uditory } \\
\text { isual } \\
\text { actile }\end{array}$ \\
\hline
\end{tabular}

GOALS:

Include hoalth, saloty \& nutition-li nocessary

1.For item 2 - Derry will engage in more activities. such as blocks, puzzles, qart
STRATEGIES:

la. offer a variety of art activities on whale themes-collage, prints, whale-shaped paper.

b. Assist her in making a "motorcycle track" with blocks, use foy motorcycles.

c. Facilitate "whale.watching ships" made wi blocks

d. Put whale toys in water table.

e. Trade $\partial^{2}$ of my. puzzles for moturcycle puzzle + dolphin puzzle from ether classrooms

F. Sing "Down by the Bing" \& other songs and play tapes while Desry + adult play together w blocks, puzzles, ctc.

As obtained from parent input and from the assessement

O 1990 EARLY CHALOHOOO TRUNING CENTER - Verzion 12

1 
Acknowledgements

Special thanks to Angie Henning, Jan Elyse Witt, and the staff of Kids \& Company Head Start, Lebanon, Oregon for collecting examples of assessment items, believing in the value of the assessment, and using it so well for the benefit of children and families. Also thanks to Debra Blankenship for her support, enthusiasm, insights, and valuable feedt)ack.

\section{References}

Albert, R. \& Triandis, H. (1991) Intercultural education for multicultural societies: Critical issues. In L. Samovar \& R. Porter (Eds.), Intercultural communication: A reader (6th ed.). Belmont, CA: Wadsworth.

Bagnato, S. \& Neisworth J. (1981). Linking developmental assessment and curricula. Rockville, MD: Aspen Systems Corp.

Bowman, B.T. (1978). Sexism and racism in education. In Barbara Sprung (Ed.), Perspectives on non-sexist early childhood education. New York: Teachers College Press.

Bredekamp, S., ed. (1987). Developmentally Appropriate Practice in Earty Childhood Programs Serving Children from Birth through Age 8, Washington D.C.: NAEYC.

Bricker, D., Bailey, E.J., \& Slentz, K. (1990). Reliability, Validity, and Utility of the Evaluation and Programming System: For Infants and Young Children (EPS-1). Journal of Early Intervention, 14(2), 147-158.

Bricker, D. \& Littman, D. (1982). Intervention and evaluation: The inseparable mix. Topics in Earty Childhood Special Education, 1(4), 23-33.

Brooks-Gunn J. \& Matthews, W.S. (1979). He \& she: How children develop their sex role identity. Englewood Cliffs, NJ: Prentice-Hall.

Cangelosi, J.S. (1990). Designing tests for evaluating student achievement. New York: Longman.

Carrasco, R.L. (1979. April). Expanded awareness of student performance: A case study in applied ethnographic monitoring in a bilingual classroom. Paper presented at Institute for Research on Teaching, East Lansing, MI. (ERIC Document Reproduction Servicel No. ED 250 933).

Cole, J.O. \& Washington, V., (1986). A critical analysis of the assessment of the effects of Head Start on minority children. Journal of Negro Education, 55(1), 91-106.

Cowen, E.L, Pederson, A., Babigian, H., Izzo, L.D., Trost, M.A. (1973). Long-term follow-up of early detected vulnerable children. Journal of Consulting and Clinical Psychology, 41, $438-446$.

Cummins, J. (1989). A theoretical framework for bilingual special education. Exceptional Children, 56, 111-119.

De Leon, J. (1990, Summer). A modal for an advocacy-oriented assessment process in the psychoeducational evaluation of culturally and linguistically different students. The Journal of Educational Issues of Language Minority Children, ㄱ. 53-67.

Early Childhood Training Center. (1990). The Oregon assessment for $3-5$ year olds in developmentally appropriate classrooms. Portland, OR: Author.

Eder, D. \& Hallinan, M. (1978). Sex differences in children's friendships. American Sociology Review, 43, 237-250.

Esposito, A. (1979). Sex differences in children's conversations. Lanquage and Speech. 22 , 213-220.

Fein, G. \& Stork, L. (1981). Sociodramatic play: Social class effects in integrated preschool classrooms. Journal of Applied Developmental Psychology, 2, 267-279.

Flores, A. \& Riley M. (1982). The why. what, and how of a bilingual approach for young children. Lubbock, TX: Texas Tech Press.

Gammage, P. (1991, March). Changing ideologies and provision in western Canada primary education. (ERIC Document Reproduction Service No. ED 331 635) 
Gardner, H. (1983). Frames of mind. New York: Basic Books.

Gilligan, C. (1982). In a different voice: Psychological theory and women's developmerit. Cambridge, MA: Harvard University Press.

Ginsburg, H. (1972). They myth of the deprived child. Englewood Cliffs, NJ: Prentice-Hall.

Glanville, C.L \& Sundberg, J. (1987). Effectiveness of teaching communication skills on acting out behaviors among four year olds in a day care center. (ERIC Document Reproduction Service No. ED 277 483)

Gleason, J.B. (1987). Sex differences in parent-child interaction. In Susan U. Philips, Susan Steele, and Christine Tanz (Eds.), Language, gender, and sex in comparative perspective (pp.189-199). Cambridge: Cambridge University Press.

Gonzalez-Mena, J. (1992). Taking a culturally sensitive approach in infant-toddler programs. Young Children, 47(2), 8-11.

Goodwin, M.H. \& Goodwin, C. (1987). Children's arguing. In Susan U. Philips, Susan Steele, and Christine Tanz (Eds.), Language, gender, and sex in comparative perspective (pp.189-199). Cambridge: Cambridge University Press.

Gordon, E. W. (1979). Evaluation during the early years of Head Start. In E. Zigler \& d. Valentine (Eds.), Project Head Start: A legacy of the war on poverty. (pp.399-404). New York: The Free Press.

Griffing, P. (1980). The relationship between socioeconomic status and sociodramatic play among black kindergarten children. Genetic Psychology Monographs, 101, 3-34.

Hale, J. (1983). Black children: Their roots, culture, and learning styles. In O. Saracho \& B. Spodek (Eds.), Understanding the multicultural experience in early childhood education. Washington, D.C.: NAEYC.

Hill, T. (1987). Children in the fast lane: Implications for early childhood policy and practice. Early Childhood Research Quarterly, 2, 265-273.

Kamii, C. (1990). Achievement testing in the early grades. Washington, D.C.: NAEYC.

Katz, L. (1990). What should young children be learning?. Exchange, 76, 12-14.

Kitano, M.K. (1983). Early education for Asian-American children. In O. Saracho \& B. Spodek (Eds.), Understanding the multicultural experience in early childhood education. Washington, D.C.: NAEYC.

Kohlberg, L, LaCrosse, J., Ricks, D.(1972). The prediction of mental health from childhood behavior. In B.B. Wolman (Ed.) Manual of Psychopathology. New York: McGraw Hill.

Lever, J. (1978). Sex differences in the complexity of children's play and games. American Sociological Review, 43, 471-483.

Loyola, J., McBride, D., \& Loyola, L (1991, Spring). Cognitive and linguistic abilities of Puerto Rican bilingual children: Implications for assessment. The Journal of Educational Issues of Language Minority Children, $\underline{8}, 31-50$.

Maltz, D.N. \& Borker, R.A. (1982). A cultural approach to male-female miscommunication. in John J. Gumperz (Ed.), Language and social identity. Cambridge: Cambridge University Press.

McKey, R.H. (1985, June). The impact of Head Start on children, families, and communities: Final report of the Head Start evaluation, synthesis, and utilization project. Washington, D.C.: Administration for Children, Youṭh, and Families. (ERIC Document Reproduction Service No. ED 263 984)

Meade-Roberts, J. (1988). It's all academic. In E. Jones (Ed.). Reading, writing and talking with four, five, and six year olds. Pasadena, CA: Pacific Oaks.

Meisels, S. (1987). Uses and abuses of developmental screening and school readiness testing, Young Children, 42(4), 68.73.

Mitchell, J.V. (Ed.). (1985). The Ninth Mental Measurements Yearbook. Lincoln, NB: Buros Institute of Mental Measurements. 
Murphy, H.F. \& Wilkinson, J.E. (1980). Cognitive socialisation of 4-year-old children in nursery school. (ERIC Document Reproduction Service No. ED 201 381)

NAEYC. (1988). Position statement on standardized testing of young children 3 through 8 years of age. Young Children, 43(3),42-47.

NAEYC. (1991). Position statement: Guidelines for appropriate curriculum content and assessmemt in programs' serving children ages 3 through 8 . Young Children. $46(3)$, 21-38.

NASP. (1991). Position statement: Early childhood assessment. Silver Spring, MD: Author.

Oakes, J. (1985). Keeping track: How schools structure inequality. New Haven: Yale University Press.

Pellegrini, V.G. (1990). The relationship between kindergartners' play and achievement in prereading, language, and writing. Psychology in the Schools, 17, 530-535.

Raizen, S. \& Bobrow, S.B. (1974), Design for a national evaluation of social competence in Head Start children. Santa Monica, CA: Rand.

Ramsey, P.G. (1987). Teaching and learning in a diverse world: Multicultural education for young children. New York: Teachers College Press.

Raven, C. \& Zig!er, E. (1991). Three steps forward, two steps back: Head Start and the measurement of social competence. Young Children, 46(4), 3-8.

Rigg, P. \& Allen, V. (1989) When they don't all speak english: Integrating the ESL student in the regular classroom. Urbana, IL: National Council of Teachers of English.

Rosen, C.E. (1974). The effects of sociodramatic play on problem-solving behavior among culturally disadvantaged preschool children. Child Development, 45, 920-927.

Rubin, K.H., Maioni, T.L. \& Hornung, M. (1976). Free play behavior revisited. Child Development, 47, 414-419.

Sachs, J. (1987). Young children's language use in pretend play. In Susan U. Philips, Susan Steele, andl Christine Tanz (Eds.), Language, gender, and sex in comparative perspective (pp.189-199). Cambridge: Cambridge University Press.

SACUS. (1990). Five position statements of the Southern Association on Children Under Six. (ERIC Document Reproduction Service No. ED 319 493).

Saifer, S. (1990). Practical solutions to practically every problem: The early childhood teacher's mariual. St Paul, MN: Redleaf Press.

Saltz, E., Dixon, D. \& Johnson, J. (1977). Training disadvantaged preschoolers on various fantasy activities: Effects on cognitive functioning and impulse control, Child Development, 48, 367-380.

Saracho, O. \& Hanicock, F. (1983). Mexican-American culture. In O. Saracho \& B. Spodek (Eds.), Understanding the multicultural experierice in early childhood education. Washington, D.C.: NAEYC.

Schweinhart, L.J. \& Weikart, D.P. (1986). What do we know so far? A review of the Head Start synthesis project. Young Children, 41(2), 49-54.

Sheldon, A. (1990). Pickle fights: Gendered talk in preschool disputes. Discourse Processes, $13(1)$.

Shepard, L. (1991). The influence of standardized tests on early childhood curriculum, teachers, and children. In B. Spodek \& O. Saracho (Eds.), Yearbook in early childhood education (pp. 166-189). New York: Teachers College Press.

Shepard, L., \& Smith, M. (1986), Synthesis of research on school readiness and kindergarten retention, Educational Leadership, 44, 78-86.

Simon, T. \& Smith "P.K. (1983). The study of play and problem solving in preschool children: Have experimenter effeqts been responsible for previous results?. British Journal of Developmental Psychology, 1, 289-297. 
Smilansky, S. (1968). The effects of socio-dramatic play on disadvantaged preschool children. New York: Wiley.

Smith, P.K. \& Syddall, S. (1978). Play and non-play tutoring in preschool children. British Journal of Educational Psychology, 48, 315-325.

Sosa, A. (1990). Assessment of language minority students. Regional Hearing on the Education of Hispanics. San Antonio, TX: U.S. Department of Education. (ERIC Document Reproduction Service No. ED 323 799)

Tafoya, T. (1983). Coyote in the classroom: The use of American-Indian oral tradition with young children. In O. Saracho \& B. Spodek (Eds.), Understanding the multicultural experience in early childhood education. Washington, D.C.: NAEYC.

Thorne, B. (1986). Girls and boys together...but mostly apart: Gender arrangements in elementary schools. In Willard W. Hartup and Zick Rubin (Eds.), Relationships and development (pp. 167-184). Hillsdale, NJ: Erlbaum.

U.S. Department or Health arid Human Services. (1984). Head Start program performance standards (DHHS Publication No. OHDS 86-31131). Washington, D.C.: U.S. Government Printing Office.

Vygotsky, LS. (1978). Mind in society. Cambridge, MA: Harvard University Press.

Vygotsky, L.S. (i981). The genesis of higher mental functions. In J.V. Wertsch (Ed.). The concept of activity in Soviet psychology. Armonk, N.Y.: M.E. Sharpe.

Walton, J. \& Nuttall E. (1992). Preschool evaluation of culturally different children. In E. Nuttall, I. Romero, \& J. Kalesnik, Assessing and screening preschoolers. Boston: Allyn and Bacon.

Wexler-Sherman, C., Gardner, H., \& Feldman, D.H. (1988). A pluralistic view of early assessment: The Project Spectrum approach. Theory Into Practice, 27(1), 77-83.

White, B. (1978). Experience and Environment (Nol 2). Englewood Cliffs, N.J.: Prentice Hall. 
APPENDIX D

RUSSIAN AND SPANISH VERSIONS 


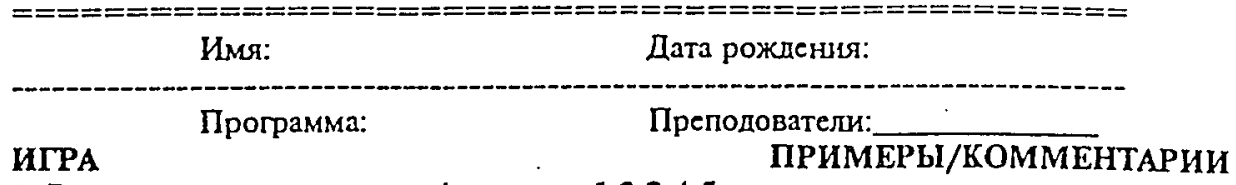

1. Вовлечение в совместную /драмати- 12345

ческуго/ игру во время свобопного вы6ора ребенка.

2. Активнос вовлеченис в итры разичныгх випов и типов /сопиально-драматические, 12345 сбыкновенињіс мағппулятивные итры и т. л.

3. Проявляет ли целеустремтеннац учапийся инипиативу в поиске материалов и видов деятельности 12345

\section{CAMOОБС ЛУУЖИВАНИЕ}

4. Заботится сам о себе, о своем внешнем виде, чистит зубы, моет руки, одевается сам

5. Желает заботиться о себе и горштся этим 12345

\section{CAMOCO3НAHИE}

6. Доверяет взроспьм, которые последоватељьы

и разуманы в свосй поднержке

7. Просит помоши у взроспьх, когда действитељьн в ней нуждается, 12345 интересуется в словах, в вежшивой форме

8. Признаст и несет ответственность 3 а свои поступи

\section{5}

9. Быстро и легко перехошт через изменендя деятельности /натример от крута к приему пиши/

10. Уважает авторитет учителя и правита поведення в ктассе, но в то же время может изложить свою точку зрсния, свои мысли по какохгу-либо повопу.

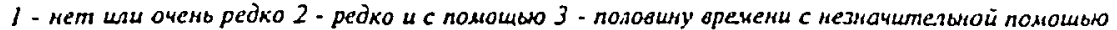

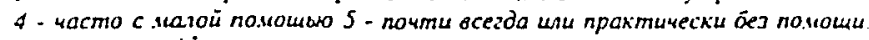


COЩНАЛЬНЫЕ

11. Активно общастся с окрухалошпоми

12345

12. Прояваляет пибкость в исполнени ролей, легко может быть пиером и рядовьм, не припержавастся строгого

12345

полового распределеяня ролей

13. Наqинает и формирует цружескате отнопгения

12345

14. Показываст положктелыное отнотение $\mathrm{x}$ взростыл, баланс меххиу занистисстью! частитной завистмостью/

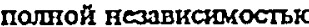

15. Дехонетрирует терпимость,

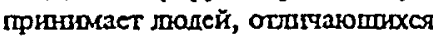

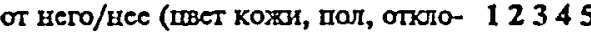

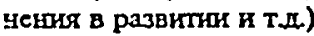

\section{ЯЗЫК/ОБЩЕНКЕ}

16. Слушает и вритымгает активное участие в беседах н дискууссиях во 12345

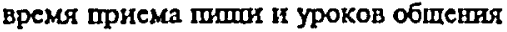

17. hastaraet orнсание собитй и собствентопо отыта ЛОГично н последовательно

\section{5}

18. Полугает удовопьствие запомппая и распевая песин

12345

19. Строит свос общение так, что сro/ec пономанот окружаюшие

\section{5}

ДВИТАТЕЛЬНЫЕ КАЧЕСТВА

20. Легко привлекзетоя и активно учавствует во всех гоитательныв/ моторнах винах деятельности /рисование, составленис мозанк, вырезание и т.I/

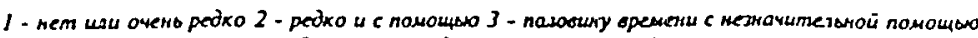

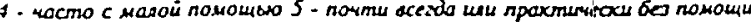


21. Летко и свободны беraer, прытает, лазает, езлит и приседает без qастьх 12345 падендй и потерь равновесия.

22. Охотно проверяет свою ситу и Возмо,жНОСТЯ

\section{5}

РАСКЛАДКА ВИДОВ ДЕЯТЕЛЬНОСТИ

Приғарает участие и получает удовољьствис от следуюших созидательньб занятий:

23. Изобразитељьнсе исскуаство

12345

24. Драма/театр/предстаяленнс

12345

25. Дандение : $\quad 12345$

20. Проявление лобознатетьности

12345

27. Kuгет на риск, и в то зве времл не выхолит 32 рамка безопасности свосй и. друтих

ПОЗНАВАТЕЛЬНАЯ ДЕЯТЕЛЬНОСТЬ/РЕПТНИЕ ПРОБЛЕМ

28. Преодолевает дерьсзные препятствия на пути решения задачи, натример в составлении сложнњх 12345 конструкпий и рсшенпй при сложенти составнғах картннок.

29. Во время выббора вида выбирает и продолокаст зандматься видом деятельности десльь минут и более. 12345

30. Проявляет многосторонний попход к решенпюо задач, может предіожыть нескопько вариантов выхода из создавщейся ситуашин.

1 - нер или очень редхо 2 - редко и с помоцьо 3 - половшу аремени с незночительной помощью 4 - часто с малой понощоко 5 - почти өсеzda ияи практически бе помощи. 
ПОЗНАВАТЕЛЬНАЯ ДЕЯТЕЛЬНОСТЬ - МАТЕМАТИКА

В итре с таками предметами как книти, палочки, тарелки и т.д.

31. Разделение предметов по признаку /голубыс карточки от краснбых/

12345

32. Количество /подсчет предометов/ 12345

ПОЗНАВАТЕЛЬНАЯ ДЕЯТЕЛЬНОСТЬ/ ГРАМОТНОСТЬ

33. Стуласт расскаг от начала до конша вниматељьо

\section{5}

34. Проявляет интерес к "печатному

слову", рассматривает кант, спрашпиаст о значенги знаков и символов и т.д.

12345

35. Пишет, рисует, создает тто-либо представляя себя, поцписьвает работы, имитнрует написание рассказа в течение сценки ити представления, рисует изображения известнбх людей й объекТов.

\section{5}

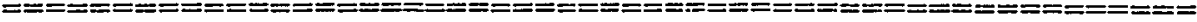

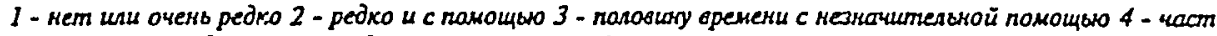
малой помощью 5 - почти өсегда иаи прахтикески без помощи.

Другие замечания и комментария: 


\section{Oregon Evaluación para niños de 3-5 años en clases para desarrollo apropiado}




\section{Oregon Evaluación para niños de 3 a 5 años en clases para desarrollo apropiado Versión 1.4 agosto 1992}

Fondo. Este documento se desarrollb por los coordinadores de educaclón de Oregon Head Start en noviembre de 1989. El proyecto fue concebldo, dirigido. y preparado por Steffen Saifer del Early Childhood Training Center, School of Extended Studies, Portland State University, P.0 Box 1491, Pon OR 97207. (503) 725-4815. El Early Childhood Tralning Center tiene el derecho de autor y es el publicador.

Propóslto - Esta evaluación fue desarrollada para que los(as) maestros(as) se enfoquen en los comportamientos y abilldades que son los mas import para el crecimiento sano y desarrollo de rinos preescolares. Por lo tanto, hay un éntasis mayor en autoconcepto, abilidades soclales, comportamientt juego, actitudes, y disposiciónes y hay menos énfasis en la ejecución de abilidades. Temas son escritas para que maestros (as) puedan lácilmente he metas apropladas para ninos. La Oregon Evaluación provee información pertinente y útil sobre nlhos (y su progreso) para ser sompartida con padres otros profesionales. La Oregon Evaluaclén no es para seleccionar nl diagnosticar niños. Es una herramlenta para: determinar que tan blén funcionar en clases para desarrollo apropiado, planificación de currículum, seguir el progreso de nihos, proveer información adicional a profesionales cuando se un nirio. y conferenclas con padres.

Notas - Haga un clrculo alrededor del numero que mas describe el comportamiento o abilldad del nino: 1 - Lo hace poco o nunca 2 - Lo hace de vez cuando o con mucha ayuda 3 - Lo hace casi la mitad del tiempo 4 - Lo hace segido o con poca ayuda 5 - Lo hace casl slempre o sin ayuda. Solo er tema 'Procesos Mentales -Matemática' se hacen las notas de una manera distinta, como se muestra en la página 5 . Se debe usar segido el área 'comentario/ejemplos'. La evaluaclón se debe hacer una vez en la parte temprana del ańo programado (o pronto despú́s de que un niño enire al programa) y entonces de nu u hacla los frion 作 negro, evaluación segunda - lapicero o lapiz rojo, evaluación tercera • lapicero o lapiz verde. Todos temas se deben evaluar por observar ai nino dura
clases, rutinas, y actividades regulares.

Formularlo de Observaclón. Se cebe usar este formulario junto con la evaluación para apoyar y aumentarlo. Se escriben relatos segldo (por lo men cada mez por cada niño) de abilidades y comportamientos observados en las clases. Escriba relatos en $2^{\circ}$ por $3^{\prime}$ "Post it Notes' y pegelos al Formule Obsera cada área del formulano). Esto ningún relato(s) en otras áreas porque se enfocarán mas observaciones en las áreas de inquietud. Lo siquiente es un ejemplo:

Ana es una nina que evita actividades de movimiento grandes porque tiene dificultad con el equilibrio y agilidad (ella saca un ' 1 ' en temas 34 y 35). La maestra hace varias metas para ella para que pueda tener exito y experiencias positivas nilentras hace actividades de movimlento grandes. Entonces la maestra hizo relatos en 'Post it Notes' mientras la observaba durante actividades planificadas, disefiadas para alcanzar aquellas metas. Después del 'Area de Desarrollo" en el Formulario de Ouservación, la maestra escribló "Movimlento' y pegó los recados por debajo. Uno de los recados dijo: 2/10 - Ana cruzó la escalera horizontal dos veces colgada con sus manos mientras me paraba al lado de ella. Ayudamos dandole aplausos Lisa y yo. Una colchoneta bajo la escalera la hlzo sentir mas segura. Veu \#34, 35.

Indlce .

\begin{tabular}{|c|c|}
\hline Página & \# de Tema \\
\hline 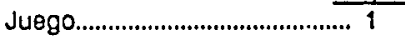 & 1.5 \\
\hline Autosuficiencia........................... 1 & 6.8 \\
\hline Autoconcepto.......................... 2 & 9.20 \\
\hline 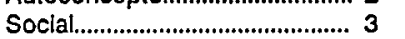 & $21-27$ \\
\hline Languaje/Comunicación................3 & 2833 \\
\hline Movimlento...........................4 & 3436 \\
\hline
\end{tabular}

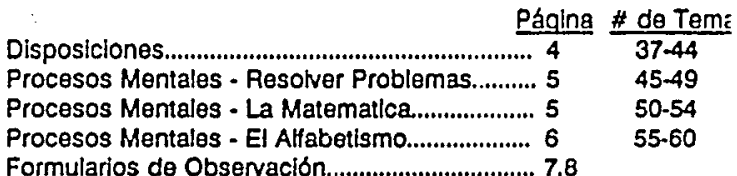




\section{Oregon Evaluación para nlños de 3-5 años en}

clases para desarrollo aproplado

\section{Nombre: $\quad$ Fecha de nacimiento:}

Programa: Maestros(as):

\section{JUEGO}

1. Participa in juego socio-dramatico con companeros(as) durante juego libre.

FECHAA Y COMENTARIO/EJEMPLOS

2. Participa activamente en muchos distintos

tipos de juego - cubos (tablitas), juego socio.

dramático, manipulattvos, Juegos de mesa, etc

3. Es autodirigido(a) y motwado(a) para
aprender - demuestra iniclattva para encontrar

4. Guarda los materiales enlugares propios cuando termina.

12345

12345

12345

5. Usa abilidades de lenguaje para ayudar a

12345

dirigir el luego.

\section{AUTOSUFICIENCIA}

6. Culda sus necesidades personales - se lava las manos, cepilla los dientes, se viste a s! mismo(a) a un nivel apropiado

7. Quiere ayudarse a si mismo(a) y esta orqulloso(a) al hacerio.

8. Cuida la segurida personal - usa el cinturón

en el carro, no es Imprudente en el campo de

juegos, nl en la clase, nj en excursiones.

1-teramento o nunca 2-esuelto o con muetro ar

Negro - Primero evaluaclo

12345

12345

12345

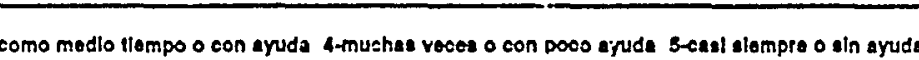

Rojo-Segundo evaluacion Verde. Tercero evaluacion 
9. Confia en adultos quienes son rasonablemente

12345

consistentes y que dan apove

12345

10. Acepta consualo de otros.

12345

11. Hace contacto, ojo a ojo, presumiendo que esto es una norma cultural.

12345

12. No es demasiado(a) amigable con personas

12345

desconocidas.

12345

13. Pide ayuda de adultos y companeros(as)

verbalmente, cortésmerite, y cuando

verdaderamente la necesita.

14. Puede hacar turnos de actwidad con otros

(Dospone gratificación por un corto plazo).

12345

15. Acepta responsibllidades por sus proplas

12345

acciones.

16. Se defiende a sl mismo(a) verbalmente con confianza cuando se mete en confilictos con otros

17. Atraviesa facilmente transiciónes entre actividades diversas.

\section{5}

12345

18. Tlene un sentido realista de sus proplas abllidades, fuerzas, y debilldades.

12345

19. Puede evaluar sus proplas acclones o

12345

comportamientos sin iuzgarse mus duro ni muy faci

20. Respeta la autoridad adulta y reglas de clase pero puede imponer su personalidad, ideas, y opiniones.

\section{5}

12345

1.raramento o nunca 2-suatio o con mucho ayuda 3-como modio tlempo o con ayuda 4-muehaz veces o con poco ayuda 5-easl alempro o sin ayuda

Hegro- Prinerocevaluaclón Rojo- segundoevaluactón verde rercerocevaluaclón 
SOCIAL

12345

FECHA Y COMENTARIO/EJEMPLOS

21. Trabaja y juega con una variedad de niños.

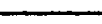

22. Hace negociaciones con otros con éxito.

12345

23. Demuestra flexibilidad al hacet papeles - puede

ser un lider y un seguidor(a) y no queda bién fijo a un

comportamiento estereotlpico de caulquier sexo.

12345

12345

necesidades con otros en su momento.

25. Hace amistades y las mantiene.

12345

26. Demuestra relaciones positivas con adultos 12345

incluso un equilibrio de dependencial

interdependencia/independencia.

27. No demuestra prejuicio hacla gente

distinta de el/ella (descapacidades,

género, color de piel, etc.)

LANGUAJE/COMUNICACIÓN

28. Escucha y contlbuye en discusiones del grupo

a la hora de comida y a la hora de compartir.

29. Cuenta sus experiencias o eventos

simples en una secuencia lógica.

30. Le gusta hacer canciones y poemas

infantiles dramáticos.

FEHA Y COMENTARIOTES

31. Se comunica para darse a entender.

12345

32 . Responde a la comunicacion de otros.

33. Empieza y sostiene conversaciones. 12345

1-rastmente o nunce 2-suetto o con mucho ayuda 3-como medlo tlempo o con ayude 4-muchas veces o con poco ayuda 5-casl alempre o aln ayude

Hegro-Primeroevalueción Rojo-segundoevaluación verde- Terecroevaluactión 
MOVIMIENTO

34. Participa activamente y goza de actividades

coñ sus manos-(debujai, -pintar,-fompocabezas,

conar, elc.)

35. Libremente corre, salta, trepa, maneja sin caerse ni desequilibrarse.

36. Se desalia voluntariamente de acuerdo a

fuerza propla y agilidad (movimiento fino

y/o bruscol.

DISPOSICIONES

Paricipa en y goza de actividades creativas y expresión como las siguientes:

37. Resuelve creativamente problemas. 12345

\begin{tabular}{ll}
\hline 38. El arte & 12345
\end{tabular}

38. El arte

12345

39. La dramática $\quad 12345$

40. El movimiento $\quad 12345$

41. La música 12345

4. La musica

42. Demuestra curiosidad.

12345

43. Voluntariamente trata de

12345

hacer actividades nuevas.

12345

44. Le gusta tomar riesgos mientras mantiene

sequridad de si mismo y de otros.

FECHA Y COMENTARIO/EJEMPLOS

ENTARIO/EJEMP

1.raramente o nunca 2-suotto o con micho ayuda 3-como medio tiempo o con ayude 4-muches rocos o con poco eyuda E-cesl siompro o sin ayuda Hegro. Primeroevaluactón Rojo. Segundoevaluación verde. Tercerocvaluación 
45. Hace preguntas: ¿¿Como,por que, dónde, .

12345

cuando, qulen?: a adultos y niños

\section{5}

46. Intenta tareas con conflianza

no dice "No puedo"

12345

47. Es persistente en tareas difficiles a pesar

de retrasos como rompocabezas dificiles o

construcciones de cubos 'Lega'.

48. Escoge active durante juegos libres y

12345

puede persistir por 10 minutos o mas.

49. Demuestra habilidades cuando encuentra problemas u obstaculos - pueje creanarias problemas u obstaculos - pueve creanarias
soluciones/alternativas cuando le hacen

prequntas como "QQue mas puedes hace: ?.

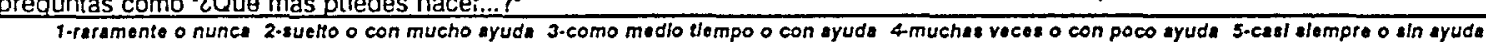

12345

PROCESOS MENTALES - LA MATEMÁTICA*

Cuando juega con cosas en el ambiente como cubos, clavijas, platos, etc.:

50. Empareja (i.e.; carros rojos en caja roja)

12345

51. Clasifica (l.e.; carros

rojos de carros azules)

12345

52. Arregla en serie (i.e.; hace cola de carros

desdeel mas pequeño hasta el masgrande

12345

53. Secuencias (i.e.; carro rojo, carro azul, carro rojo,carro azul)

12345

54. Cuantifica (t.e.; cuenta 5 carros)

12345

"Para Proceso Mentalos - Le Matemíltca: 1-nunce 2-muy minlmo hebllidad o con mucho ayude 3-medio habllided o con aysda 4heblldad o con poco ayude 5-mucho habilidad o sin ayuda 


\section{PROCESOS MENTALES - EL ALFABETISMO}

55. Escucha un cuento entero

disrante la hora de cuentos.

56. Pide que se le lea

12345

57. Demuestra interés en cosas imprentas - le

gustamirar libros, pregunta que dicen

$$
\text { los rótulos ete }
$$

58. Reconoce y nombra letras en su ambiente:

imprentas, o cuando se dictan nombres de

59. Lee su propio nombre en dibujos, tablas, étc. v lee-rouitios simples on su ambiente.

60. Escribe, dibuja, y/o hace cosas con forma representacional, i.e; escribe su nombre en

arte, imita al escribir durante juegos dramáticos,

hace dibuios que son cosas o gente reconocibles.

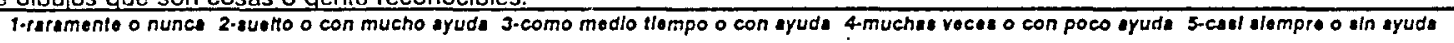

Negro. Primero evaluación

Orros Comentarios o Observaciones:

\section{FECHA Y COMENTARIO/EJEMPLOS}

12345

12345

12345

12345 
OREgON EVALUACION PARA NINOS DE 3 A 5 ANOS EN CLASEPARA DESARROLLO APROPLDO FORMULARYO DE ORSERVACTON

Nombre:

Ponga fecha

de todo comentario

Area de desarrollox 
APPENDIX E

VAIIDITY SURVEY 
P.O. Box 1491 Portand, OR 97207.1491

October 29, 1993

I would greatly appreciate your help by completing the enclosed survey. You have been selected as a well respected early childhood educator with great knowledge and expertise. I highly value your opinions.

This survey is one part of my doctoral dissertation research to determine validity, reliability and usefulness of the Oregon Assessment. The survey will provide information regarding construct, curricular, and item validity. Congruent validity, interrater reliability and test-retest reliability will be determined by research involving the testing of a diverse group of children. Usefulness will be determined with a different survey sent to a diverse group of current users (teachers and administrators).

Enclosed is a copy of the assessment and a copy of the User Manual. If you have the time, reading the first 8 pages of User Manual will give helpful background information regarding the development of the assessment and its intended purposes. The rest of the manual focuses on interpreting and scoring the 60 items in ten domains (play, selfhelp, self-concept, social, language/communication, motor, dispositions, cognitiveproblem solving, cognitive-math, cognitive-literacy).

Please use the manual as you complete the survey to help you determine what each item asks for and how to interpret the item. (Item explanations begin on the bottom of page 8 of the manual).

I know you are busy and your time is precious, so I am deeply grateful for your assistance on this project. Feel free to call if you have any questions - 503 - 725-4835. (or 1-800-547-8887 x4835). Please return the survey by November 24.

Sincerely

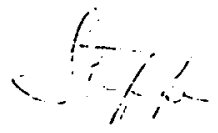

Steffen Saifer

Early Childhood Education Specialist 
Please circle the number that best describes how adequately each item -

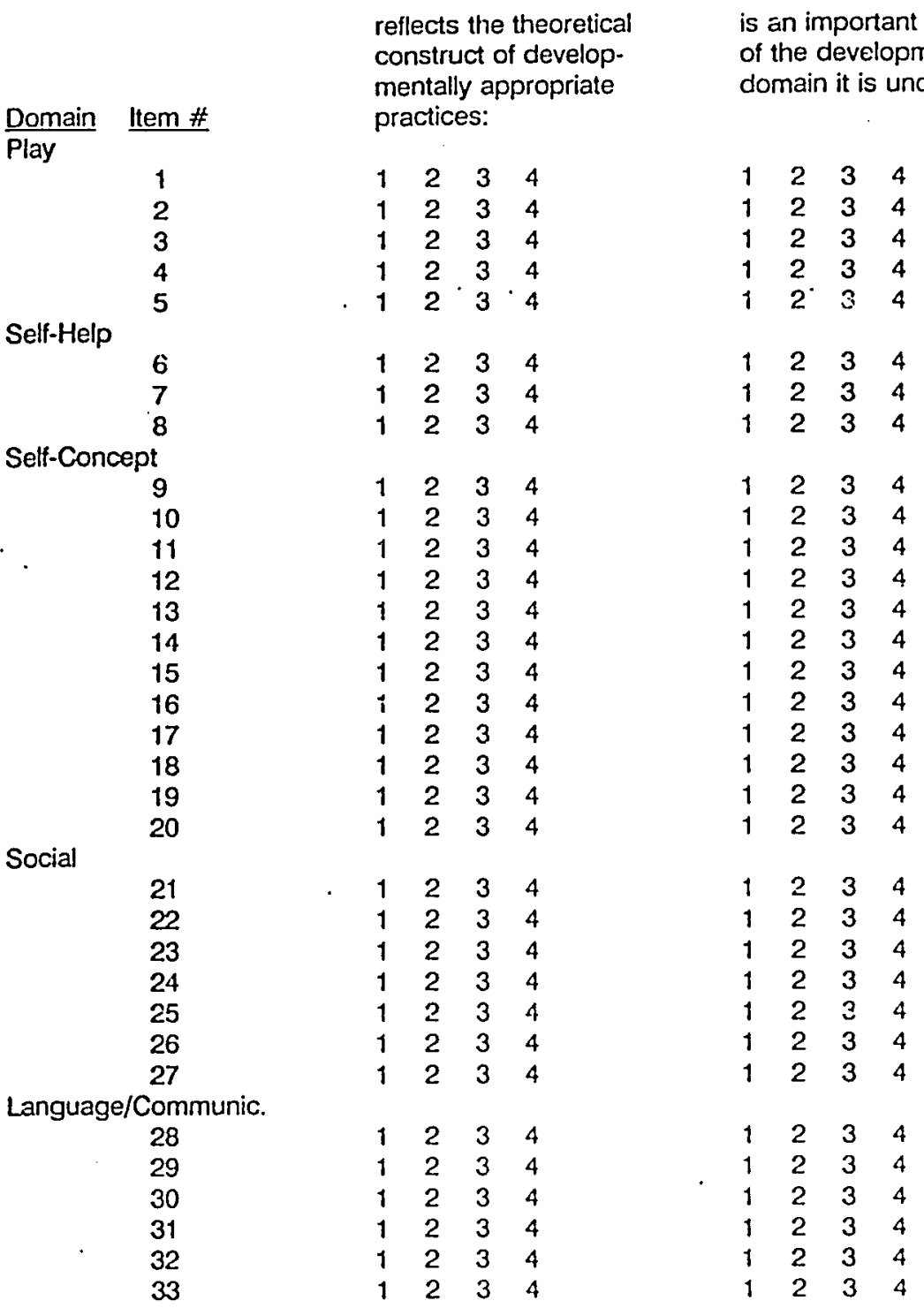

$1=$ not at all, $2=$ a little, $3=$ generally, $4=$ strongly

items continued on next page 
Please circle the number that best describes how adequately each item -

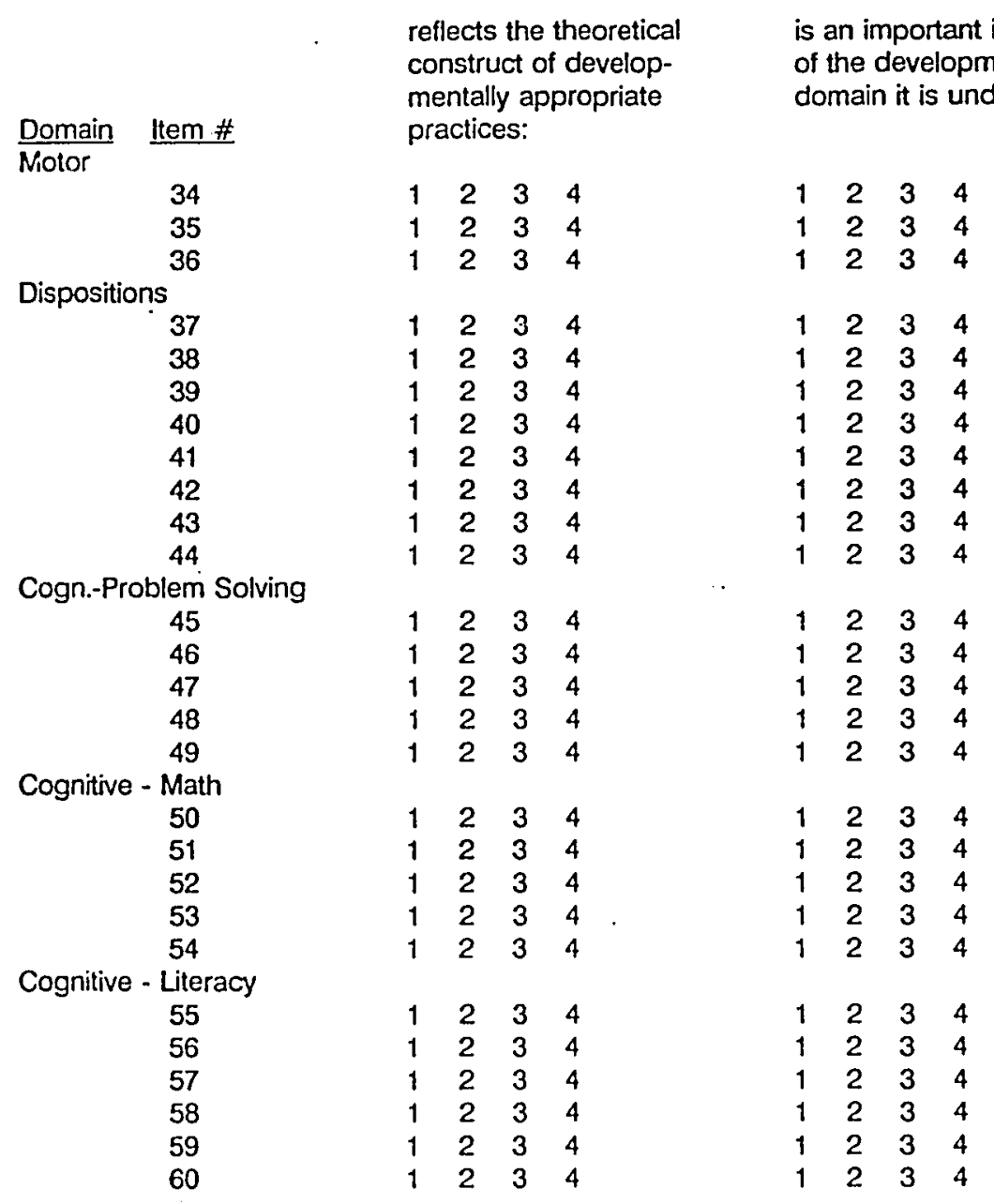

$1=$ not at all, $2=$ a little, $3=$ generally, $4=$ strongly 
Please circle the number that best describes how adequately each domain reflects the theoretical construct of developmentally appropriate practices:

\section{Domain}

Play

Selt-Help

Self-Concept

Social

Language/Communic

Motor

Dispositions

Cogn.-Problem Solving

Cognitive-Math

Cognitive-Literacy $\begin{array}{llll}1 & 2 & 3 & 4\end{array}$

$\begin{array}{llll}1 & 2 & 3 & 4\end{array}$

$\begin{array}{llll}1 & 2 & 3 & 4\end{array}$

$\begin{array}{llll}1 & 2 & 3 & 4\end{array}$

$\begin{array}{llll}1 & 2 & 3 & 4\end{array}$

$\begin{array}{llll}1 & 2 & 3 & 4\end{array}$

$\begin{array}{llll}1 & 2 & 3 & 4\end{array}$

1. 234

$\begin{array}{llll}1 & 2 & 3 & 4\end{array}$

$\begin{array}{llll}1 & 2 & 3 & 4\end{array}$

Please circle the number that best describes how adequately all domains taken together as whole reflects the theoretical construct of developmentally appropriate practices

$$
\begin{array}{llll}
1 & 2 & 3 & 4
\end{array}
$$

Please circle the number that best describes how adequately this assessment as a whole reflects principles of appropriate assessment as outlined in the 1991 NAEYC position statement: Guidelines for appropriate curriculum content and assessmient in programs serving children ages 3 through 8 (Young Children, 46[3], 21-38) (article attached):

$$
\begin{array}{llll}
1 & 2 & 3 & 4
\end{array}
$$

$1=$ not at all, $2=$ a little, $3=$ generally, $4=$ strongly

Please write any additional comments you have below (continue on the back if needed): 
for planning instruction and communicating with parents. Guidelines for screening and program evaluation follow. (For additional information on the topic of assessment, see also NAEYCs Position Statement on Standardized Testing of Young Children (NAEYC. 1988) and Unacceptable Trends in Kindergarten Entry and Placement (NAECSSDE. 1987), and Kamii (1990).)

Guidelines for planning instruction and communicating with parenis. Assessment of children's development and learning is absolutely necessary if teachers are to provide curriculum and instruction that is both age-appropriate and individually appropriate. An initial assessment is necessary for teachers to get to know children and to adjust the planned curriculum. The appropriate use of initial assessment is to find out what children already know and are able to do and to use this intormation to adjust the curriculum to the individual children. Too often, initial assess. ment takes the form of "readiness test ing" with young children or "achievement testing" with older children. the results of which are used to exclude children Irom the program, track them by ability. or otherwise label them. How the initial assessment is conducted will deternine the accuracy and usefulness of the findings. To provide an accurate picture of children's capabilities, teachers must observe children over time; information obtained on one briel encounter may be incomplete or distorted. Likewise, initial assessment information must be used to adjust curiculum and instruclion. If assessment data are ignored and no adjustments are made, then the data should not be collected. Moreover, assessinent data should be used to bring about benefits lor children such as more individualized instruction; it should not be used to recommend that children stay out of a program. be retained in grade, or be assigned to a segregated group based on ability or develop. mental malurity.

The following principles should guide assessment procedures tor chil. dren ages 3 through 8

1. Curriculuin and assessment are integrated througliout the program, as sessmellt is congruent with and rel. evant to the goals, objectives, and con tent of the program.

2. Assessment results in benelits to the child such as needed adjustments in the curriculum or more individualized instruction and improvements in the program

3. Children's development and learning in all the domains-physical, social emotional, and cognitive-and their dispositions and leelings are informally and routinely assessed by teachers observing children's activitites and interactions, listening to them as they talk, and using children's constructive errors to understand their learning

4. Assessment provides teachers with useful information to successfully lulfill. their responsibilities: to support chil dren's learning and development, to plan lor individuals and groups, and to communicate with parents.

5. Assessment involves regular and pericdic observation of the child in a wide variety of circumstances that are representative of the child's behavior in the program over time.

6. Assessment relies primarily on procedures that reflect the ongoing lite of the classroom and typical activities of the children. Assessment avoids approaches that place children in artificial situations. impede the usual learning and developmental experiences in the classroom, or divert children from their natural learning processes.

7. Assessment relies on demonstrated performance, during real, not contriver activities, for example, real reading and writing activities rather than only skills testing (Engel, 1990; Teale, 1988).

8. Assessment utilizes an array of tools and a variety ui prccesses including but not limited to collections of repre sentative work by children (artwork. stories they write. tape recordings of their reading). records of systernatic observations by teachers. records of conversations and interviews with children, teachers summaries of chil dren's progress as individuals and as groups (Chittenden \& Courtney. 1989. Goodman. Goodman. \& Hood. 1989)

9. Assessment recognizes individual diversity of learners and allew's tor dil lerences in styles and tale's of fearmm! Assessment hikes into consuderatmo
The following guidelines first ad dress the priniary use of assessmient 
children's ability in English. Uheir stage of language acquisition, and whether they have been given vie time and opportunity to develop proficiency in their native language as well as in English.

10. Assessment supports children's de. velopment and learning; it does not threaten children's psychological salety or feelings of sell-esteem.

11. Assessment supports parents' relalionships with their children and does not undermine parents' confidence in their children's or their own ability, nor does it devalue the language and culture of the tamily.

12. Assessment demonsträtes children's overali strengtis ani progress. what children can do, not just their wrong answers or what they cannot do or do not know.

13. Assessment is an essential component of itic teacher's role. Since teachers can make maximal use of assessment results, the teacher is the primary assessor.

14. Assessment is a collaborative process involving children and teachers, leachers and parents, school and community. Inlormation from parents about exch child's experiences at home is used in planning instruction and evaluating children's learning. Information obtained from assessment is shared with parents in language they can understand.

15. Assessment encourages children to participate in self-evaluation.

16. Assessment addresses utiat children can do independently and what they can demonstrate with assistance. since the latter shows the direction of their growth.

17. Inlormation about each child's growth, development, and learning is systematically collected and recorded at regular intervals. Information such as samples of children's work, descrip tions of their pesformance, and anec dotal records is used lot planning in struction and communicating with parents.

18. A regular process exists for periodic inlormation sharing between teachers and parents about childrenis growith and development and perform ance. The nethod of reporting to pat ents doe's not rely on benter or nument

roung Clutdern * Musch Isyl cal grades, but rather provides more meaningtul, descriptive inlormation in narrative form.

Questions to ask in evaluating a program's assessment procedures

1. Is the assessment procedure based on the goals and objectives of the spe cific curriculum used in the program?

2. Are the results of assessment used to benefit children, ie to plan for individual children, improve instruction, identify children's interests and needs. and individualize instruction, rather Itan labcl, racke or tail childrai?

3. Does the assessment ptocedure address all domains of learning and development-social, emotional. physical, and cognitive-as well as children's Icelings and dispositions toward learning?

4. Does assessment provide uselut in formation to teachers to help them do a better job?

5. Does the assessment procedure rely on teachers" regular and periodic observations and record-keeping of chitdren's everyday activities and perform ance so that results reflect children's behavior over time?

6. Does the assessment procedure $\propto$ cur as part of the ongoing life of the classroom rather than in an artificial. contrived context?

7. Is the assessment procedure performance-based, rather than only testing skills in isolation?

8. Does the assessment rely on multiple sources of information about children such as collections of their work. results of leacher interviews and dia logues, as well as observations?

9. Does the assessment procedure tellect individual. cultural, and linguistic diversity? Is it tree of cultural, language. and gendet biases?

10. Do children appear comfortable and relaxed during assessment rather than tense or anxious?

11. Does the assessment procedure support parents conlidence in their children and their ability as parents rather than threaten or undermine par. nits confidence?

2. lowes the assessnent examine chil drenis suengullus and capabitities rather than fust theit weatinesses of willat

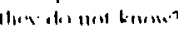

13. Is the teactice the primary assessor and are teachers adequately trained for this role?

14. Does the assessment procedure in volve collaboration among teachers. children, administrators, and parents? Is intormation trom parents used in planning instruction and evaluating children's leaming? Arc parents in. cormed about assessment information?

15. Do children have an opportunity to reflect on and evaluate their own learring?

16. Are children assessed in supportive contexts to determine what they are capable of doing with assistance as well as what they can do indipendently?

17. Is there a systematic procedure for collecting assessment data that laciliutes its use in planning instruction and communicating with parents?

18. Is there a regular procedure for comnunicating the results of assessment to parents in meaninglul lariguage, rather than letter or number grades, that reports children's individual progress? 
APPENDIX F

UTILITY SURVEY 


\section{Portland State University}

PO Box 1491 Porland OR 97207-1491

\section{Dear Friend,}

1 appreciate your help in my attempt to obtain information about the usefulness of the Oregon Assessment. This is part of a research project that includes establishing reliability and validity of the assessment. It is my doctoral dissertation research and is funded by a Head Start research grant.

Please complete all items on the survey and return it in the attached envelope within a week of receiving it. It is completely confidential and you will not be identified by name.

If you have any questions please call me at 1-800-547-8887 $\times 4835$ or (503) 725-4835.

Thank you again for your help and your prompt response.

Sincerely.

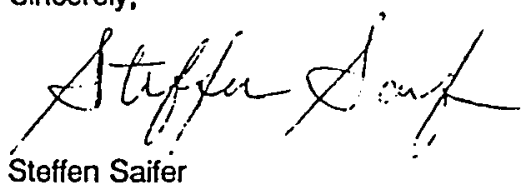

Early Childhood Education Specialist (and doctoral student!) 


\section{Usefulness of the Oregon Assessment for 3-5 Year Olds in Developmentally Appropriate Classrooms}

Please circle the number that best describes your response to each statement:

1=Strongly Disagree, 2=Disagree 3=Agree 4=Strongly Agree

1. The results from the Oregon Assessment accurately reflect the skills, behaviors, and abilities of my children.

$$
\begin{array}{llll}
1 & 2 & 3 & 4
\end{array}
$$

2. The Oregon Assessment includes the most important behaviors to assess in children.

$$
\begin{array}{llll}
1 & 2 & 3 & 4
\end{array}
$$

3. The criteria for the items as presented in the User Manual are clear.

$$
\begin{array}{llll}
1 & 2 & 3 & 4
\end{array}
$$

4. The scoring system as explained in the User Manual is understandable.

$$
\begin{array}{llll}
1 & 2 & 3 & 4
\end{array}
$$

5. The Oregon Assessment is effective for helping me write goals for children.

$$
\begin{array}{llll}
1 & 2 & 3 & 4
\end{array}
$$

6. For individualized curriculum planning and tracking children's progress the Oregon Assessment is an effective tool for me.

$$
\begin{array}{llll}
1 & 2 & 3 & 4
\end{array}
$$

7. The Oregon Assessment is more useful than other assessment tools I have used for individualized curriculum planining and tracking children's progiess.

$$
\begin{array}{llll}
1 & 2 & 3 & 4
\end{array}
$$

8. Parents respond favorably to the Oregon Assessment.

$$
\begin{array}{llll}
1 & 2 & 3 & 4
\end{array}
$$

9. The Oregon Assessment is useful for helping parents understand their children and set appropriate goals for them.

$\begin{array}{llll}1 & 2 & 3 & 4\end{array}$

(please go on to the back) 


\section{$1=$ Strongly Disagree, $2=$ Disagree $3=$ Agree $4=$ Strongly Agree}

10. The assessment is responsive to the diversity (ethnic, cultural, ability, gender, etc.) of my children and families.

$$
\begin{array}{llll}
1 & 2 & 3 & 4
\end{array}
$$

11. The completed assessment provides a rich and in-depth "picture" of a child.

$$
\begin{array}{llll}
1 & 2 & 3 & 4
\end{array}
$$

12. The average amount of time it takes to complete the assessment on each child is:

$$
\text { Hours }
$$$$
\text { Mirutes }
$$

13. I typically complete the assessment on one child over a period of days.

14. It takes a reasonable amount of time to complete the assessment.

$$
\begin{array}{llll}
1 & 2 & 3 & 4
\end{array}
$$

15. The total amount of training on using the Oregon Assessment I received was (check one):

[ ]none [ ]less then 3 hours [ ] 3-4 hours [ ]5-7 hours। [ ]other If you checked other, write the number of hours here

16. I am a (check one): [ ]Head Teacher [ ]Assistant Teacher [ ]Ed Supervisor or Coordinator [ ]Other

17. I have been using the assessment for years months.

18. The highest degree I have earned is (check one): [ ]none []CDA []AA []BABS []MAMS []Other

The number years I have been teaching preschool is (check| one): [ ]less than 1 [ ]1-2 [ ]3-4 [ ]5-6 [ ]7-10 []11-15 []16-20 [ ]more than 20

Please write any additional comments you have below. Use an additional page if more space is needed. 
APPENDIX G

THE OREGON ASSESSMENT FOR 3-5 YEAR OLDS IN DEVELOPMENTALLY APPROPRIATE CLASSROOMS: SHORT VERSION 


\title{
OREGON ASSESSMENT FOR 3-5 YEAR OLDS IN DEVELOPMENTALLY APPROPRIATE CLASSROOMS
}

\author{
SHORT VERSION (35 ITEMS) \\ Version 1.5 August 1993 \\ Early Childhood Training Center \\ Portland State University \\ School of Extended Studies \\ P.O. Box 1491 \\ Portland, OR 97207
}

(503) 725-4815 


\title{
๑) 1990 Early Childhood Training Center All Rights Reserved
}

\author{
Published and Printed by the Early Childhood Training Center \\ School of Extended Studies \\ Portland State University \\ P.O. Box 1491, 1633 SW Park \\ Portland, OR 97207
}

Use Restrlctions and Prlclng - Any photocopying of this Instrument (all or part) is strictly prohibited unloss done so by a stafl member of any of the following Oregon Head Start progrems (only for use within these programs): Columbia Pacific, Curry County, Deschutes/Crook Co.

Eastern Oregon, Kidco, Klamath Family, Malhour Co, Mld-Columbla, Portland Public Schools - ECE, Southern Oregon, Umatilla/Morrow Co. Warm Springs Tribal. All other parties must purchase the Oregon Assessment for a cost of $\$ 5.00$ lor one form, $\$ 15.00$ for a package of 10 , $\$ 25.00$ for a package of 20 (add $10 \%$ or $\$ 1.00$, whicherer is greater, to the total order for postage and handling). Licensing agreements can be arranged lor large programs or school districts. A User Manual is available for $\$ 10$. Order from the address listed above. 


\section{Oregon Assessment for 3.5 Year Olds In Developmentally Approprlate Classrooms}

\section{Version 1.5 August 1993}

Background - This instrument was developed by Oregon Head Start education coordinators in November 1989. The project was conceived, directed, and cdited b Steffen Saifer of the Early Chiluhood Training Center, School of Extended Studies, Portland State University, P.O. Box 1491, Portland, OR. 97207. (503) 725.48: The Early Childhood Training Center holds the copyright and is the publisher.

Purpose - This assessment was developed so that teachers will focus on behaviors and abilities that are the most important for healthy growth and development o young children. Therefore, there is a strong emphasis on self-concept, social skills, play behaviors, attitudes and dispositionis and less emphasis on the performan of skills. Items are written so that teachers can easily set developmentally appropriate goals for children. The Oregon Assessment provides pertinent and useful information about children (and their progress) to share with parents and other professionals. The Oregon Assessment is not for screening or diagnosing childre It is a 100 for: Determining how well chlldren function in a developmentally appropriate classroom, curriculum planning, tracking children's progress, providing additional information to professionals when referring a child, and conferencing with parents.

\section{INSTRUCTIONS}

A User Manual is avallable and highly recommended. It provides detalled Information on the purpose of the tool and on interpreting and scoring items. Scoring - Circle the number that best describes the child's behavior or ability: 1 - Does it rarely or not at all 2 - Does it oceasionally or with much help 3. Does it about half the time or with some help 4 - Does it often or with little help 5 . Does it almost always or with no help. Only. Cognitive Math is scored differently as indicated on page 5. The "comments/examples" area should be used often. Assessment should be dune once in the early part of the program year ( soon after a child enters the program) and then again towards the end of the program year. A mid.year assessment is also a good idea, if possible. To keep the data from the whole year on one form per child, color code your circles and comments accordingly: 1 st assessment - Black pen or pencil, 2nd assessment . Red or pencil, 3rd assessment. Green pen or pencil. All items should be assessed through observing the child during regular classroom routines and activities.

Observation Form - This form is to be used in conjunction with the assessment to support and extend it. Anecdotes are written on an on-going basis (at lcast monthly for each child) of skills and behaviors observed in the classroom. Write anecdotes on $2^{*} \times 3^{*}$ Post-lt Notes and stick them on the observation form und the appropriate area of development (you label the area on the form). This system allows you to write aneodotes only once (no need to recopy) and allows for maximum fexibility. Most children will have more anecdotes in one or several areas of development and few or no gnerdotes in other areas because you will for more obscriations on areas of concern. The following is an example:

Anna is a child who avoids large motor activities because she has difficulty with balance and agility (she scored a " 1 " on liems 3.4 and 35). The tescher set several goals for her so that she can have some success and positive experiences doing large motor activities. The teacher then took aneodotes on Post-It Notes while obsetving her during the planned activitles designed to meet those goals. After "Area of Development" on the Observation Form, the teacher wrote 'Motor' and stuck the notes below it. One of the notes said: 2/10 - Anna twice crossed horizontal ladder hanging from her hands while I stood next to her. Helped by checring from Lisa \& me. Gym mat under-tadder made-her-fee!-more secure. See_\#34; 35 .

Table of Contents.

\begin{tabular}{|c|c|c|}
\hline \multicolumn{3}{|c|}{ Page liem \#s } \\
\hline & & 1.3 \\
\hline & & 4.5 \\
\hline & & $6 \cdot 10$ \\
\hline & & 11.15 \\
\hline e/Cornmunica & & $16-19$ \\
\hline 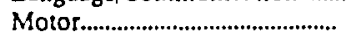 & & $20-22$ \\
\hline
\end{tabular}

\begin{tabular}{|c|c|}
\hline & \\
\hline & \\
\hline ing..... & 3 \\
\hline$\ldots$ & 3 \\
\hline iteracy........................... & 4 \\
\hline 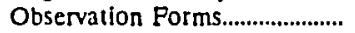 & \\
\hline
\end{tabular}


Oregon Assessment for 3-5 Year Olds in

Developmentally Approprlate Classrooms

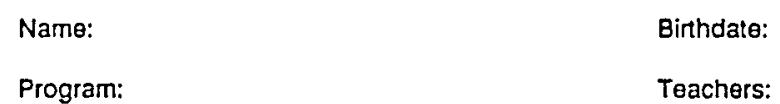

PLAY

12345

DATE COMMENTS/EXAMPLES

1. Engages in cooperative (dramatic) play

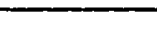

Actively engages In many different klnds of

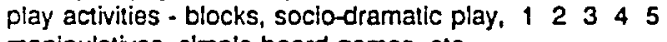

manipulatives, simple board games, etc.

3. Is a sell-directed, motivated learner -

shows initiative In finding materials

12345

or choosing activities

SELF.HELP

4. Takes care of personal grooming needs -

washes hands, brushes leeth, dresses self

5. Wants to help her/himsell and takes pride $\quad \begin{aligned} & 12345 \\ & \text { in }\end{aligned}$

in doing so.

SELF.CONCEPT

6 . Trusts adults who are reasonably

12345

consistem and supportive.

7. Requests haip from aduits and peers verbally, politely, and when actually needed

12345

8. Accepts responsibility fer own

12345

actions

9. Moves easily through transitions

12345

(such as circlo to snack)

\section{5}

. Respects adult authority and classioum

rules, but can assert own oplnions,

ideas, and personaltiy.

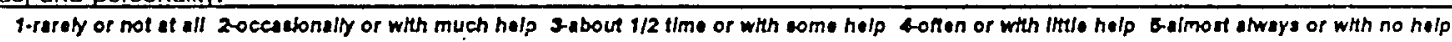

Black - tel essossment Red-2ndessossment Groon-3rdessessmens 
SOCIAL

11. Negotlates effectively

DATE COMMENTS/EXAMPLES

Displays flexibility in roles - can bo

a leader and a follower and does

not adhere

12345

13. Forms and maintains

12345

triendshlps

14. Displays postive relations with adults including a balance of dependence/ interdependence/ndependence.

15. Shows acceptance of people who are different from her/himself

(disablitities, gender, skin color, elc.)

12345

LANGUAGE/COMMUNICATION

16. Listens to and contributes to group dis-

cussions at mealtimes \& sharing ilmos.

17. Tells experiences or simple events in

12345

18. Enjoys recting fingerplays and singling songs.

19. Communicates so s/he is

understood by others

12345

MOTOR

20. Actively engages in and enjoys fine

motor acthintes (drawing, painting.

puzzles, cutting. using manipulatives)

21. Freely runs, Jumps, hops, climbs, ridts

without frequent falling or imbalance

22. Willingly challenges own strength

and aqility (fine and/or large motor)

12345

12345

12345

12345

12345

1.rarely or not al all 2 -cccaslonslly or with much help 3 about $1 / 2$ time or with some help foften or with linto holp 5 slmosi aivays or with no help 
DISPOSITIONS

Engages in and enjoys creative activites \& expression such as:
23. Ant
12345

\begin{tabular}{llll}
\hline 24. Drame & 12345 \\
\hline 25. Movement & 12345
\end{tabular}

26. Displays curlosity.

27. Enjoys takng isks whlo manting 12345

27. Enjoys taklng risks while maintalning

12345

salety of self and others.

COGNITIVE - PROBLEM SOLVING
28. Perseveres througin a difficult task
desplte setbacks such as difflcult

puzzles or lego constructions.

29. Chooses activities during free play and

can stlck with th for 10 minutes or more

30. Displays resourcefulness when faced with

problems or obstacles - can generate

several solutinns/atternatives when

asked questlons such as "What else

Can you do,..?

COGNITIVE - MATH*

When playing with objects in the environment such as blocks, pegs, dishes, etc.:

31. Sorts (i.e.; red cars from

12345

blue cars)

32. Quantfies (l.e.; counts

12345

12345

12345

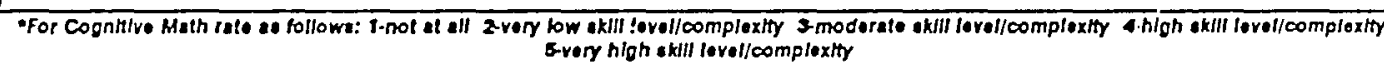

Black- tst assossment Red-2nd acsosument Greon-3ro assessment 
COGNITIVE - LITERACY

33. Listens to entire story

during stongime.

34. Shows Interest in print medium enjoys looking at books, asks

35. Writes, draws, and/or makes things repre

sentationally, l.e.; writes name on ent-

work, Imhates writing during dramatic

play, draws pictures that are recog.

nizable objects or people.

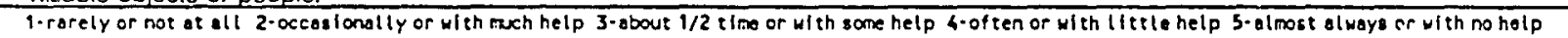

Bleck - tst assessmont Rod-2nd estossment Groan-ard assessmont

Other comments or observatlons:
12345

12345

12345 
Area of development: 
APPENDIX $\mathrm{H}$

"ONE PAGE" FORMATS FOR THE FULL VERSION AND SHORT VERSION OF THE ASSESSMENT 


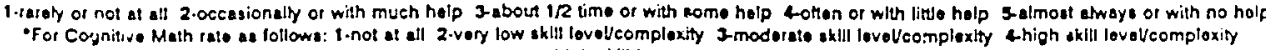

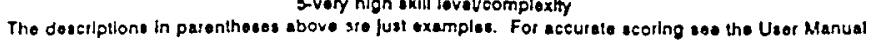

Name:

\begin{tabular}{|c|c|}
\hline 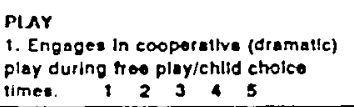 & 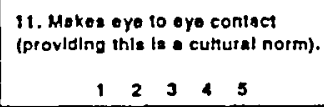 \\
\hline 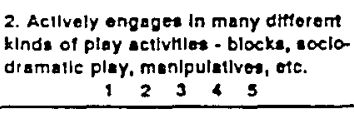 & $\begin{array}{l}\text { 12. Showe eppropilate cautlon } \\
\text { with strengers } \\
12345\end{array}$ \\
\hline 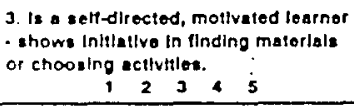 & $\begin{array}{l}\text { 13. Requeste help from aduha } \\
\text { and peors verbally, polthely, and } \\
\text { when setually nooded. }\end{array}$ \\
\hline $\begin{array}{l}\text { 6. Puls malerlals in proper piace } \\
\text { when finishod. } \\
\qquad 1233,5\end{array}$ & $\begin{array}{l}\text { 14. Can iake turns with othors } \\
\text { (delay gratincallon for a stort } \\
\text { perlod). }\end{array}$ \\
\hline 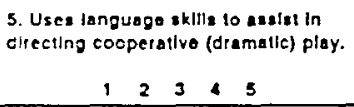 & 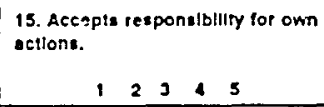 \\
\hline 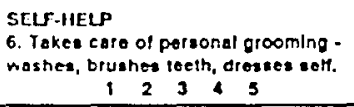 & 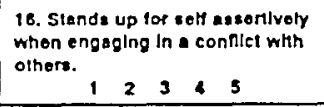 \\
\hline 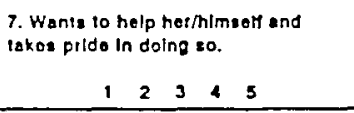 & $\begin{array}{l}\text { 17. Moves oaslly through thanst. } \\
\text { tlona (such as cliclo to enack). } \\
1\end{array}$ \\
\hline 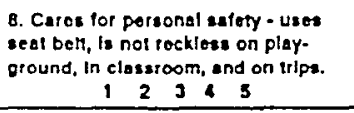 & 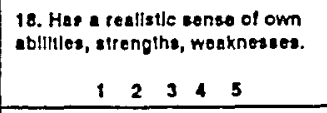 \\
\hline 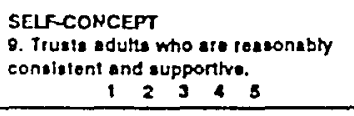 & 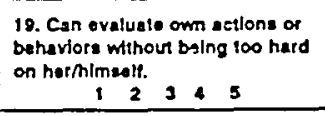 \\
\hline $\begin{array}{l}\text { 10. Actepts comtort thom othere. } \\
1123,5\end{array}$ & 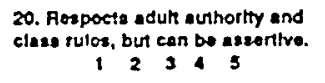 \\
\hline
\end{tabular}

Oregon Azsessment for 3.5 Yeas Olds In Developmentally Approprime Classinoms

Proprem:

\begin{tabular}{|c|c|c|c|}
\hline 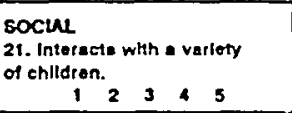 & 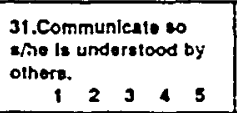 & $\begin{array}{l}12 \text { Dieptary } \\
\text { eurbotity. } \\
\begin{array}{ll}12 & 2\end{array}\end{array}$ & $\begin{array}{l}\text { 51. Sorns (1.0.; rod cars from } \\
\text { blue cars) }\end{array}$ \\
\hline $\begin{array}{l}22 \text { Nogollatios oftectively } \\
\text { wtth others. } \\
\qquad 2,345\end{array}$ & $\begin{array}{l}32 \text { Rmponsa to } \\
\text { communieatlon from } \\
\text { others. } \\
1234\end{array}$ & $\begin{array}{l}\text { 13. le milling to tiy } \\
\text { now sethtilos. } \\
123,45\end{array}$ & $\begin{array}{l}52 \text { Sorlatere (0.8.; linos up cats } \\
\text { smalleat to largorit) } \\
12345\end{array}$ \\
\hline 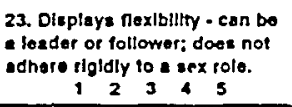 & $\begin{array}{l}\text { 23. Inhleses \& suatains } \\
\text { conversetions. } \\
12,4.5\end{array}$ & 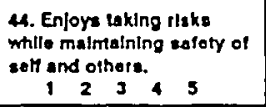 & $\begin{array}{l}\text { 53. Parturna r.o; red car, blue our, } \\
\text { rod car, biue cart) } \\
\begin{array}{llll}1 & 2 & 3 & 4\end{array}\end{array}$ \\
\hline $\begin{array}{l}\text { 24. Varbally expresses } \\
\text { roollings and noods during } \\
\text { Intoretlons with othere. }\end{array}$ & 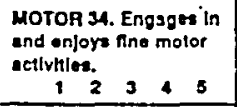 & 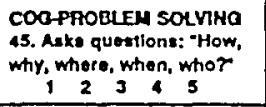 & $\begin{array}{l}\text { 54. Quenthes n.e ; counts } \\
\text { cars) } \\
12343\end{array}$ \\
\hline $\begin{array}{l}\text { 25. Forma and maintalns } \\
\text { friendehlpa. }\end{array}$ & 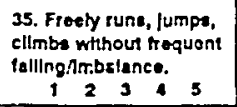 & 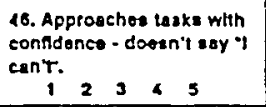 & 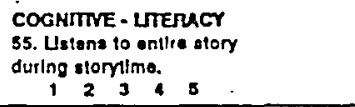 \\
\hline 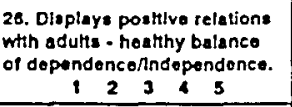 & 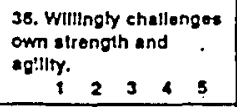 & 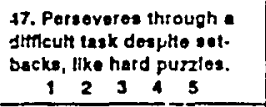 & 58. Aske to be reed to. \\
\hline 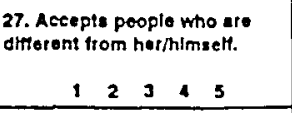 & 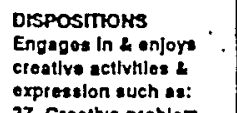 & $\begin{array}{l}\text { 80. Cont itlck with } \\
\text { activtles lor } 10 \text { minutes or } \\
\text { more. }\end{array}$ & 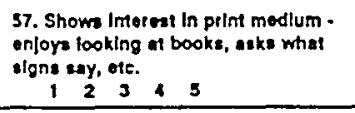 \\
\hline 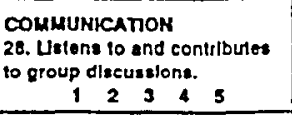 & 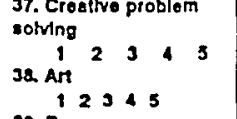 & 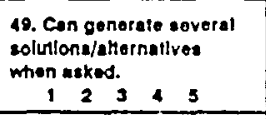 & 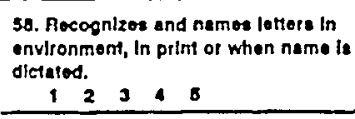 \\
\hline 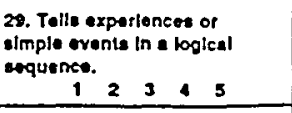 & 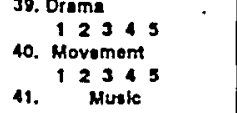 & 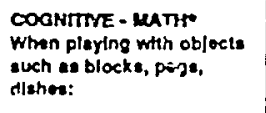 & 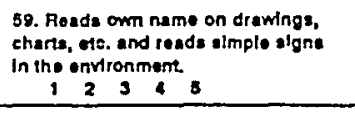 \\
\hline 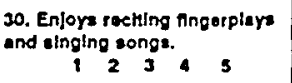 & 12345 & $\begin{array}{l}\text { 30. Matches: (1.e., rod cara } \\
\text { Irito ood comalneti) }\end{array}$ & 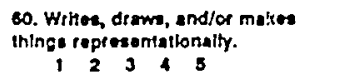 \\
\hline
\end{tabular}


Oregon Asesesement for 35 Y Yeat Olde In Developmentally Appropriate Clsestoome

\begin{tabular}{|c|c|c|c|c|}
\hline & Blrahdato: & Program: & Teachars: & \\
\hline 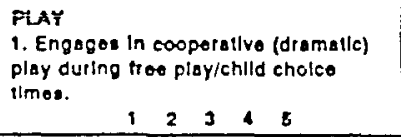 & 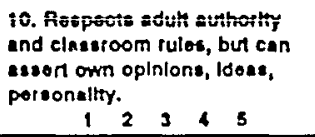 & 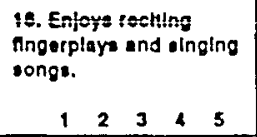 & $\begin{array}{l}\text { 26.Dlepleys } \\
\text { curlinsty. }\end{array}$ & $\begin{array}{l}\text { 34, Show Insorest in print modlum . } \\
\text { onjoys looking at books, eaks what } \\
\text { olgne say, otc. } \\
\begin{array}{lllll}1 & 2 & 3 & 4\end{array}\end{array}$ \\
\hline 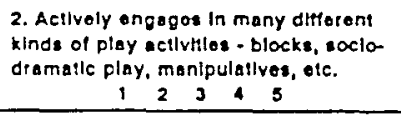 & $\begin{array}{l}\text { socul } \\
\text { 11. Hogotlatoe ottoctlvely } \\
\text { with othors. } \\
\begin{array}{rllll}1 & 2 & 3 & 4 & 5\end{array}\end{array}$ & $\begin{array}{l}\text { 19. Communleate so } \\
\text { o/he li understood by } \\
\text { others. } \\
123 \quad 4 \quad 5\end{array}$ & 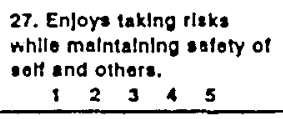 & 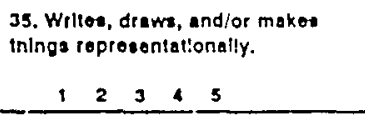 \\
\hline 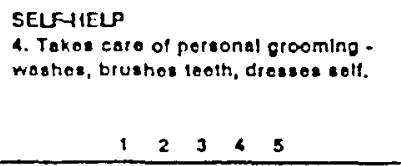 & 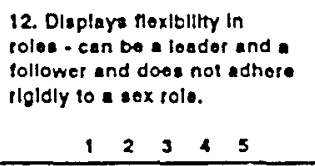 & 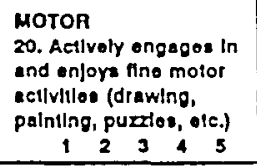 & 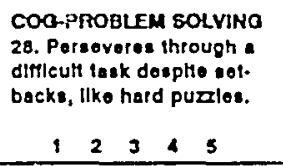 & COMMENTS: \\
\hline $\begin{array}{l}\text { 5. Wants lo help her/himiolf and } \\
\text { takes prido in dolng so. } \\
\qquad \begin{array}{lllll}1 & 2 & 3 & 4\end{array}\end{array}$ & $\begin{array}{l}\text { 13. Forms and maintalins } \\
\text { trlendehlps. } \\
\qquad \begin{array}{llll}1 & 2 & 3 & 5\end{array}\end{array}$ & $\begin{array}{l}\text { 21. Freety runs, jumps, } \\
\text { cllmbe whout frequent } \\
\text { falling or Imbalance. } \\
1\end{array}$ & $\begin{array}{l}\text { 29. Chooses ectlvilies and } \\
\text { can stlck with them for } 10 \\
\text { minutes or more. } \\
123\end{array}$ & \\
\hline 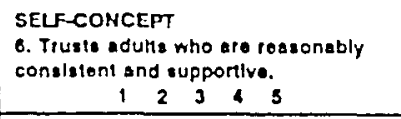 & 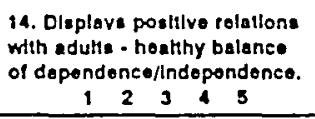 & 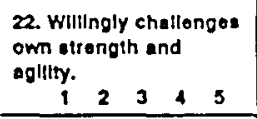 & $\begin{array}{l}\text { 30. Can generate sovoral } \\
\text { solutloni/athernatlves } \\
\text { whon aukod. } \\
\begin{array}{cccc}1 & 2 & 3 & 5\end{array}\end{array}$ & \\
\hline $\begin{array}{l}\text { 7. Requeals holp from aduls and } \\
\text { peors vertelly, polthely, and whien } \\
\text { sctually nooded. } \\
\qquad \begin{array}{llllll}1 & 2 & 3 & 4 & 5\end{array}\end{array}$ & 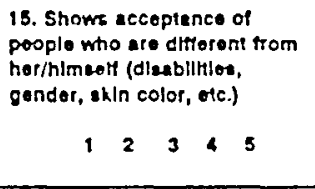 & $\begin{array}{l}\text { Drsposmons } \\
\text { Engages in } 2 \text { onjoyz } \\
\text { creatlve activiles it } \\
\text { expresslon sueh as: } \\
\text { 23. Art } \\
\begin{array}{ccccc}1 & 2 & 3 & 4 & 5\end{array}\end{array}$ & 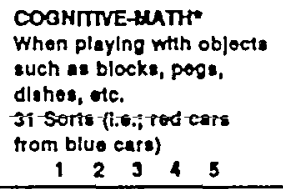 & \\
\hline 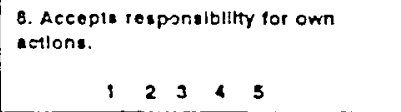 & 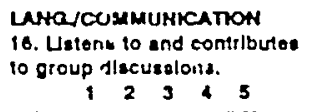 & $\begin{array}{l}\text { 24. Diame } \\
12345 \\
\text { 25. Movernom }\end{array}$ & $\begin{array}{l}\text { 32. Quaminos (1.e.i. counts } \\
\text { cantis) } \\
\begin{array}{lllll}1 & 2 & 3 & 4 & 5\end{array}\end{array}$ & \\
\hline $\begin{array}{l}\text { 9. Moves osally inrough tranetlons } \\
\text { touch ae circlo to enack). } \\
\qquad \begin{array}{llll}1 & 2 & 3\end{array} 4\end{array}$ & 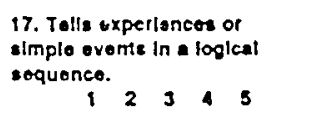 & & 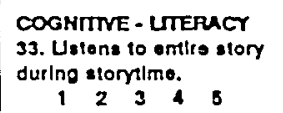 & \\
\hline
\end{tabular}

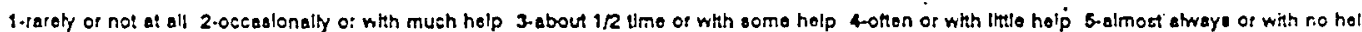

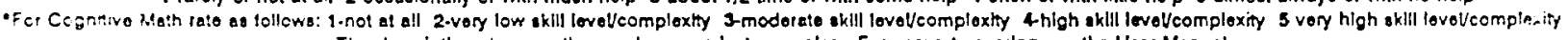
The descripllons in parentheses abovo are just examples. For eccurate acorlng seo the User Manuai 